Accelerator Division

AGS Department

\author{
BROOKHAVEN NATIONAL LABORATORY \\ Associated Universities, Inc. \\ Upton, NY 11973
}

AGS/AD/Tech. Note No. 406

\title{
Proposal on Spin Physics Using the RHIC Polarized Collider
}

14 August 1992

Abstract

We propose to study the spin dependence of various reactions using longitudinally and transversely-polarized beams at RHIC.

We present the design and construction of polarized beams, along with proposed measurements using PHENIX and STAR detectors. 


\section{Contents}

GENERAL INTRODUCTION ...........................................................................vii

\section{Section A}

\section{RHIC Polarized Collider and Experiments on Spin Physics}

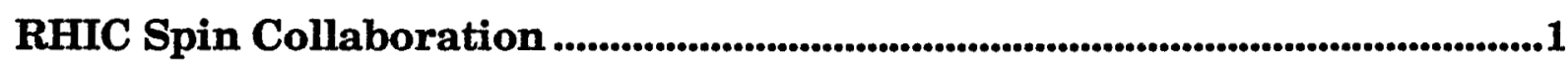

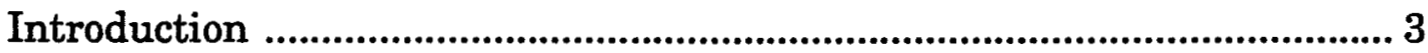

A.1 Physics Issues for the Polarized Collider...................................................... 6

A.1.1 Measurements of Parton Helicity Distributions in a Polarized Proton ...... 8

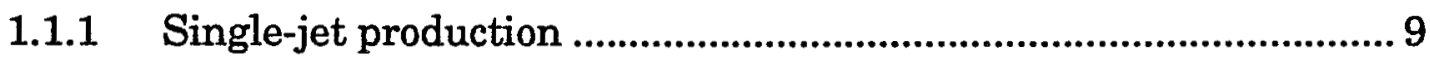

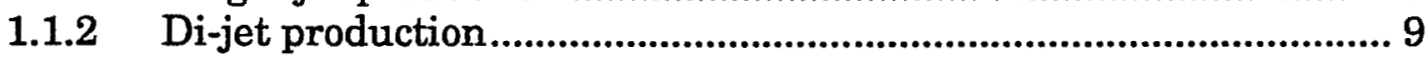

1.1.3 Direct-gamma production .........................................................10

1.1.4 Direct-gamma production with away-side jet detection................10

1.1.5 Drell-Yan ......................................................................................10

A.1.2 Measurements of the Quark Transversity Distribution in a Polarized Proton

1.2.1 Two-spin asymmetries .............................................................11

1.2.2 One-spin asymmetries ...............................................................12

A.1.3 Single Helicity Asymmetry - Parity Violation............................................13

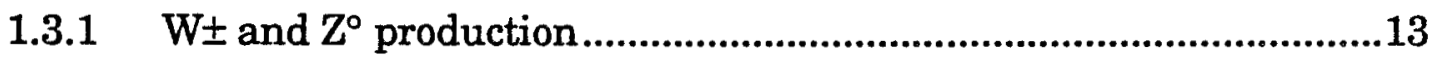

1.3.2 Compositeness ....................................................................................14

A.1.4 J/ $\Psi$ and Upsilon Production .....................................................................14

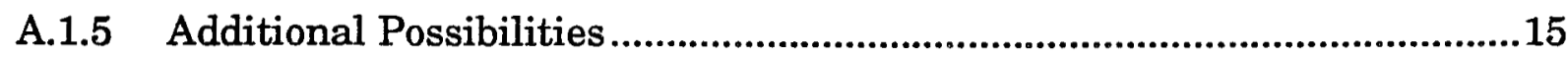




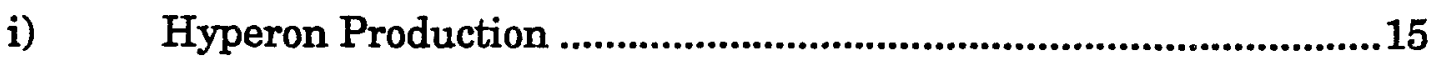

ii) Measurements of Spin Dependent Elastic Scattering and Total Cross Sections ...............................................................................15

A.2 Acceleration of Polarized Protons ......................................................... 16

A.2.1 Polarized Ion Source, AGS and Transfer of the Polarized Beams to RHIC

A.2.2 Siberian Snakes and Spin Rotators in RHIC .............................................17

A.2.3 Luminosity of Polarized Proton Collisions ...................................................19

A.2.4 Spin Reversal of the Stored Beams ..........................................................20

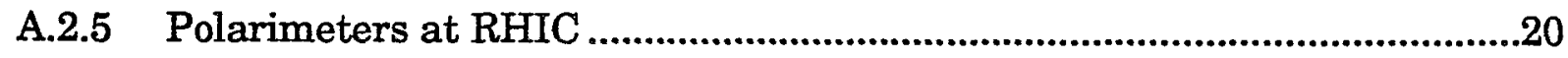

2.5.1 Coulomb-nuclear inteference ........................................................22

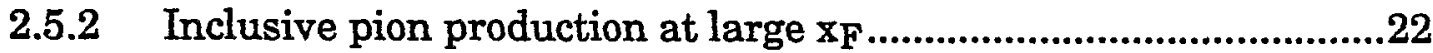

A.3 Conceptual Design Values and Cost Estimates for RHIC Spin Rotators/Siberian Snakes

A.4 Schedule and Request ................................................................................. 24

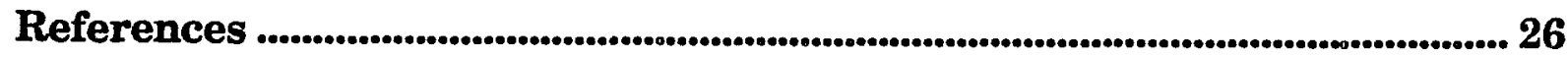

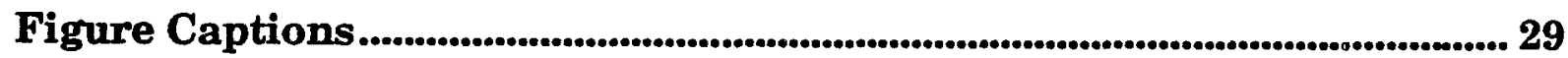




\section{Section B}

\section{Experiments with the PHENIX Detector}

PHENIX/SPIN Collaboration.......................................................................................1

B.1 Physics Goals and Capabilities .................................................................. 4

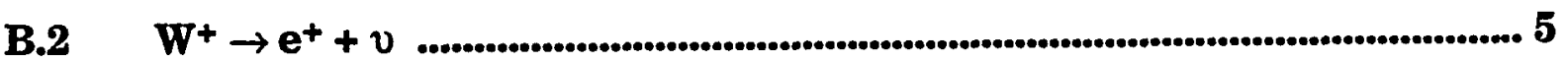

B.3 Direct Photon Production ..................................................................... 6

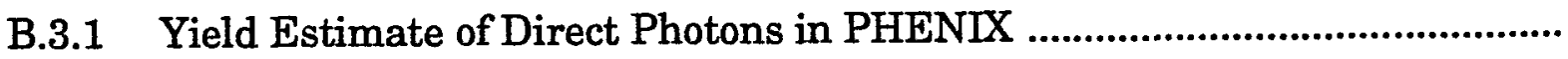

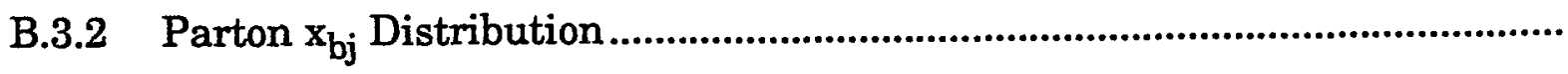

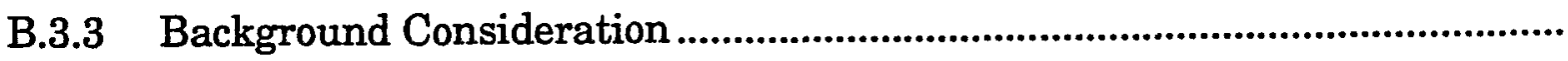

B.4 $\pi^{\circ}$ Production ............................................................................................

B.5. Other Physics Issues ….......................................................................... 11

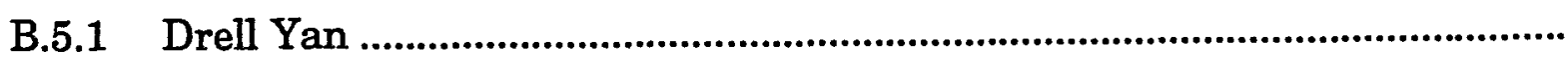

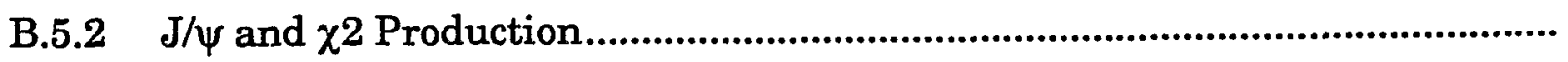

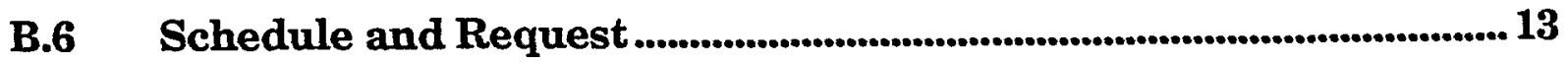




\section{Section C}

\section{Experiments with the STAR Detector}

STAR/SPIN Collaboration .....................................................................................................1

C.1 Physies Goals and Capabilities ..........................................................................3

C.2 Measurements with Barrel EMC and Shower Maximum Detector 5

C.2.1 Measurements of Parton Helicity Distributions in a Polarized Proton ...... 6

2.1.1 Direct-gamma production ..............................................................6

2.1.2 Single high-pT particle production ......................................................9

2.1.3 Single-jet production ........................................................................ 9

2.1.4 Direct-gamma + jet .......................................................................10

2.1.5 Di-jet production ..........................................................................

C.2.2 Measurements of the Quark Transversity Distribution in a

Polarized Proton .......................................................................................11

2.2.1 $A_{N}$ measurement in direct-gamma and $\pi^{\circ}$ production .................. 11

C.2.3 Single-Helicity Asymmetry in W and Z Production ..................................12

C.3 Measurements with Endcaps ..............................................................12

C.3.1 Measurements of $\Delta \mathrm{G}(\mathrm{x})$, Gluon Spin Structure Function.........................13

3.1.1 Detecting the Direct- $\gamma$ and the "Away-Side" Jet...........................13

3.1.2 Di-jet Production .....................................................................................14

C.3.2 Measurements of the Parton Helicity Distribution in the Drell-Yan Process .14

C.3.3 Measurements of $\mathrm{h}_{1}(\mathrm{x})$ in the Drell-Yan Process ........................................16 
C.3.4 W and Z Production

C4. Cost Estimates for STAR Upgrades 17

C5. Schedules and Requests 17

References 18 Figure Captions. 20 


\section{General Introduction}

We propose to collide beams of polarized protons in RHIC to:

-- $\quad$ Measure for the first time parity violation of real $\mathrm{W}$ and $\mathrm{Z}$ production in hadronic interactions;

-- Measure the gluon and sea quark polarization in longitudinally-polarized protons;

-- Measure the hard scattering parton-parton subprocess asymmetries and compare to perturbative QCD predictions for gg, gq, qq, and $\mathrm{q} \overline{\mathrm{q}}$;

-- $\quad$ Measure the quark polarization in transversely-polarized protons, both the double-spin transversity distribution, and the single spin measurement of correlations between quark fields and the gluon field strength.

This program requires special strings of dipole magnets in RHIC (Siberian Snakes), as well as polarimeters, and the two large heavy ion detectors PHENIX and STAR. PHENIX requires no changes for this spin program and STAR requires an EM calorimeter and a shower maximum detector. We anticipate that when polarized protons are colliding in RHIC, both large detectors will be used to study spin phenomena.

This proposal consists of three parts. The first, authored by the RSC group, describes the general RHIC spin physics program and proposes the hardware additions necessary to accelerate and collide polarized protons in RHIC. The second part of the proposal is for the spin program of the PHENIX detector, and is authored by the PHENIX/SPIN group. The third part of the proposal is for the spin program of the STAR detector, and is authored by the STAR/SPIN group. 


\title{
Section A \\ RHIC Polarized Collider and \\ Experiments on Spin Physics
}

\author{
by
}

M. Beddo, D. Grosnick, D. Lopiano, D. Hill, H. Spinka, D. Underwood, A. Yokosawa

High Energy Physics Division, Argonne National Laboratory, Argonne, Illinois 60439

G. Bunce, A. Carroll, E. Courant, R. Fernow, Y. Y. Lee, D. Lowenstein, Y. Makdisi, L. Ratner, T. Roser, M. Tannenbaum Brookhaven National Laboratory, Upton, New York 11973

J. B. Carroll, T. Hallman, G. J. Igo

University of California, Los Angeles, California 90024

N. Törnqvist,

University of Helsinki, Finland

N. Akchurin, Y. Onel

University of Iowa, Iowa City, Iowa 52242

S. Y. Lee

Indiana University, Bloomington, Indiana 47405

S. Hiramatsu, Y. Mori, H. Sato

KEK, National Laboratory for High Energy Physicss, Ibaraki-ken 305, Japan

L. Madansky and R. Welsh

Johns Hopkins University, Baltimore, Maryland 21218

H. Enyo, K. Imai, A. Masaike

Kyoto University, Kyoto, Japan

J. Soffer

Centre de Physique Theorique, Marseille, France

R. Jaffe

Department of Physics, Massachusetts Institute of Technology, Cambridge, Massachusetts 02139

A. Bravar, M. Conte, A. Penzo, M. Pusterla, P. Schiavon Genova-Padova-Trieste University and INFN, Italy 


\title{
J. Collins, S. Heppelmann, G. Ladinsky, E. Minor, R. Robinett \\ Pennsylvania State University, University Park, Pennsylvania, 16802
}

Yu. I. Arestov, B. V. Chuiko, A. M. Davidenko, A. A. Derevschikov, O. A. Grachov, A. K. Likhoded, A. P. Meschanin, S. B. Nurushev, V. L. Rykov, A. G. Ufimtsev, A. N. Vasiliev Institute for High Energy Physics, Protvino, Russia

\author{
A. Y. Efremov \\ JINR, Dubna, Russia \\ M. Werlen \\ University of Lausanne, Switzerland
}




\section{Introduction}

The extremely successful program of quantum chromodynamics (QCD) and electroweak tests at the hadron colliders at CERN and Fermilab has provided a wealth of information on the Standard Model of particle physics. Tests involving jets (single and multi-jet events), direct photons, Drell-Yan and weak boson production, and heavy quark and quarkonium production have probed the structure of the QCD hard-scatterings, often beyond leading order, and have yielded additional information on the parton structure of the proton itself. Perturbative QCD (PQCD) has progressed towards becoming a "precision" theory based on the confrontation with high-statistics and high-energy collider data much as the electroweak theory has been pushed to new limits by precise LEP data.

One aspect of our understanding that has not benefited from such experiments at high-energy colliders, however, is the area of spin physics, both the spin structure of the proton itself and the spin-dependence of the fundamental interactions. Deep inelastic scattering has historically been one of the main sources of information on parton distributions and polarized lepton-nucleon scattering experiments (such as by the YALE-SLAC and EMC experiments) have indeed provided improved information on the spin-structure of the valence quark distributions. Direct measurements of the longitudinally-polarized gluon and sea quark distributions, as well as information on the transverse quark (or transversity) distributions, is not directly accessible in such experiments, thus leaving open many questions concerning the "spin content" of the proton. Moreover, such measurements do not yield information on the fundamental spin structure of QCD itself.

Polarized proton-proton collisions, which can 'address both issues, have previously been restricted to relatively low-energy (by collider standards), fixed-target experiments. So spin, although historically central to the development of particle physics, has occasionally been relegated to a "soft physics" compartment in the field, despite indisputably large polarization effects seen in many different experiments. At issue has been the interpretation -- whether a QCD analysis is possible or whether a complete understanding on non-perturbative effects is necessary.

Design and construction of polarized beams, as well as the spin effects with the polarized protons at RHIC, have been discussed in the literature. ${ }^{1-3}$ We will quote some of them in this proposal.

With the successful tests of the Siberian Snake concept 4 and many design studies, it now seems clear that a polarization option at a hadron collider, especially 
at RHIC, is now a definite possibility. ${ }^{5-8}$ A comprehensive program of experiments at RHIC would allow one to measure the spin-dependent parton distributions of the proton (both longitudinal and transverse) and so would help to resolve any remaining "spin-crisis." Furthermore, it would provide fundamental spin tests of QCD and the electroweak interactions not accessible to unpolarized colliders (or other machines), probing the helicity structure of the matrix elements of the fundamental hard-scattering processes. Finally, the high energy and luminosity of such a machine would guarantee that a PQCD approach would be applicable. It would also be sufficient to produce copiously high $\mathrm{p}_{\mathrm{T}}$ jets and weak bosons while at the same time ensuring that the contributing quark processes are in a region of documented high polarization. In these regards, a RHIC spin program will be unique.

Spin physics tests using polarized beams and targets at fixed-target energies involving direct photons and quarkonium production have been put forth as possible probes of the longitudinally-polarized gluon distributions. The relatively low energies involved at presently-envisaged, polarized fixed-target facilities put the proposed experiments near the limit of applicability of PQCD. In contrast, polarized proton-proton collisions at RHIC, at collider energies of $\sqrt{\mathrm{s}}=50-500 \mathrm{GeV}$ and with high luminosity, reaching $\mathcal{L}=2 \cdot 10^{32} \mathrm{~cm}^{-2} \mathrm{sec}^{-1}$, would provide a huge sample of large transverse momentum events to which $\mathrm{PQCD}$ would be just as applicable as at existing unpolarized machines. Furthermore, this can likely be achieved while providing about $70 \%$ polarization in each beam. Even with limited running time (we use $4 \cdot 10^{6} \mathrm{sec}$ ), this would yield $800 \mathrm{pb}^{-1}$ of data and so would allow for high statistics studies of QCD and electroweak phenomena ${ }^{8}$ and their spin-dependence at a previously unattained level.

There are many exciting physics issues that can be explored at a polarized RHIC. Some of these issues focused upon in this proposal include:

- $\quad$ RHIC reaches the energy domain where parity violation dominates. A very large parity violation signal is expected for $\mathrm{W}$ and $\mathrm{Z}$ production, which has not been measured before for real Ws and Zs. We will measure this effect which, given maximal parity violation for the subprocess, directly measures the $\mathrm{u}$-quark polarization in a longitudinally-polarized proton for $\mathrm{W}^{+}$and $\mathrm{d}$ quark polarization for $\mathrm{W}^{-}$- This should agree with the deep inelastic scattering results for $\triangle \mathrm{u} / \mathrm{u}$ from SLAC and CERN. The parity-violation asymmetry should be about $40 \%$ for $\mathrm{W}^{+}$. In addition, we will search in general for parity violation in hadronic interactions. 
- A number of different reactions are sensitive to gluon or sea-quark polarization in a longitudinally-polarized proton. These parton spin distributions are fundamental. Although the gluon and sea-quark polarizations have traditionally been expected to be small, this is not necessarily so. The gluon field may well be polarized and be responsible, for example, for large single-spin asymmetries that have been observed, and also for the small apparent quark contribution to the proton spin as measured by the EMC experiment. We will use direct photon and jet production to measure the gluon polarization and dimuon (dielectron) production to measure the sea polarization.

- Parton-parton helicity asymmetries ( $\hat{a}_{L L}$ ) are expected to be very large, approaching unity in some cases. Different reactions at RHIC measure different products of (parton a polarization) $x$ (parton $b$ polarization) $x$ (subprocess asymmetry). For example, jets at lower $\mathrm{pT}_{\mathrm{T}}\left(\mathrm{x}_{\mathrm{T}}<0.1\right)$ measure the product $\left(\Delta \mathrm{g}_{\mathrm{a}}\right) \times\left(\Delta \mathrm{g}_{\mathrm{b}}\right) \times\left(\hat{\mathrm{a}}_{\mathrm{LL}}(\mathrm{gg})\right)$. Direct photons measure $\left(\Delta \mathrm{g}_{\mathrm{a}}\right) \times\left(\Delta \mathrm{q}_{\mathrm{b}}\right) \times\left(\hat{\mathrm{a}}_{\mathrm{LL}}(\mathrm{gq})\right)$, and $(\Delta q)$ is measured from $W$ parity violation and deep inelastic scattering. By using different reactions and different kinematic regions, we will measure the parton asymmetries for $\mathrm{gg} \rightarrow \mathrm{gg}$, gq $\rightarrow \gamma \mathrm{g}$, etc.

- With transversely-polarized protons, we will study the transverse spin structure of the proton. A spin-spin measurement with dileptons will provide the transversity spin distribution in a proton, $h_{1}(x)$. This new spin structure function, equally fundamental as the well-known helicity distribution $\mathrm{g}_{1}(\mathrm{x})$, is particularly sensitive to the relativistic treatment. The one-spin transverse asymmetry for high-pT direct photon production (and other observables), providing a twist-3 parton distribution, is sensitive directly to correlations between quark fields and the gluon field. Very large spin effects have already been seen for inclusive pion asymmetries and for inclusive hyperon polarization, but at moderate values of $\mathrm{p}_{\mathrm{T}}$, where parton arguments may not be applicable. At RHIC, the proposed measurements will be clearly in the hardscattering domain.

A program of collider spin physics is a crucial step in the assessment of the possible importance and technical feasibility of polarization options at future supercolliders, such as the SSC and LHC. It is clearly necessary that the parton content of the proton, including its spin dependence, be understood. RHIC is the ideal machine for pursuing this program.

The reactions we will emphasize are $\mathrm{W}$ and $\mathrm{Z}$ production, jet production, direct photon production, and dilepton production. The RHIC large detectors pro- 
vide a complementary coverage of these reactions. PHENIX emphasizes lepton and gamma physics with excellent granularity and triggering capability. PHENIX will measure W's, direct photons, dimuons, $\pi^{\circ}$ production, and $\mathrm{J} / \psi$ production, and does not require any detector modification to do this. STAR, with an EM calorimeter and shower maximum detector, has excellent coverage in pseudorapidity and azimuth for charged particle tracking and EM energy. It will measure $W$ and $Z$, jets, $e^{+} e^{-}$ pairs, $\pi^{\circ}$ s, direct photons, and direct photon + jet. Although the STAR TPC detector is inherently slow, studies show that at high pp luminosity the reconstruction efficiency is similar to that for central gold-gold events. We expect that STAR will operate efficiently at full RHIC pp luminosity.

We describe below the physics goals of the RHIC spin program and general detector considerations, followed by our proposed additions to RHIC to achieve polarized proton collisions at $\sqrt{\mathrm{s}}=500 \mathrm{GeV}, \mathcal{L}=2 \times 10^{32} \mathrm{~cm}^{-2} \mathrm{sec}^{-1}$, polarization = $70 \%$ in each beam. The detailed physics programs for the two RHIC detectors PHENIX and STAR and their hardware requirements are given in separate chapters.

\section{A.1 Physics Issues for the Polarized Collider}

With the polarized collider we will observe collisions between partons that will be included in the hard scattering domain. We are interested both in measuring the degree of polarization of the partons in the proton using a "known" analyzer, and in measuring the spin dependence of parton-parton scattering using beams of polarized protons. These measurements address fundamental issues -- the spin content of the proton, both longitudinal and transverse; the expected strong spin-dependence of parton-parton scattering; and correlations between quark spin and the gluon field in the proton. In addition, we will measure the parity violation in the production of $\mathrm{W}$ and $\mathrm{Z}$ bosons. In each case, the asymmetry derives from a product of parton polarizations and the analyzing power of the parton subprocess.

A spin formalism has been developed ${ }^{2}$ that closely resembles the more familiar unpolarized parton scattering formalism. The hard scattering hadronic reaction

$$
a+b \rightarrow c+X
$$


which is described in terms of two to two parton subprocesses in the QCD parton model as shown in Fig. A1. The corresponding inclusive cross section, provided factorization holds, is given by

$$
d \sigma(a+b \rightarrow c+X)=\sum_{i j} \frac{1}{1+\delta_{i j}} \int d x_{a} d x_{b}\left[f_{i}^{(a)} f_{j}^{(b)}+(i \leftrightarrow j)\right] d \hat{\sigma}_{i j}
$$

The summation runs over all contributing parton configurations; the parton distribution $\mathrm{f}_{\mathrm{i}}^{(\mathrm{a})}\left(\mathrm{x}_{\mathrm{a}}, \mathrm{Q}^{2}\right)$ is the probability that hadron "a" contains a parton " $\mathrm{i}$ " carrying a fraction $x_{a}$ of the hadron's momentum. The spin formalism replaces the spin-averaged fragmentation functions with helicity identification, $f \rightarrow f_{+}$or $f$, and the subprocess cross section now depends on the parton helicities, $\hat{\sigma}_{i j} \rightarrow \hat{\sigma}_{i+j}$, etc. It is then convenient to define the parton helicity asymmetry as

$$
\Delta f=f_{+}-f_{-},
$$

and subprocess așymmetries as

$$
\hat{a}_{L L} \mathrm{ij} \times d \hat{\sigma}_{i j}=d \hat{\sigma}_{++}-d \hat{\sigma}_{+\cdot \cdot}
$$

The value of these asymmetries are shown ${ }^{2}$ in Fig. A2 for different parton subprocesses. The double helicity asymmetry, ALL is given by

$$
A_{L L}=\frac{d \sigma_{++}-d \sigma_{+-}}{d \sigma_{++}+d \sigma_{+-}}
$$

Then, following the parton formalism, we have

$$
A_{L L} d \sigma=\sum_{i j} \frac{1}{1+\delta_{i j}} \int d x_{a} d x_{b}\left[\Delta f_{i}^{(a)} \Delta f_{j}^{(b)} \hat{a}_{L L}{ }^{i j} d \hat{\sigma}_{i j}+(i \leftrightarrow j)\right]
$$

We can also define two parity violation asymmetries as

$$
A_{L}=\left(d \sigma_{a+}-d \sigma_{-}\right) /\left(d \sigma_{a+}+d \sigma_{-}\right)
$$

and

$$
A_{L L} P V=\left(d \sigma_{++}-d \sigma_{-}\right) /\left(d \sigma_{++}+d \sigma_{-}\right)
$$




$$
=A_{L} \times\left[1+\left(d \sigma_{+-}+d \sigma_{-+}\right) /\left(d \sigma_{++}+d \sigma_{-}\right)\right],
$$

and similarly the transverse asymmetries $A_{N N}$ and $A_{N}$.

Our approach to physics at the parton level will be to choose reactions and kinematics where one partonic process is isolated, and where we have good sensitivity to the parton level measurement. We have used the Pythia simulation program to study the expected contributions of subprocesses to reactions and to choose favorable kinematics where one subprocess is dominant.

Typically, parton polarizations will be larger away from $x=0$, where $x$ is the parton momentum fraction. For valence quarks, their polarization could be large at large $x$. This was observed in deep inelastic scattering, as shown in Fig. A3. The quantity $A_{1}(x)$ basically measures the $u$ quark polarization $\Delta u / u$ in the proton.

From the figure for $x>0.2$, the $u$-quark polarization is larger than $40 \%$. For gluons and sea quarks, the number of these partons diverges as $x \rightarrow 0$, which leads to low polarization even if the helicity asymmetry is large. Details concerning coverage of $x$ values in measuring gluon and sea-quark polarization are described in C.2.1.4, C.3.1.1, and C.3.2.

To determine the gluon spin structure function, measurements in high-p direct photon production, where the Compton scattering of gluons and quarks dominates, are described in A.1.1.4 and C.3.1.1.

In the following sections we discuss the physics issues that will be addressed at RHIC.

\section{A.1.1 Measurements of Parton Helicity Distribution in a Polarized Proton}

Fundamental interactions at short distances, which are probed in pp collisions at high energies, involve hard scattering of quarks and gluons. In the hadronic reaction, $\mathrm{pp} \rightarrow$ (hadron or gauge boson) $+\mathrm{X}$, where both initial protons are longitudinally polarized, we measure an observable ALL (double helicity asymmetry) defined as

$$
A_{L L}=\left(1 / P^{2}\right)\left(N^{++}-N^{+-}\right) /\left(N^{++}+N^{+-}\right),
$$

that can be described in terms of the parton helicity asymmetry. ( $\mathrm{P}$ is the beam polarization and $\mathrm{N}^{++}\left(\mathrm{N}^{+-}\right)$are the number of events for parallel (antiparallel) spin states.) Results of the EMC/SLAC experiments suggest that very little of the proton spin is due to the helicity of the valence quarks inside it. The proton spin may re- 
ceive a large contribution from either the gluons, sea quarks, or angular momentum. However, quarks that carry a significant fraction of the proton momentum do have the same polarization as the proton.

At RHIC we can measure the gluon and sea-quark helicity distribution in the proton using jets, direct photons, and Drell-Yan pairs.

\section{A.1.1.1 Single-jet production (single-particle production)}

Several QCD subprocesses contribute to the cross section for jet production. The main contributions are gluon-gluon scattering, which dominate at low $\mathrm{p}_{\mathrm{T}}$, followed by gluon-quark scattering at medium pT (above $\sim 20 \mathrm{GeV} / \mathrm{c}$ ), and then quarkquark elastic scattering at high pT. These contributions are illustrated in Fig. C12.

By studying the asymmetry $A_{L L}$ in single-jet production, the effect from a large gluon polarization can be detected. ALL is proportional in terms of several QCD subprocesses as well as the gluon polarization $\Delta \mathrm{G} / \mathrm{G}$.

$$
\Delta G(x) / G(x)=\left[G^{\uparrow}(x)-G^{\downarrow}(x)\right] /\left[G^{\uparrow}(x)+G^{\downarrow}(x)\right],
$$

where $\Delta \mathrm{G}(\mathrm{x})$ is the longitudinal spin of the proton carried by gluon fields. The results of the calculations for a jet produced at rapidity $y=0$ are shown in Fig. A4 for a standard $\Delta G$ (Ref. 2) and a large positive $\Delta G$ (Ref. 9). Since for all the dominant subprocesses the corresponding asymmetries $\hat{a}_{L L}$ are positive, we expect a positive ALL.

For the pT region where gluon-gluon scattering is dominant,

$$
A_{L L}=\left(\Delta G\left(x_{a}\right) / G\left(x_{a}\right)\right) \cdot\left(\Delta G\left(x_{b}\right) / G\left(x_{b}\right)\right) \cdot \hat{a}_{L L}(g g \rightarrow g g) .
$$

The $\hat{a}_{\mathrm{LL}}$ is expected to be large, $\hat{\mathrm{a}}_{\mathrm{LL}}=0.8$ at $\mathrm{y}=0$.

\section{A.1.1.2 Di-jet Production}

The accepted cross section for di-jet production is much smaller than in the case of single-jet production. A prediction of $A_{L L}$ versus jet pair mass is shown in Fig. A5. The advantage over the single-jet production is the kinematic constraint on the momentum fractions, $x_{i}$, of the two partons.

Studies were performed to see if the kinematic constraint of large $x$ for one parton and small $x$ for the other leads to events that are due largely to a $u$ quark at 
large $x$ probing gluons at small $x$. Both jets would be at rapidity $\sim \pm 1$ to \pm 1.5 . While helpful, this still is not as clean as direct $\gamma$ plus jet.

\section{A.1.1.3 Direct-yProduction}

Direct-gamma production at high $\mathrm{pT}$ is probably one of the cleanest reactions to study perturbative QCD. Direct photons are produced through the quarkantiquark annihilation subprocess and the quark-gluon Compton subprocess, $q g \rightarrow \gamma q$. The Compton process is the dominant one in pp interactions, since there are no valence antiquarks in the proton. Then, the asymmetry $A_{L L}$ in direct- $\gamma$ production becomes directly proportional to $\Delta G(x)$. Direct photon events, without looking at the away-side jet, provide information about the integrals of $\Delta G(x)$ in certain ranges of $x$. The calculation of $A_{L L}$ with a large $\Delta G$ versus $p_{T}$ for different values of the c.m. production angles is shown in Fig. A6.

\section{A.1.1.4 Direct-Gamma Production with Away-Side Jet Detection}

In order to measure $\mathrm{G}(\mathrm{x})$, both the direct- $\gamma$ and the away-side jet must be detected in coincidence so that the kinematics of the incoming partons can be calculated. In the quark-gluon subprocess $q g \rightarrow \gamma q$, information about $\Delta G(x)$ can be obtained by measuring $A_{L L}(x)$ as:

$$
A_{L L}(x)=[\Delta u(x) / u(x)] \cdot[\Delta G(x) / G(x)] \cdot \hat{a}_{L L}(x),
$$

where $\left.\Delta u(x) / u(x)=\left[u^{\uparrow}(x)-u^{\downarrow}(x)\right] / u^{\uparrow}(x)+u^{\downarrow}(x)\right]$ with $\Delta u(x)$ being the longitudinal spin of the quark. The values of $\Delta u(x) / u(x)$ are obtained from results of the EMCSMC experiments and $\hat{a}_{\mathrm{LL}}$ are from theoretical calculations, yielding a value of 0.6 at $y=0$. The momentum fraction $x_{q}$ of the quark should be larger than 0.2 to 0.3 so that $|\Delta u(x) / u(x)|$ would be sizable. At $x_{q}=0.2$, we have $\Delta u(x) /(u) x=0.4$, and then the value $A_{L L}=0.2 \cdot \Delta G / G$ and $\delta(\Delta G / G)=5 \cdot \delta A_{L L}$.

\section{A.1.1.5 Drell-Yan}

The Drell-Yan process appears to be the only way to determine the polarization of sea quarks. The $q \bar{q}$ annihilation into a vector boson gives a large asymmetry on a partonic level, and selects sea antiquarks along with valence quarks. The polarization of valence quarks is known and the partonic-level asymmetry is known. 
It is possible from the measurement of the double spin asymmetry ALL to measure the sea-quark polarization. The measurement was discussed earlier ${ }^{10}$ in a kinematic region that is not easily accessible. The longitudinal spin-spin asymmetry ALL for Drell-Yan in pp collisions is related to the sea-quark helicity distrịbution:

$$
A_{L L}=\hat{a}_{L L} \frac{\sum_{i} e_{i}^{2}\left[\Delta \mathrm{q}_{i}\left(\mathrm{x}_{1}\right) \Delta \overline{\mathrm{q}}_{i}\left(\mathrm{x}_{2}\right)+\Delta \mathrm{q}_{i}\left(\mathrm{x}_{2}\right) \Delta \overline{\mathrm{q}}_{i}\left(\mathrm{x}_{1}\right)\right]}{\sum_{i} \mathrm{e}_{i}^{2}\left[\mathrm{q}_{i}\left(\mathrm{x}_{1}\right) \overline{\mathrm{q}}_{i}\left(\mathrm{x}_{2}\right)+\mathrm{q}_{i}\left(\mathrm{x}_{2}\right) \overline{\mathrm{q}}_{i}\left(\mathrm{x}_{2}\right)\right]},
$$

where $x_{1}-x_{2}=x F, x_{1} x_{2}=M^{2} / s$, and $\hat{a}_{L L}=-1$.

One can obtain an estimate of this quantity from a similar assumption made for the sea-quark polarization in Ref. 11, which will be discussed below. (See Fig. A7, up to the change of sign, because $\hat{a}_{N N}$ is positive and $\hat{a}_{L L}$ is negative.)

\section{A.1.2 Measurements of Quark Transversity Distributions in a Polarized Proton}

Recently, major theoretical progress was made in understanding and describing transverse spin effects in inclusive hard processes. Contributions from both dominant twist-2 and subdominant twist-3 processes were considered.11-19

\section{A.1.2.1 Two-spin Asymmetries}

From deep inelastic scattering, one can measure $f_{1}(x)$ related to the spinaveraged longitudinal momentum distribution of quarks in the nucleon and $g_{1}(x)$ related to the helicity distribution in a longitudinally-polarized nucleon. In addition to these two well-known structure functions, there exists a third fundamental function, $h_{1}(x)$, which is a leading-twist (twist-2) distribution function like $f_{1}(x)$ and $\mathrm{g}_{1}(\mathrm{x})$. This third function, which has never been experimentally determined, is accessible by measuring the double transverse spin asymmetry $A_{N N}$ in certain processes with both proton beams transversely polarized. The determination of $h_{1}(x)$ will allow one to predict $A_{N N}$ in various hard processes.

$A_{N N}$ is defined as,

$$
A_{\mathrm{NN}}=\left(1 / \mathrm{P}^{2}\right)\left(\mathrm{N}^{++}-\mathrm{N}^{+-}\right) /\left(\mathrm{N}^{++}+\mathrm{N}^{+-}\right)
$$


In terms of $h_{1}(x), A_{N N}$ is given ${ }^{11}$ as,

$$
A_{N N}=\hat{a}_{N N} \frac{\sum_{a} e_{a}^{2} h_{1}^{a}\left(x_{1}\right) h_{1}^{\bar{a}}\left(x_{2}\right)}{\sum_{a} e_{a}^{2} f_{1}^{a}\left(x_{1}\right) f_{1}^{\bar{a}}\left(x_{2}\right)},
$$

where $\hat{\mathrm{a}}_{\mathrm{NN}}$ is the parton's double-spin asymmetry. To obtain a large $\hat{\mathrm{a}}_{\mathrm{NN}}$ value, one can focus at the vicinity of $\theta_{\text {c.m. }}=\pi / 2$ and $\phi_{c . m .}=0$, where $\hat{a}_{\mathrm{NN}}=1$. One can then integrate over $\theta_{\text {c.m. }}$ and $\phi_{c . m}$. weighted with the sign of the asymmetry, 20 and this yields $\hat{a}_{\mathrm{NN}}=1 / \pi$ to obtain better statistics.

A measurement of $h_{1}(x)$ can shed interesting light on the spin structure of the nucleon. In fact, in non-relativistic quark models, the transversity distribution is identical to the quark helicity distribution $\mathrm{g}_{1}(\mathrm{x})$. Thus, a coinparison between the sizes of $h_{1}(x)$ and $g_{1}(x)$ would measure the success of these models in treating the spin degrees of freedom. As an example, Fig. A8 shows the results from the bag model for $g_{1}(x)$ and $h_{1}(x){ }^{21}$ Using this result, one can calculate the double transverse spin asymmetry $A_{N N}$ for the Drell-Yan process with transversely-polarized beams. These results are shown in Fig. A7.11

Another observation about $h_{1}(x)$ is that its unweighted sum rule is chargeconjugation odd, and thus only valence quarks contribute to it. This leads to the conclusion that this sum rule has a much better chance to be predicted in valence models than the $g_{1}(x)$ sum rule.

\section{A.1.2.2 One-spin Asymmetries}

Single transverse spin asymmetries in hard processes are expected to vanish in perturbative $\mathrm{QCD}$. The asymmetries vanish at the leading-twist (twist-2) level, however this is no longer true if one considers higher-twist contributions. These higher-twist techniques were applied to the simple case of $\mathrm{pp} \rightarrow \gamma \mathrm{X}$, in which the twist-3 contribution, leading to a potentially larger asymmetry, increases with $\mathrm{XF}{ }^{18-19}$ The twist-3 parton distribution involves the correlation between quark fields and the gluon field strength. Clearly the normalization, sign, and $x-$ dependence of this new partonic distribution will remain unknown until it is measured.

A measurement of higher-twist effects not only provides a valuable test on perturbative QCD, it also yields information on the hadron structure. The higher- 
twist distributions represent correlations between quarks and gluons inside a $\therefore . i$ hadron. These correlations have specific structures in QCD. If extracted from ' $1 \mathrm{~A}(\mathrm{X} ;$ experimental data, they provide useful constraints on nucleon models, in particulär, the non-valence degrees of freedom that include sea quarks and gluons.

$-\because \mathrm{i}: \mathrm{i}$ is oe

\section{A.1.3 Single Helicity Asymmetry - Parity Violation}

The observable $\mathrm{A}_{\mathrm{L}}^{\mathrm{PV}}$ is defined as,

$$
A_{L}^{P V}=\left(N^{-}-N^{+}\right) /\left(N^{-}+N^{+}\right)
$$

where $-(+)$ are minus (plus) helicity, respectively.

$\mathrm{A}_{\mathrm{L}}^{\mathrm{PV}}$ is expected to vanish unless some partonic subprocesses involve the parity-violating interaction. At nominal energy, all the parity-violating asymmetry would come from the electroweak interaction, however it may be enhanced by the to: proton structure.

When the helicities of both beams are the same, we define another parity- $\therefore-1$ violating asymmetry, namely,

$$
A_{L L}^{P V}=\left(N^{--}-N^{++}\right) /\left(N^{--}+N^{++}\right)
$$

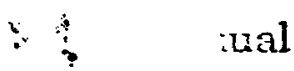$$
\therefore \cdot
$$

which can be about twice as big as $\mathrm{A}_{\mathrm{L}}^{\mathrm{PV}}$ for special cases,

\section{A.1.3.1 $W \pm$ and $Z^{\circ}$ Production}

$\mathrm{W}$ bosons are predicted to be produced by a parity-violating mechanism, $: \mathrm{C}_{i}$ using Standard Model couplings. The asymmetry $\mathrm{A}_{\mathrm{L}}^{\mathrm{PV}}$, or PVA, is predicted to be ${ }^{8} \mathrm{a}$ about $35 \%$ in $\mathrm{W}$ production, 2,22 and the PVA can be measured in the hadtoproduc $\mathrm{c}$ : tion of $\mathrm{W} \rightarrow \mathrm{e}+v, \mu+v$. This remarkable effect will be seen for the finst time at $\mathrm{v}$ RHIC.

The asymmetry $\mathrm{A}_{\mathrm{L}}^{\mathrm{PV}}$ for $\mathrm{W}^{+}$production is proportional to the u-quark polarization at large $\mathrm{x}$ in the proton. By using the deep inelastic scattering result for $\Delta \mathrm{u} / \mathrm{u}$ and the expected result for $\Delta \mathrm{d} / \mathrm{d}$, a value of $\mathrm{A}_{\mathrm{L}}^{\mathrm{PV}}$ will give a direct measure of parity violation at the parton level. These results will also serve as a self-consistency check of the experimental technique. For example, do these results and the deep inelastic scattering results measure the same $u$ and $d$ quark pol 
- Figure A9 shows the single helicity asymmetries, $A_{L}(y)$ versus rapidity $y$ for $\mathrm{W}^{+}$and $\mathrm{W}^{-}$production. ${ }^{23}$ For $\mathrm{W}^{+}, \mathrm{A}_{\mathrm{L}}^{\mathrm{PV}}$ has the trend of $\Delta \mathrm{u}(\mathrm{x}) / \mathrm{u}(\mathrm{x})$, and for $\mathrm{W}^{-}$it has the trend of $\Delta d(x) / d(x)$. Figure $A 10$ shows prediction for $A_{L}^{P V}(y)$ for $Z^{\circ}$ production. ${ }^{23}$

Non-leptonic $W$ decays can be isolated through a parity-violating subtraction. As an example, the process of $\mathrm{W} \rightarrow 2$ jets can be studied. ${ }^{2}$

\section{A.1.3.2 Compositeness}

Quarks and leptons may consist of more fundamental constituents, and have a composite structure. The fascinating feature of composite models of quarks and leptons is that the interactions generally violate parity. By measuring PVA, the composite structure may be studied.

\section{A.1.4 J/Y and Upsilon Production}

Hadroproduction of heavy quarkonia has a long history, beginning with the discoveries of both the $J / \psi$ and $\Upsilon$. It is now well established that gluon fusion is the dominant production mechanism for these processes in high-energy pp collisions and data on $\psi / \Upsilon$ production were one of the first sources of direct information on the unpolarized gluon distributions. ${ }^{24}$ Analyses using "local duality" ideas 25 successfully explained a large body of data on inclusive $\psi$ and $\Upsilon$ production while more extensive calculations, based on potential models, ${ }^{26}$ can also predict more detailed properties (such as $\sigma(\chi \rightarrow \psi \gamma) / \sigma(\psi)$ ). Such models successfully predicted collider data on low $\mathrm{p}_{\mathrm{T}} \Upsilon$ and high $\mathrm{p}_{\mathrm{T}} \psi$ production 27 and are also used to analyze electro- and photoproduction experiments.

The spin-dependence of heavy quarkonium production can also be predicted in such models and $\chi_{2}(\mathrm{c} \overline{\mathrm{c}})$ production has been suggested ${ }^{28}$ as a direct measure of the polarized gluon content of the proton (assuming P-states can be suitably reconstructed from their $\psi+\gamma$ decays). If $\chi$ states cannot be resolved, then inclusive $\psi$ production (with other contributing mechanisms such as gg $\rightarrow \mathrm{g} \psi$ ) may also be studied since it has a significant spin dependence as well. ${ }^{29}$ The spin dependence of the

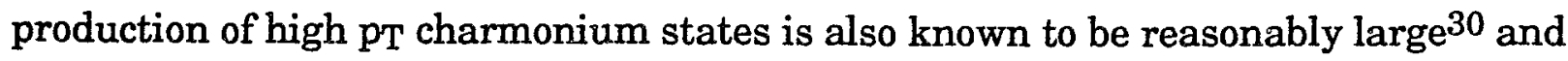
might also be measured.

$\Psi$ production has been cited as being of interest in the context of heavy ion collisions as well (for example, as a possible diagnostic for the production of quarkgluon plasma) so that there may well be a strong physics overlap between the heavy ion and spin physics programs for this case. 


\section{A.1.5 Additional Possibilities}

i) Hyperon Production

Here we consider the production of hyperons in the kinematic region where $\mathrm{p}_{\mathrm{T}}$ is small, say $\mathrm{p}_{\mathrm{T}} \sim 1 \mathrm{GeV} / \mathrm{c}$, and $\mathrm{x}$ is around 0.5 . In this case for $\Lambda$ production, a rather simple mechanism has been proposed 31 that is consistent with scaling (no dependence on energy), and explains the observed $\Lambda$ polarization in pp collisions using unpolarized protons rather well. It would be interesting to check if this scaling property holds far above ISR energies in the RHIC energy domain, and which features of the other observables ( $A, D_{N N}, A_{L L}$, etc.) can be measured with one and two polarized beams. The Fermilab experiment seems to be less energy dependent for the production of $\Sigma^{+}$and also they have definitely observed that for $\Xi^{-}$the polarization at $800 \mathrm{GeV} / \mathrm{c}$ is larger than at $400 \mathrm{GeV} / \mathrm{c}$. A systematic study of all spin observables for the different hyperon productions could be done at RHIC in an optimal way.

ii) Measurements of Spin-Dependent Elastic Scattering and Total Cross Sections

At the RHIC energy region from $\sqrt{\mathrm{s}}=50 \mathrm{GeV}$ to $500 \mathrm{GeV}$, we can investigate the spin-dependent elastic scattering at small angles and total cross sections. We can obtain several observables, $A_{N}$ and $A_{i k}(i, k=N, S, L)$ by one-spin and twospin asymmetry measurements at relatively small $|t|$.

Measurements of $\Delta \sigma_{\mathrm{L}}$ (longitudinal) and $\Delta \sigma_{\mathrm{T}}$ (transverse), the difference of total cross sections in the initial pure spin states, are one of the most fundamental measurements needed in the study of forward hadron interactions. These quantities are defined as

$$
\begin{aligned}
\sigma^{\text {Tot }} & =(2 \pi / \mathbf{k}) \operatorname{Im}\left\{\phi_{1}(0)+\phi_{3}(0)\right\}, \\
\Delta \sigma_{\mathrm{L}} & =(4 \pi / \mathrm{k}) \operatorname{Im}\left\{\phi_{1}(0)-\phi_{3}(0)\right\}, \text { and } \\
\Delta \sigma_{\mathrm{T}} & =-(4 \pi / \mathrm{k}) \operatorname{Im} \phi_{2}(0),
\end{aligned}
$$

where $\phi_{1}, \phi_{2}, \phi_{3}$ are s-channel helicity amplitudes. These measurements will be useful to shed light into the growth of total cross section with energy. 


\section{A.2 Acceleration of Polarized Beams}

We describe the technical considerations relevant for producing, storing, and monitoring polarized proton beams, of the proposed spin RHIC complex (see Fig. A11).

\section{A.2.1 Polarized Ion Source. AGS Preparations, and Transfer of the Polarized Beam to RHIC}

To achieve high luminosity, high-energy polarized proton collisions in RHIC, the intensity of the present AGS polarized proton source is sufficient. The source ${ }^{32}$ produces $30 \mu \mathrm{A}$ of $\mathrm{H}^{-}$with $75-80 \%$ polarization in $300 \mu$ s pulses at a repetition rate of $5 \mathrm{~Hz}$. The polarized $\mathrm{H}$ - ions are accelerated to $200 \mathrm{MeV}$ with an RFQ and $200 \mathrm{MHz}$ LINAC. Twenty pulses of $\mathrm{H}^{-}$ions are strip-injected, accumulated, and captured into a single bunch in the AGS Booster with an estimated efficiency of about 50\%. Each bunch in the booster will then contain $\mathrm{N}_{B}=6 \times 10^{11}$ polarized protons with a normalized emittance of about $\varepsilon_{\mathrm{N}}=10 \pi \mathrm{mm}$-mrad depending on the harmonic number of the rf system used. The single bunch of polarized protons is accelerated in the Booster to $1.5 \mathrm{GeV}$ kinetic energy and then transferred to the AGS, where it is accelerated to $25 \mathrm{GeV}$.

During acceleration, the polarization may be lost when the spin precession frequency passes through a so-called depolarizing resonance. These resonances occur when the number of spin precession rotations per revolution $G \gamma(G=1.793$ is the anomalous magnetic moment of the proton, $\gamma=\mathrm{E} / \mathrm{m}$ ) is equal to an integer (imperfection resonances), or equal to $\mathrm{kP} \pm v_{\mathrm{y}}$ (intrinsic resonances). Here $\mathrm{P}=12$ is the super period of the AGS, $v_{\mathrm{y}}=8.8$ is the vertical betatron tune, and $\mathrm{k}$ is an integer. Imperfection resonances are due to vertical closed orbit errors and intrinsic resonances are due to the vertical betatron motion. The two resonances in the booster $\left(\mathrm{G}_{\gamma}=3\right.$ and $\left.\mathrm{G}_{\gamma}=4\right)$ are too weak to cause any significant depolarization. Traditionally, the depolarizing resonances in the AGS were corrected by the tedious harmonic correction method for the imperfection resonances and the tune jump method for the intrinsic resonances. 33

In the approved experiment (E-880) at the AGS to be started in 1993, the feasibility of polarized proton acceleration by using a 5\% solenoid Siberian Snake ${ }^{34}$ will be tested. Previous experience with polarized proton acceleration in the AGS indicates that a $5 \%$ snake is sufficient to avoid depolarization due to the imperfection 
resonances without using the harmonic correction method. The remaining six important intrinsic resonances can be corrected by the proven tune jump method. At $25 \mathrm{GeV}$, the polarized protons are transferred to RHIC. At this energy the transfer line between the AGS and RHIC is spin transparent. ${ }^{35}$

We estimate that the overall efficiency of the acceleration and beam transfers is better than $50 \%$, giving $2 \times 10^{11}$ proton/bunch, and the normalized emittance will increase to $20 \pi \mathrm{mm}$-mrad. We repeat the process until all 57 bunches of each ring are filled. Since each bunch is accelerated independently, we have the option of preparing the polarization direction of each bunch independently. Filling both RHIC rings with 57 bunches each and acceleration to full energy will take about 10 minutes and the lifetime of the stored polarized-proton beams in RHIC is many hours.

\section{A.2.2 Siberian Snakes and Spin Rotators in RHIC}

By inserting two full Siberian Snakes in each of the two RHIC rings, depolarization from depolarizing resonances can safely be avoided up to the top energy of $250 \mathrm{GeV}$, provided the normalized emittance is not larger than $20 \pi \mathrm{mm}$ mrad, the vertical RMS closed orbit is corrected to less than $0.3 \mathrm{~mm}$, and the low-order snake resonances ${ }^{36}$ are avoided. 6 The expected beam polarization is about $70 \%$.

Each snake rotates the spin by $180^{\circ}$ around a horizontal axis, and the two axes of the two snakes of each ring have to be perpendicular on each other, i.e. one snake rotates around the longitudinal axis and the other around the radial axis. Also, the two snakes of each ring have to be installed on opposite sides of the ring, and in fact, the beam direction in one snake has to be exactly opposite to the beam direction in the other snake to within $0.3 \mathrm{mrad}$. The only straight sections available for the installation of the Siberian Snakes are located in the insertions on either side of the interaction point. Unfortunately however, two of these straight sections that are on opposite sides of the ring are not parallel but span an angle of $7.34 \mathrm{mrad}$, much more than can be tolerated. Figure A12 shows the present design of one of the insertions and also a possible modification that would make the straight section parallel to the collision straight section and therefore parallel to the corresponding straight section on the opposite side of the ring. With such a modification the straight section would be suitable for the installation of the Siberian Snakes.

In addition to the Siberian Snakes, spin rotators are required at the intersection points used by PHENIX and STAR to allow for measurements of spin effects 
with either longitudinally- or transversely-polarized protons. Therefore, the spin rotators rotate the polarization from the vertical direction into the horizontal plane on one side of the interaction region and restore it to the vertical direction on the other side. In fact, such a pair of spin rotators can also be used to act as a Siberian Snake ${ }^{37}$ if the polarization direction of the outgoing beam is opposite to the one of the incoming beam.

The following proposal for the location and construction of the Siberian Snakes and the spin rotators (see Fig. A11) primarily aims at demonstrating the feasibility of satisfying all the requirements listed above:

- Two full Siberian Snakes, one in each ring, are installed at the 12 o'clock region with the insertion modification as shown in Fig. A12. These snakes rotate the spin around the radial axes (also called Type II Snakes).

- At the 6 o'clock region, the two pairs of spin rotators for STAR are configured to act as Siberian Snakes that rotate the spin around the longitudinal axis (also called Type I Snakes). This insertion would also have to be modified as shown in Fig. A12. A six-magnet spin rotator described in Ref. 38 is used that can be adjusted to provide either radial or longitudinal polarization at the interaction region while leaving alone the overall Snake of Type I. These spin rotators are used as snakes and cannot be turned off. It is therefore not possible to collide vertically-polarized beams at this intersection. Since the STAR detector is azimuthally symmetric, all transverse-spin measurements can be done with radial polarization.

- The two pairs of spin rotators for PHENIX at the 8 o'clock region are configured to restore the beam polarization after the intersection region. This means that in addition to longitudinal polarization it is also possible to turn the spin rotators off and collide vertically-polarized beams. Since the PHENIX detector only has coverage around the horizontal plane, vertical polarization is necessary to measure transverse spin effects.

- The orbit excursions inside the spin rotators are significant, particularly at low energy. For radial polarization at the collision point, the orbit excursion at $25 \mathrm{GeV}$ is about $70 \mathrm{~mm}$ both in the horizontal and vertical plane for the design that is being discussed later. When the spin rotators are configured 
for longitudinal polarization at the collision point, the excursions are three times this value in the horizontal plane. For this reason we are planning to always configure the spin rotators for radial polarization at injection and during acceleration, and only switch to longitudinal polarization at an energy of $75 \mathrm{GeV}$ or higher if needed by the experiments. The change of polarization direction can be done adiabatically without loss of polarization since the overall snake type remains unchanged for the spin rotators of Ref. 15.

\section{A.2.3 Luminosity of Polarized Proton Collisions}

The luminosity at the RHIC top energy of $250 \mathrm{GeV}$ is given ${ }^{7}$ by

$$
\mathcal{L}=5.7 \cdot 10^{31} \frac{\left(\frac{\mathrm{N}_{\mathrm{B}}}{10^{11}}\right)^{2}\left(\frac{\mathrm{B}}{57}\right)\left(\frac{\mathrm{E}}{250}\right)}{\left(\frac{\varepsilon_{\mathrm{N}}}{20 \pi}\right)^{2}} \mathrm{~cm}^{-2} \mathrm{sec}^{-1},
$$

where $B$ is the number of bunches in each ring, $N_{B}$ is the number of particles per bunch, and $\varepsilon_{\mathrm{N}}$ is the normalized emittance. Thus the polarized-proton luminosity will be $2 \times 10^{32} \mathrm{~cm}^{-2} \mathrm{~s}^{-1}$, which corresponds to two interactions per crossing for the $\mathrm{B}=57$ bunches per ring. Note the importance of smaller emittance in obtaining higher luminosity. The luminosity will gain a factor of four when the emittance is smaller by a factor of two. This is due to the fact that the betatron amplitude function $\beta^{*}$ at the interaction point can be squeezed by a factor of two at the smaller emittance while maintaining the same dynamic aperture at the high betatron amplitude quadrupole triplets. This is also the reason behind the linear dependence of the luminosity on energy, since the unnormalized emittance depends on $E^{-1 / 2}$.

We show in Fig. A13 the luminosity versus energy 39 as described above ("enhanced luminosity"), emphasizing the linear dependency of the luminosity on energy. The RHIC design luminosity for pp is also shown that, for the emittance used above, would represent $5 \times 10^{10}$ protons per bunch. 


\section{A.2.4 Spin Reversal of the Stored Beams}

Since the proposed asymmetry measurements are high-precision measurements, frequent polarization sign reversal is imperative to avoid systematic errors. Possible sources for systematic errors are luminosity variations, crossing angle variations, and detector efficiency variations. As mentioned earlier, different bunches will have different polarization sign and therefore different bunch crossings will measure interactions with different combinations of incoming beam polarization signs. Although this will greatly reduce systematic errors, it is still true that one pair of bunches would always cross with the same combination of polarization signs during the whole lifetime of the stored beams, which is at least several hours. To eliminate the possibility of systematic errors from this situation, we propose to install a spin flipper in each ring which is capable of reversing the polarization sign of all bunches. The spin flipper consists of three horizontal DC dipole magnets interleaved with three laminated vertical dipole magnets. Exciting the vertical magnets with about $40 \mathrm{kHz} \mathrm{AC}$ current would drive an artificial spin resonance that can be used to adiabatically reverse the polarization direction. Figure A14 shows the result of a test of this concept performed at the Indiana University Cyclotron Facility (IUCF). 40 We estimate that a complete spin reversal would take less than one second.

\section{A.2.5 Polarimeters at RHIC - CNI, Large- $x_{-} \pi$ 'S}

It is imperative that the beam polarization is known at every step in the acceleration cycle in order to assure that the beam is injected into RHIC with maximal polarization. Similarly, since the stored beam has an expected lifetime of about ten hours, the experiments need to know the average beam polarization during the store at intervals of much shorter duration.

Generally, transverse polarization is measured by the polarimeters, and the polarization direction throughout the acceleration cycle is vertical. Vertical polarization is determined by measuring the left-right asymmetry in the horizontal plane:

$$
P_{B}=1 / A\left[N_{L}-N_{R}\right] /\left[N_{L}+N_{R}\right]
$$


where $A$ is the analyzing power of the reaction and $N_{L}$ and $N_{R}$ are the scatters into the left and right polarimeter arms respectively.

At $200 \mathrm{MeV}$, the beam polarization is measured in proton-carbon scattering from a target filament at two scattering angles, 12 and 16 degrees, with analyzing powers of .62 and .51, respectively. A third polarimeter in the same setup measures the up-down asymmetry, which is expected to be zero. This provides a measure of the systematic errors and beam positioning. During previous polarized runs with the AGS, this provided an absolute measurement with high rates such that a $2 \%$ error was reached in a few minutes.

Also during the previous runs, the AGS used an internal polarimeter that measured pp elastic scattering into recoil arms set at about 77 degrees. The elastic scatters had momenta of several hundred $\mathrm{MeV} / \mathrm{c}$ as the circulating beam energy increased from $3 \mathrm{GeV}$ to $25 \mathrm{GeV}$. The polarimeter employed three sets of scintillating counters and aluminum absorbers in each arm. The counters suffered from a high rate of accidentals, this in addition to the background from the inclusive spectrum, reduced its effective analyzing power to approximately $40 \%$ of the pp elastic scattering analyzing power, which varied with energy. This system was used as a relative polarimeter. It should be noted that the measurements with the internal polarimeter are taken at a flat top in the acceleration cycle.

Both of these polarimeters will be upgraded as part of the approved AGS experiment E-880, which is scheduled to take data with a polarized beam by the summer of 1993.

We expect that the small-angle elastic scattering experiment (RLOI1) will make an absolute measurement of the beam polarization. Preliminary studies indicate that one can accomplish the measurement within several hours.

Polarimeters for the polarized beams at the ZGS and AGS up to $25 \mathrm{GeV} / \mathrm{c}$ relied on the analyzing power in pp elastic scattering at small $|t|$. For higher energies, such polarimeters can no longer be used because the analyzing power becomes very small at $|t| \leq 0.3(\mathrm{GeV} / \mathrm{c})^{2}$, and the cross section becomes small at $|t| \geq$ $1.5(\mathrm{GeV} / \mathrm{c})^{2}$ where the analyzing power is relatively large.

For the Fermilab polarized-beam facility at $200 \mathrm{GeV} / \mathrm{c}$, two kinds of polarimeters were developed and both of these can be used at energies much higher than $200 \mathrm{GeV} / \mathrm{c}$. These polarimeters were used with extracted beams only and presently no precise and concrete plan exists on their use in collider mode. Since the RHIC beam energies are similar to the present Fermilab polarized beams, and large 
asymmetries were found in $\pi^{\circ}, \pi^{+}$, and $\pi$ inclusive production at $200 \mathrm{GeV} / \mathrm{c}$, these can also be applied to design beam-polarization monitors.

\section{A.2.5.1 Coulomb-Nuclear Interference (CNI Polarimeter)}

Several authors since Schwinger 41 have indicated nonzero polarization in the Coulomb-nuclear interference region in nucleon-nucleon scattering. A polarimeter based on this measures the interference term of the nonflip amplitude and the electromagnetic spin-flip amplitude. The proton polarization arising from the interference is $\mathrm{P} \approx 5 \%$ at $|\mathrm{t}|=2 \cdot 10^{-3}(\mathrm{GeV} / \mathrm{c})^{2}$ and is energy independent. 42

The analyzing power, $A_{N}$, of proton-proton, proton-hydrocarbon, and antiproton-hydrocarbon scattering in the Coulomb-nuclear region was measured using the $185-\mathrm{GeV} / \mathrm{c}$ Fermilab polarized-proton and polarized-antiproton beams. 43 For the elastic scattering at small $|t|$, a set of scintillation counter targets was utilized to detect the recoil proton that stops within a very short range within the scintillator. At $|t| \sim 0.003(\mathrm{GeV} / \mathrm{c})^{2}$, a value of $A_{N}=(2.4 \pm 0.9) \%$ with the polarizedproton beam on a hydrocarbon target, and $\mathrm{A}_{\mathrm{N}}=(4.5 \pm 2.8) \%$ with proton-proton scattering. These results are consistent with predictions ${ }^{44-46}$ based on Coulombnuclear interference.

Recently, a new CNI target consisting of trans-stilbene crystals (diphenylethylene, $\mathrm{C}_{14} \mathrm{H}_{12}$ ) was used. 47 This made it possible to reduce the large background in the small- $|t|$ region by using this material that possesses pulseshape discrimination characteristics. Preliminary results on the pp analyzing power are shown in Fig. A15 together with the calculated values.

\section{A.2.5.2 Inclusive Pion Production at Large $x_{F}$}

Asymmetry measurements ( $A_{N}$ ) on the $x_{F}$ dependence of $\pi$ production (covering pT up to $2 \mathrm{GeV} / \mathrm{c}$ ) using polarized protons at $200 \mathrm{GeV} / \mathrm{c}$ were performed at Fermilab. 48 The $A_{N}$ values in the pp reaction are consistent with zero up to $x_{F}=$ 0.3 , and then increase linearly up to the absolute value of $40 \%$ near $\mathrm{xF}_{\mathrm{F}}=1.0$, as shown in Fig. A16. These effects can be applied to the design of beam polarimeters and beam-polarization monitors. 


\section{A3. Conceptual Design Values and Cost Estimates for RHIC Spin Rotators/Siberian Snakes}

A conceptual design for the spin rotators and snakes using conventional magnets follows. It is believed that superconducting magnets may be cheaper, since the power consumption for conventional magnets is significant. A conceptual design with superconducting magnets should be considered, possibly using the standard RHIC magnet design.

There are eight spin rotators and two full Siberian Snakes needed for operating RHIC with polarized beams and providing longitudinal polarization at the STAR and the PHENIX interaction region, as shown in Fig. A12. Each of the spin rotators consists of six dipole magnets with the following configuration:

\begin{tabular}{|l|c|c|c|c|c|c|}
\hline Deflection direction & $\mathrm{V}$ & $\mathrm{H}$ & $-2 \mathrm{~V}$ & $-2 \mathrm{H}$ & $\mathrm{V}$ & $\mathrm{H}$ \\
\hline Max. required spin rotation & $45^{\circ}$ & $135^{\circ}$ & $90^{\circ}$ & $270^{\circ}$ & $45^{\circ}$ & $135^{\circ}$ \\
\hline
\end{tabular}

The $-2 \mathrm{H}$ magnet is split into two $135^{\circ}$ sections for simplicity, so that we have only three magnet types $\left(45^{\circ}, 90^{\circ}\right.$, and $\left.135^{\circ}\right)$.

With $\mathrm{B}=2.0 \mathrm{~T}$, we get $0.69 \mathrm{~m}, 1.35 \mathrm{~m}$, and $2.03 \mathrm{~m}$ for the effective length of the magnets. The magnet aperture is $15 \mathrm{~cm} \times 15 \mathrm{~cm}$, which allows for a maximum excursion of $7 \mathrm{~cm}$ and a full $95 \%$ beam width of $8 \mathrm{~cm}$. The magnet height is $58 \mathrm{~cm}$ and the half width is $40.8 \mathrm{~cm}$, so that two magnets fit side by side into the $90-\mathrm{cm}$ wide separation between the two RHIC rings.

The copper coils have 247 turns each and require a current of $1000 \mathrm{~A}$. Following is a list of the magnet parameters:

\begin{tabular}{|c|c|c|c|}
\hline Magnet length & Voltage & Current & Number of Magnets \\
\hline $0.68 \mathrm{~m}$ & $145 \mathrm{~V}$ & $1000 \mathrm{~A}$ & 2 \\
\hline $1.35 \mathrm{~m}$ & $203 \mathrm{~V}$ & $1000 \mathrm{~A}$ & 1 \\
\hline $2.03 \mathrm{~m}$ & $263 \mathrm{~V}$ & $1000 \mathrm{~A}$ & 4 \\
\hline
\end{tabular}

The four horizontal magnets and the three vertical magnets are connected in series, respectively. This requires one $1052 \mathrm{~V} / 1000 \mathrm{~A}$ and one $498 \mathrm{~V} / 1000 \mathrm{~A}$ power supply 
for each spin rotator. Each rotator uses 0.6 MW electric power for radial polarization at the collision point and $1.5 \mathrm{MW}$ for longitudinal polarization.

The two full Type II Siberian Snakes in the 12 o'clock region can be constructed from eight dipole magnets with the following configuration:

\begin{tabular}{|l|c|c|c|c|c|c|c|c|}
\hline Deflection direction & $\mathrm{V}$ & $\mathrm{H}$ & $-\mathrm{V}$ & $-\mathrm{H}$ & $-\mathrm{H}$ & $-\mathrm{V}$ & $\mathrm{H}$ & $\mathrm{V}$ \\
\hline Required spin rotation & $90^{\circ}$ & $90^{\circ}$ & $90^{\circ}$ & $90^{\circ}$ & $90^{\circ}$ & $90^{\circ}$ & $90^{\circ}$ & $90^{\circ}$ \\
\hline
\end{tabular}

The power consumption for each of the full snakes is $1.6 \mathrm{MW}$. The total power consumption ranges from a minimum of 5.6 MW for radial polarization in STAR and vertical polarization in PHENIX, to a maximum of $15.2 \mathrm{MW}$ for longitudinal polarization in both detectors.

Following is a cost estimate with conventional magnets of the eight spin rotators and the two full Siberian Snakes, not including installation and controls:

\begin{tabular}{|l|c|}
\hline Magnets & $\$ 2.1 \mathrm{M}$ \\
Power Supplies & $\$ 6.0 \mathrm{M}$ \\
Cabling & $\$ 0.2 \mathrm{M}$ \\
& \\
\hline Total & $\$ 8.3 \mathrm{M}$ \\
\hline
\end{tabular}

\section{A.4 Schedule and Request}

The partial-snake experiment in the AGS, E-880, will begin running in the spring/summer of 1993. At that time, we will have the required spin hardware, up to injection into RHIC.

To carry out this spin program, we will need to design and construct the RHIC snake magnets described above, and the necessary RHIC polarimeters. We request approval now so that we can proceed with this design and construction work. We believe that with approval now and timely funding, we will be ready to begin this physics program soon after RHIC turns on. 
We have based our physics sensitivity calculations on $4 \times 10^{6}$ seconds, or about 100 days of running at 50\% efficiency. The lower-energy part of our program is compatible with heavy ion running with pp, and we expect that these runs at $\sqrt{\mathrm{s}}=200 \mathrm{GeV}$ would be done with polarized protons. The $\sqrt{\mathrm{s}}=500 \mathrm{GeV}$ running requested is $4 \times 10^{6}$ seconds with $\mathcal{L}=2 \times 10^{32} \mathrm{~cm}^{-2} \mathrm{sec}^{-1}$ luminosity. 


\section{References}

\section{Section A}

1) Proc. of the Polarized Collider Workshop, Penn State University, 1990, edited by J. Collins, S. Heppelmann, and R. W. Robinett (AIP Conf. Proc. No. 223, American Institute of Physics, New York, 1991).

2) C. Bourrely, J. Ph. Guillet, and J. Soffer, Nucl. Phys. B361, 72 (1991);

C. Bourrely, J. Soffer, F. M. Renard, and P. Taxil, Phys. Rep. 177, 319 (1989).

3) G. Bunce, et al., Particle World, Vol. 3, p. 1 (1992).

4) Ya. S. Derbenev and A. M. Kondratenko, Sov. Phys. Doklady 20, 562 (1976);

Ya. S. Derbenev et al., Particle Accelerators, 8, 115 (1978).

5) See A. D. Krisch, Phys. Rev. Lett. 63, 1137 (1989), for the experimetal tests of the Siberian Snake concept.

6) See e.g., S. Y. Lee and E. D. Courant, Phys. Rev. D41, 292 (1990), and references therein; S. Y. Lee and E. D. Courant, Brookhaven preprint AD/RHIC-63.

7) S. Y. Lee, p. 30 in Ref. 1, and references therein.

8) G. Bunce, p. 147 in Ref. 1.

9) E. L. Berger and J. Qiu, Phys. Rev. D40, 778 (1989).

10) E. Richter-Was, Phys. Rev. D34, 2893 (1986).

11) X. Ji, Phys. Lett. B284, 137 (1992).

12) J. Ralston and D. E. Soper, Nucl. Phys. B152, 109 (1979).

13) J. Kodaira et al., Nucl. Phys. B159, 99 (1979); A. P. Bukhvostov et al., Sov. Phys. JETP 60, 22 (1984).

14) X. Artru and M. Mekhfi, Z. Phys. C45, 669 (1990).

15) J. Collins, Penn State Preprint.

16) J. L. Cortes, B. Pire, and J. P. Ralston, p. 184 in Ref. 1.

17) R. L. Jaffe and X. Ji, Phys. Rev. Lett. 67, 552 (1991). 
18) J. Qiu and G. Sterman, Phys. Rev. Lett. 67, 2264 (1991).

19) X. Ji, Phys. Lett., to appear.

20) J. L. Cortes, B. Pire, and J. P. Ralston, Ecole Polytechnique Preprint A048, 0491 (1991).

21) R. L. Jaffe and X. Ji, MIT CTP Preprint No. 2005; Nucl. Phys. B, to appear.

22) M. J. Tannenbaum, p. 201 in Ref. 1, and references therein.

23) They were obtained from the parametrizations of the distributions used in Ref. 2.

24) V. Barger, W. Y. Keung, and R. J. N. Phillips, Z. Phys. C6, 169 (1980);

D. W. Duke and J. F. Owens, Phys. Rev. D30, 49 (1984).

25) M. Gluch, J. F. Owens, and E. Reya, Phys. Rev. D17, 2324 (1978); H. Fritzsch, Phys. Lett. B67, 217 (1977).

26) V. Barger and A. D. Martin, Phys. Rev. D31, 1051 (1985).

27) (UA1 Collaboration) C. Albajar et al., Phys. Lett. B186, 237 (1987); Phys. Lett. B256, 112 (1991).

28) J. L. Cortes and B. Pire, Phys. Rev. D38, 3586 (1988).

29) M. A. Doncheski and R. W. Robinett, Phys. Lett. B248, 188 (1990).

30) R. W. Robinett, Phys. Rev. D43, 113 (1991).

31) J. Soffer and N. Törnqvist, Phys. Rev. Lett. 68,907 (1992).

32) J. G. Alessi et al., AIP Conf. Proc. No. 187, p. 1221 (1988).

33) F. Z. Khiari et al., Phys. Rev. D39, 45 (1989).

34) T. Roser, AIP Conf. Proc. No. 187, p. 1442 (1988).

35) S. Y. Lee and E. D. Courant, BNL Technical Note AD/RHIC-63.

36) S. Y. Lee and S. Tepikian, Phys. Rev. Lett. $\underline{56}, 1635$ (1986).

37) S. Y. Lee, Nucl. Inst. Methods $\underline{\mathrm{A} 306}, 1$ (1991).

38) T. Roser, Spin Rotators and Split Siberian Snakes, submitted to Particle Accelerators.

39) Conceptual Design of the RHIC, BNL 52195 (1989). 
40) V. A. Anferov et al., IUCF Newsletter No. 50, p. 11 (1992).

41) J. Schwinger, Phys. Rev. 73, 407 (1948).

42) See for instance, C. Bourrely and J. Soffer, Nuovo Cimento Lett. 19, 569 (1977).

43) N. Akchurin et al., Phys. Lett. B229, 299 (1989), and references therein.

44) B. Z. Kopeliovich and L. I. Lapidus, Yad. Fiz. 19, 218 (1974); [(Sov. J. Nucl. Phys. 19, 114 (1974)].

45) N. H. Buttimore, E. Gotsman, and E. Leader, Phys. Rev. D18, 694 (1978).

46) N. H. Buttimore, Proc. 6th Int. Symp. on High Energy Spin Physics, Marseille, Ed. J. Soffer, Journal de Physique 46, C2 643 (1985).

47) N. Akchurin et al., to be published.

48) D. L. Adams et al., Phys. Lett. B264, 462 (1991). 


\section{Figure Captions}

\section{Section A}

Figure 1 Diagram of parton subprocesses in QCD. Hadrons a and b contain partons $i$ and $j$, which interact with cross section $\hat{\sigma}_{i j}$.

Figure 2 The asymmetries $\hat{a}_{\mathrm{LL}}^{\mathrm{j}}$ versus the c.m. scattering angle $\theta^{*}$ at the lowest-order perturbative QCD for different subprocesses.

Figure 3 Compilation of all data on $A_{1}^{P}$ as a function of $x$.

Figure 4 Predicted two-spin asymmetries for jet production as a function of $\mathrm{pT}_{\mathrm{T}}$ with standard $\Delta \mathrm{G}$ (dotted) and a large $\Delta \mathrm{G}$ (solid), which is inspired by the EMC result.

Figure 5 Predicted ALL at $\sqrt{\mathrm{s}}=300 \mathrm{GeV}$ as a function of the jet pair mass for a standard $\Delta G$ (dashed curve) and a large positive $\Delta G$ (solid curve).

Figure 6 Predicted direct photon two-spin asymmetry as a function of $\mathrm{p}_{\mathrm{T}}$ for large $\Delta \mathrm{G}$ at $45^{\circ}$ (solid), $90^{\circ}$ (dashed), and for standard $\Delta \mathrm{G}$ (dotted).

Figure 7 The ratio of the observable asymmetry ATT with the parton asymmetry $\hat{a}_{\mathrm{TT}}$ as a function of $\mathrm{x}_{\mathrm{F}}$ for the Drell-Yan process in polarized pp collisions at $\sqrt{\mathrm{s}}=100 \mathrm{GeV}$. The mass of the lepton pairs is fixed at $7 \mathrm{GeV}$ for the solid curve and $15 \mathrm{GeV}$ for the dashed curve.

Figure 8 Predictions of $\mathrm{g}_{1}(\mathrm{x})$ and $\mathrm{h}_{1}(\mathrm{x})$ from the bag model.

Figure 9 Single helicity asymmetries versus rapidity y for $\mathrm{W}^{+}$and $\mathrm{W}^{-}$.

Figure 10 Single helicity asymmetry versus rapidity y for $Z^{\circ}$. 
Figure 11 Diagram of the proposed spin RHIC complex.

Figure 12 Top: $\quad$ RHIC insertion region showing the empty straight sections on either side of the interaction point.

Bottom: Modified RHIC insertion region with straight sections parallel to the interaction region. Also shown is the schematic layout of the spin rotators.

Figure 13 Lumonisity versus energy for proton-proton collisions in RHIC. Conditions for the "enhanced" luminosity are described in the text.

Figure 14 Adiabatic spin reversal of a stored beam of polarized protons at the IUCF cooler ring. The RF voltage is proportional to the strength of the artificial spin resonance driving the spin reversal.

Figure 15 Analyzing power $\mathrm{A}_{\mathrm{N}}$ of proton-proton scattering in the Coulombnuclear interference region as a function of the momentum transfer, $t$.

Figure $16 \quad A_{N}$ versus $x_{F}$ for $\pi^{+}, \pi$, and $\pi^{\circ}$ FNAL data at $200 \mathrm{GeV}$. 


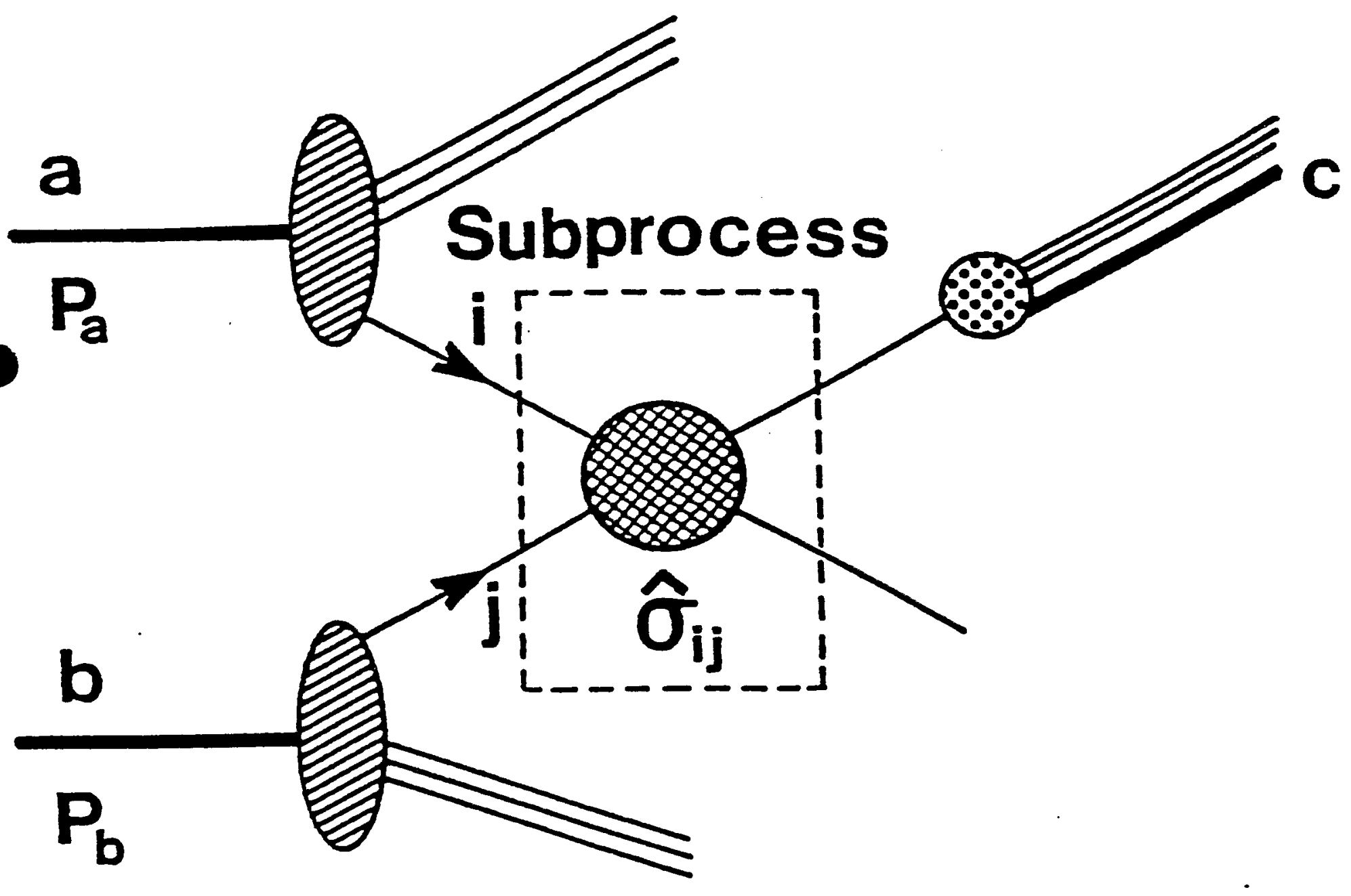

Figure Al 


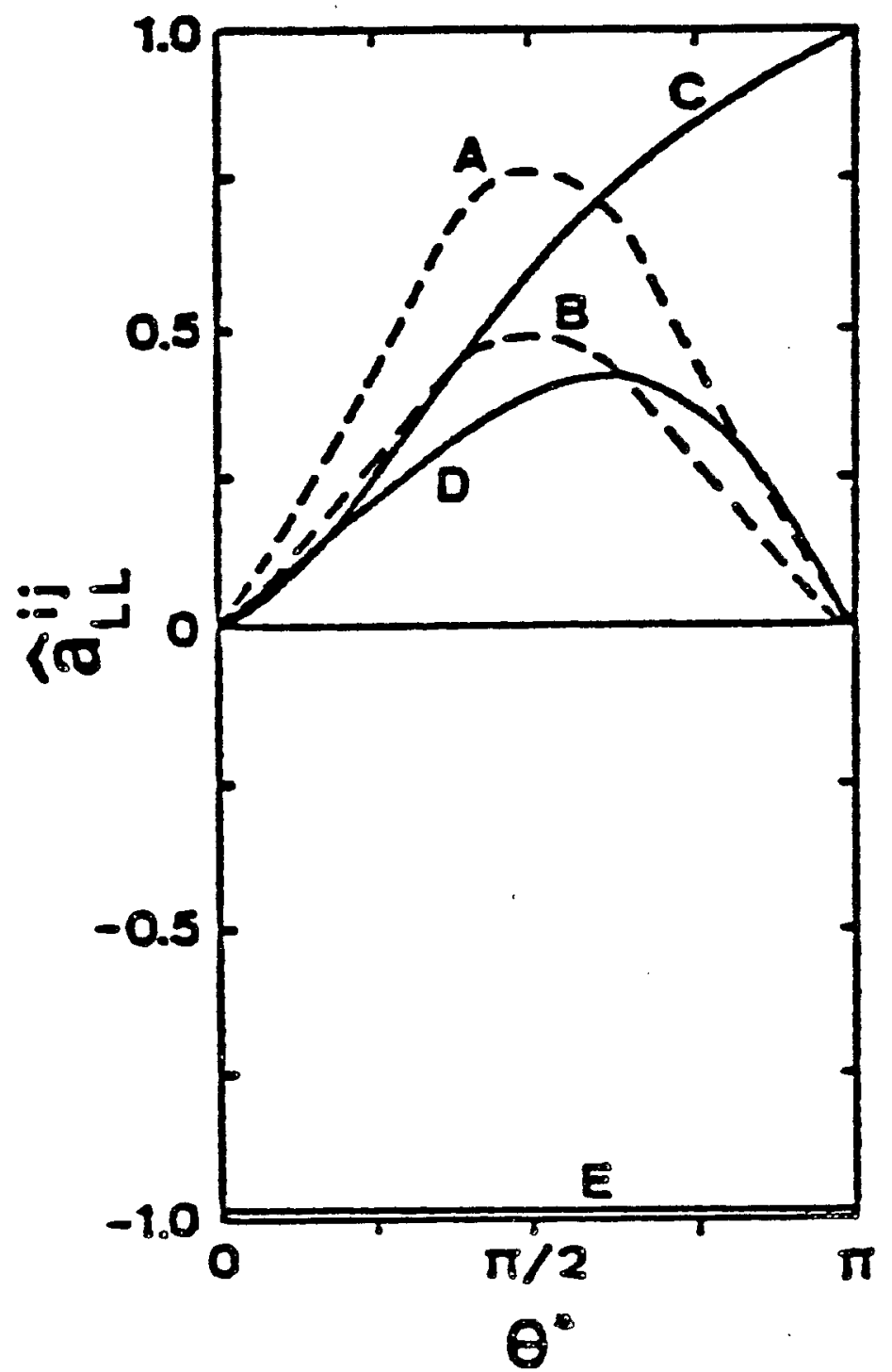

$A[g g-g g$
$B\left[\begin{array}{l}d d-d d \\ u u-u\end{array}\right.$
$c\left[\begin{array}{l}u d-u d \\ u \bar{d}-u \bar{d} \\ d u \bar{u}-d \bar{u} \\ q g-q g \\ q g-9 r\end{array}\right.$

$D\left[\begin{array}{l}u \bar{u}-u \bar{u} \\ d \bar{d}-d \bar{d}\end{array}\right.$

$E\left[\begin{array}{l}9 g-9 \bar{q} \\ q \bar{q}-99 \\ u \bar{u}-d \bar{d} \\ d \bar{d}-4 \bar{u} \\ q \bar{q}-98\end{array}\right.$

Figure A2 


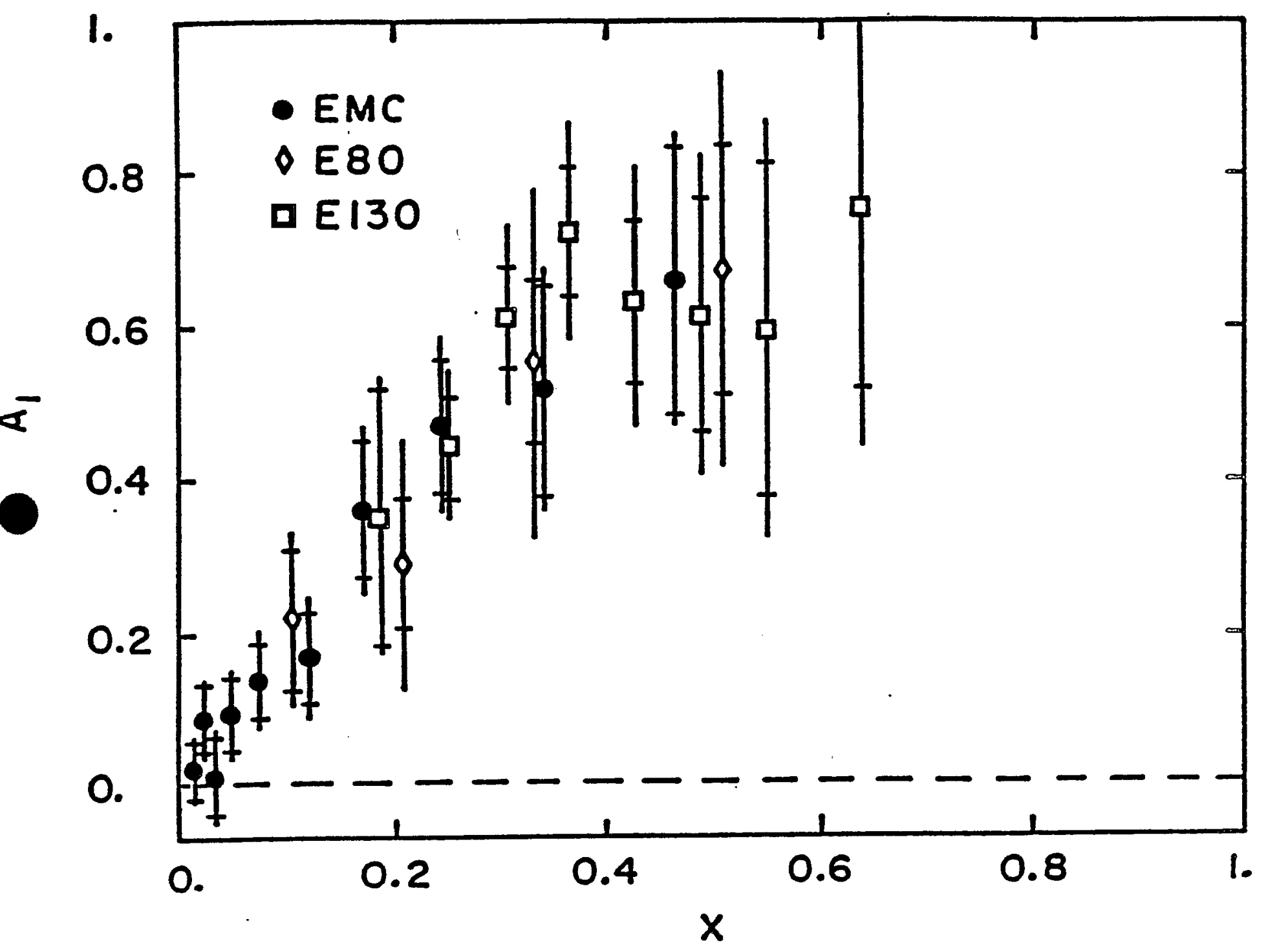

Figure A3 


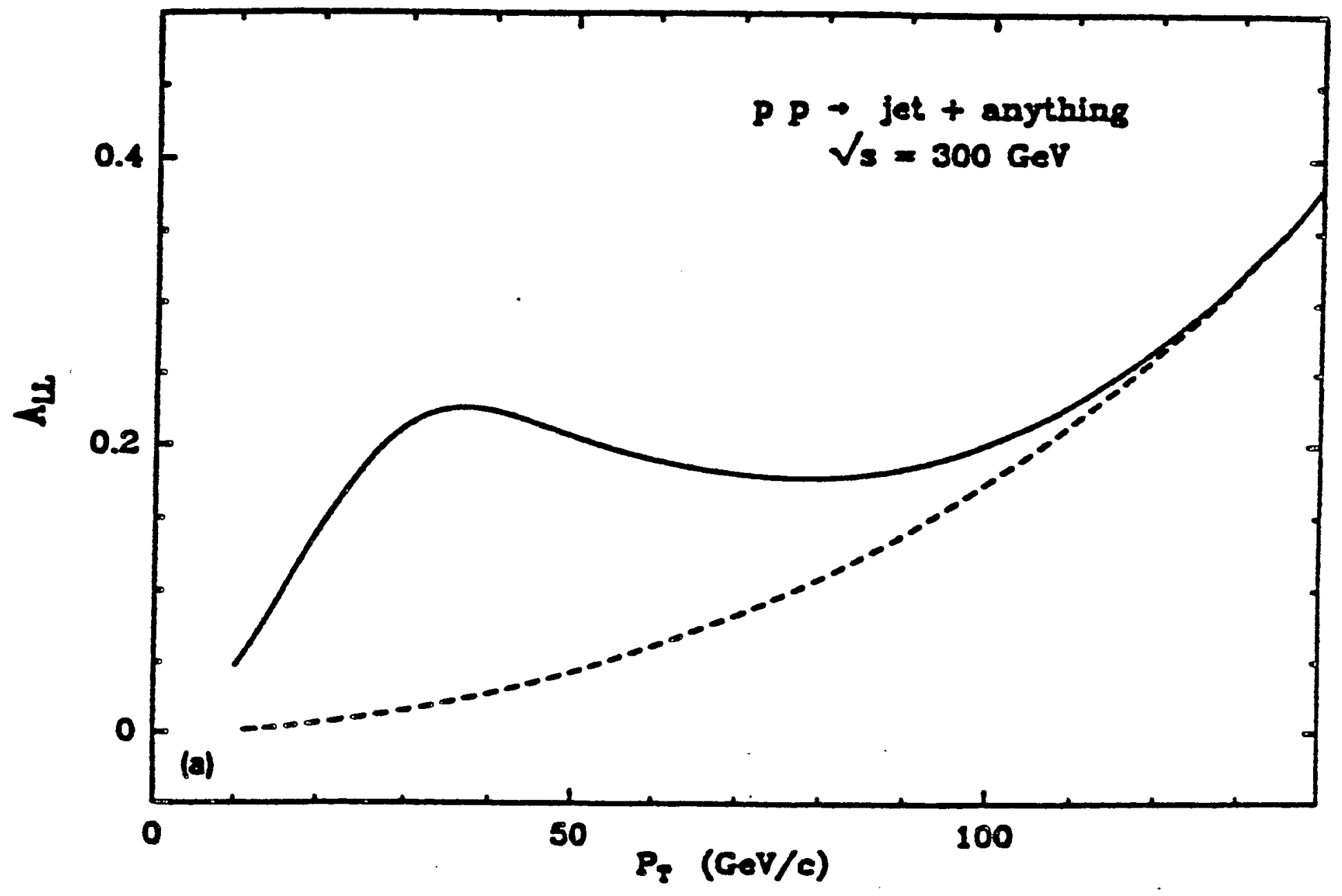

Figure 44 


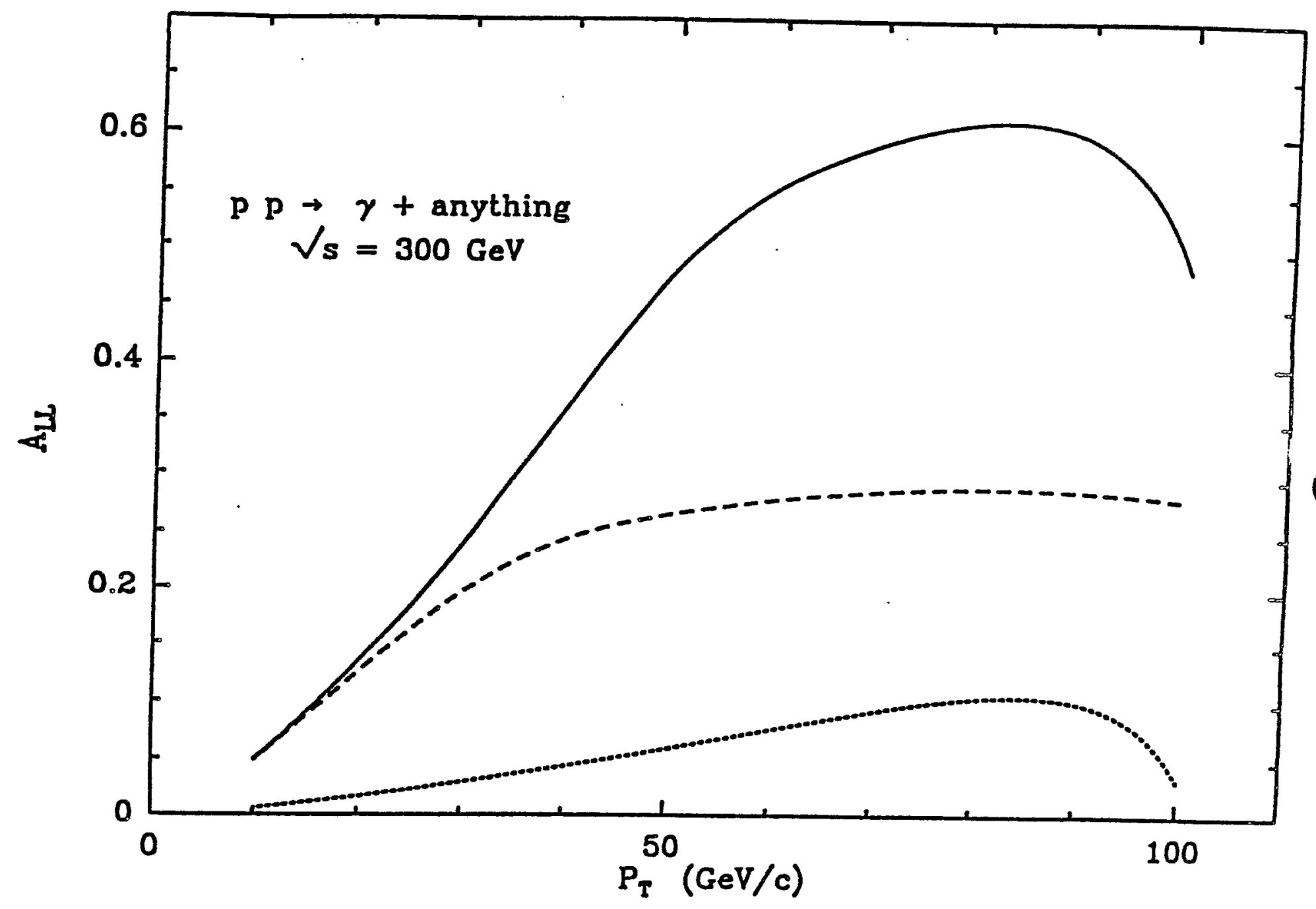

Figure A6 


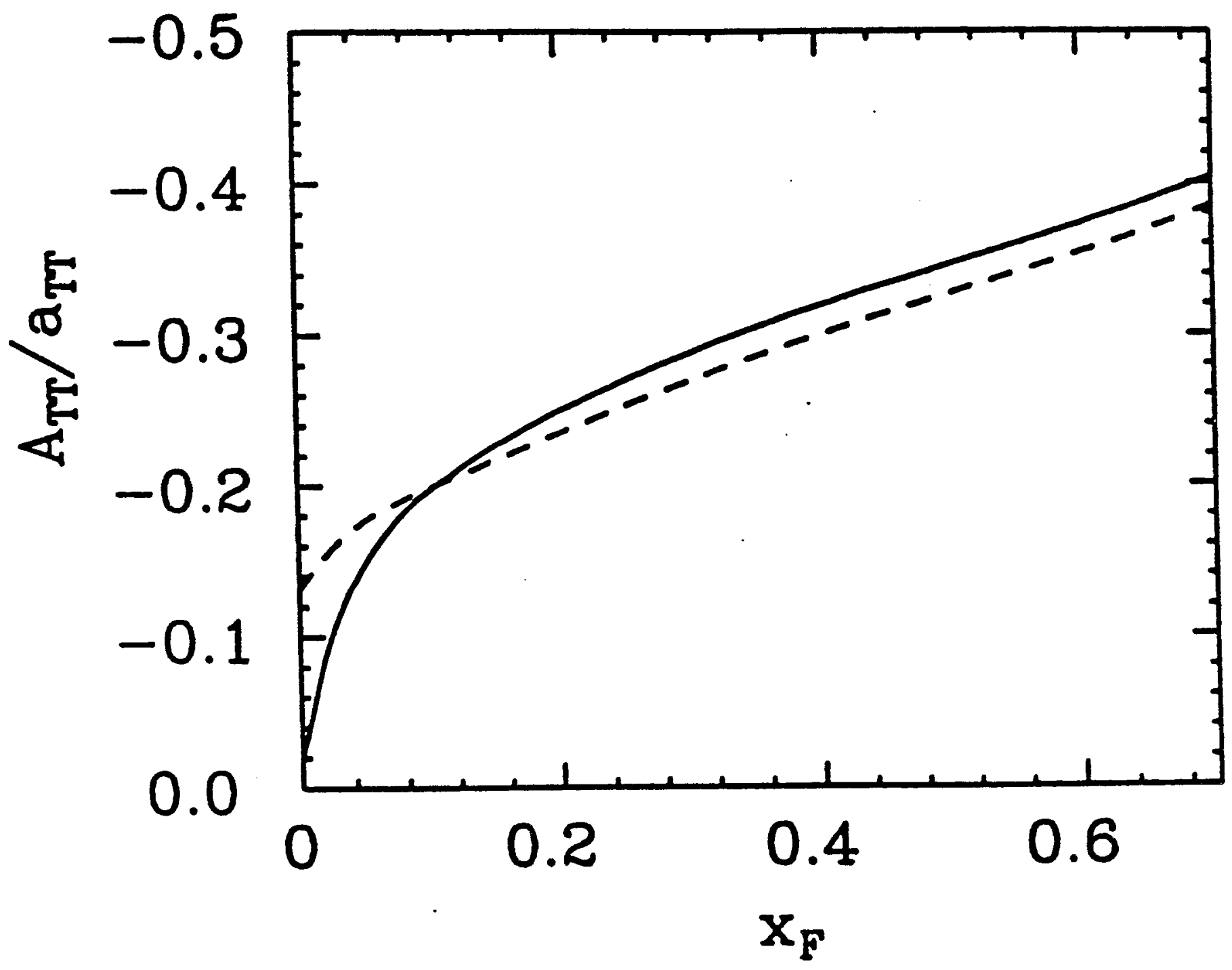

Eigure A7 


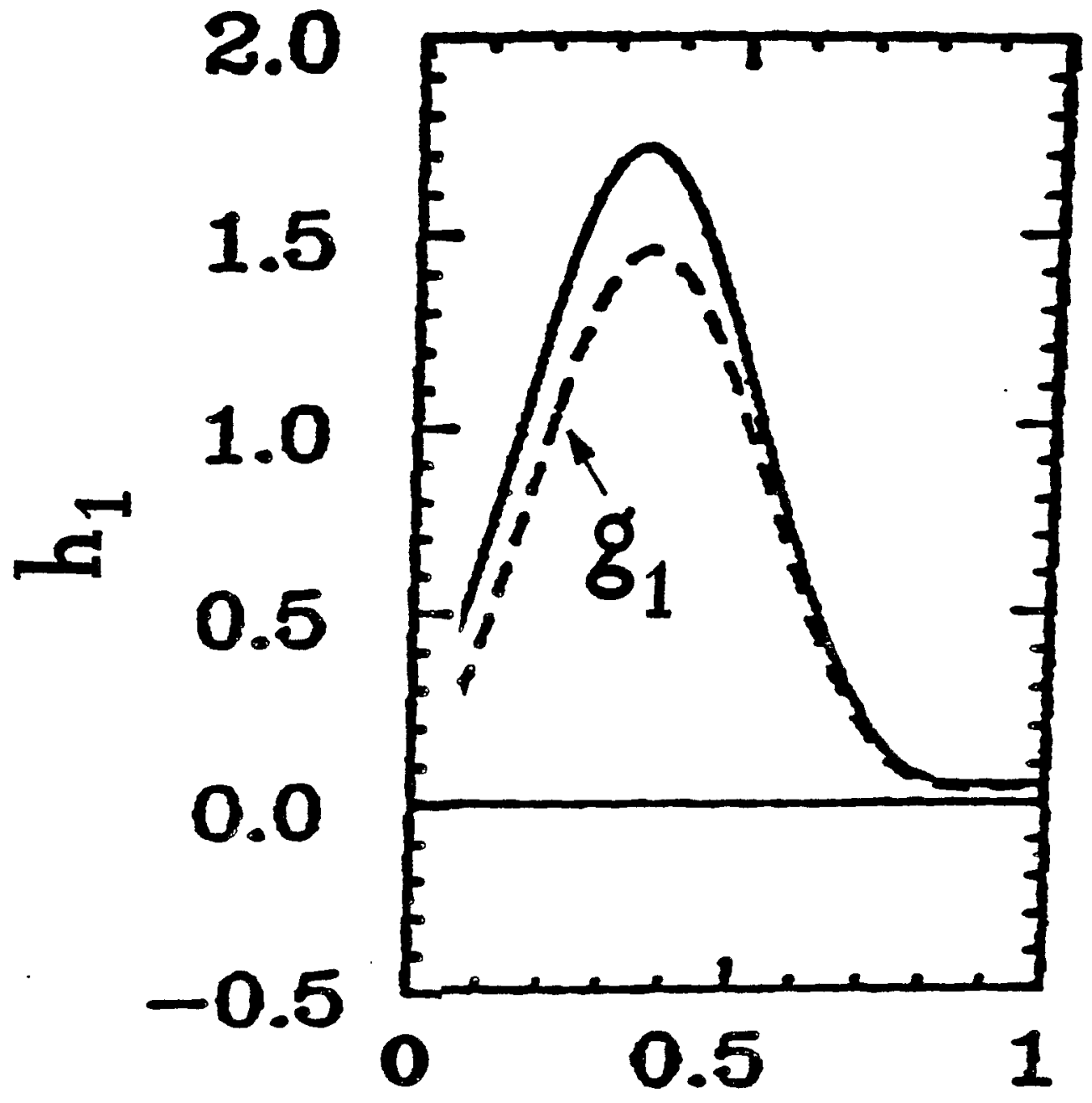

Figure A8 


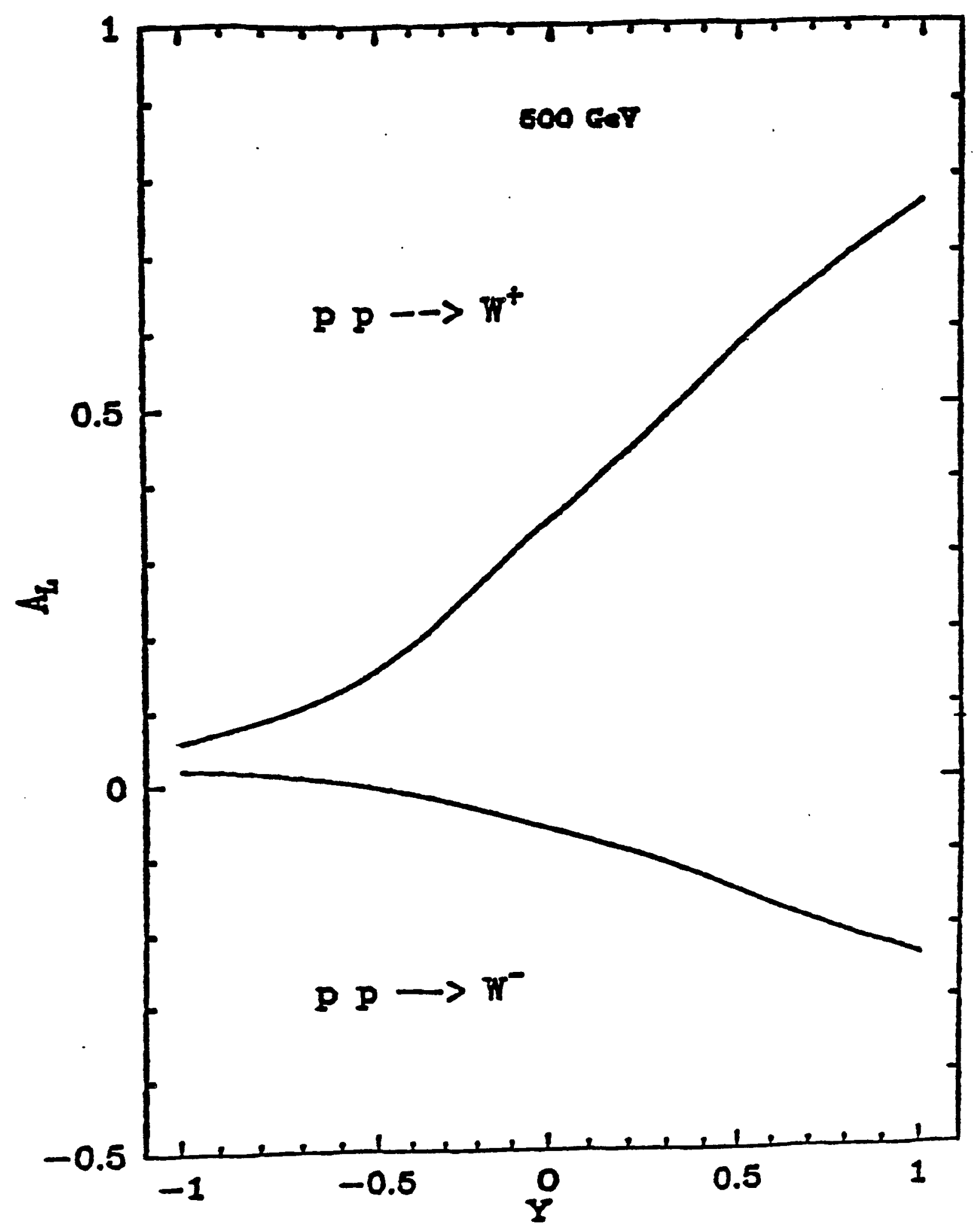

Figure A9 


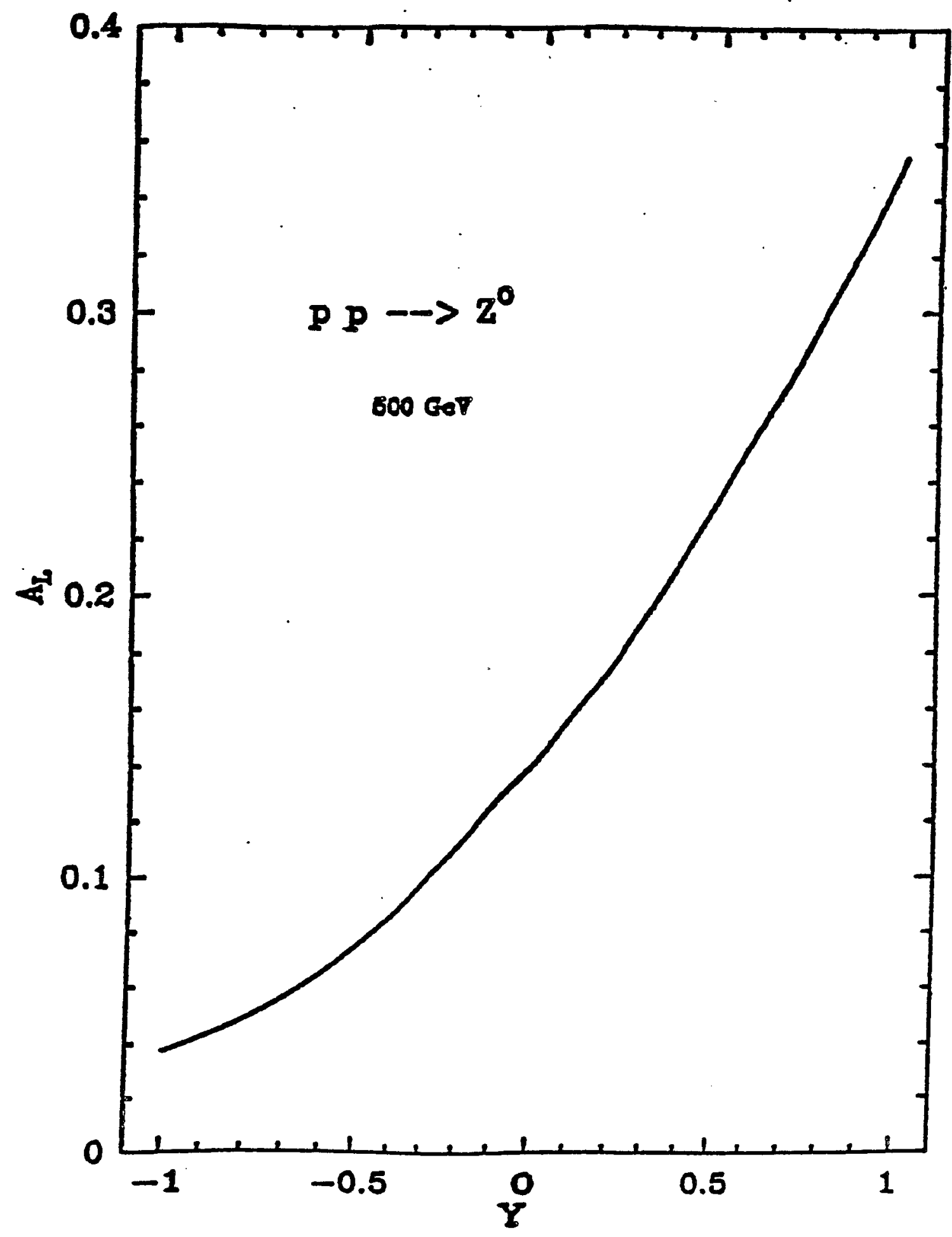

Figure AlO 
POLARIZED PROTON COLLISIONS AT BROOKHAVEN

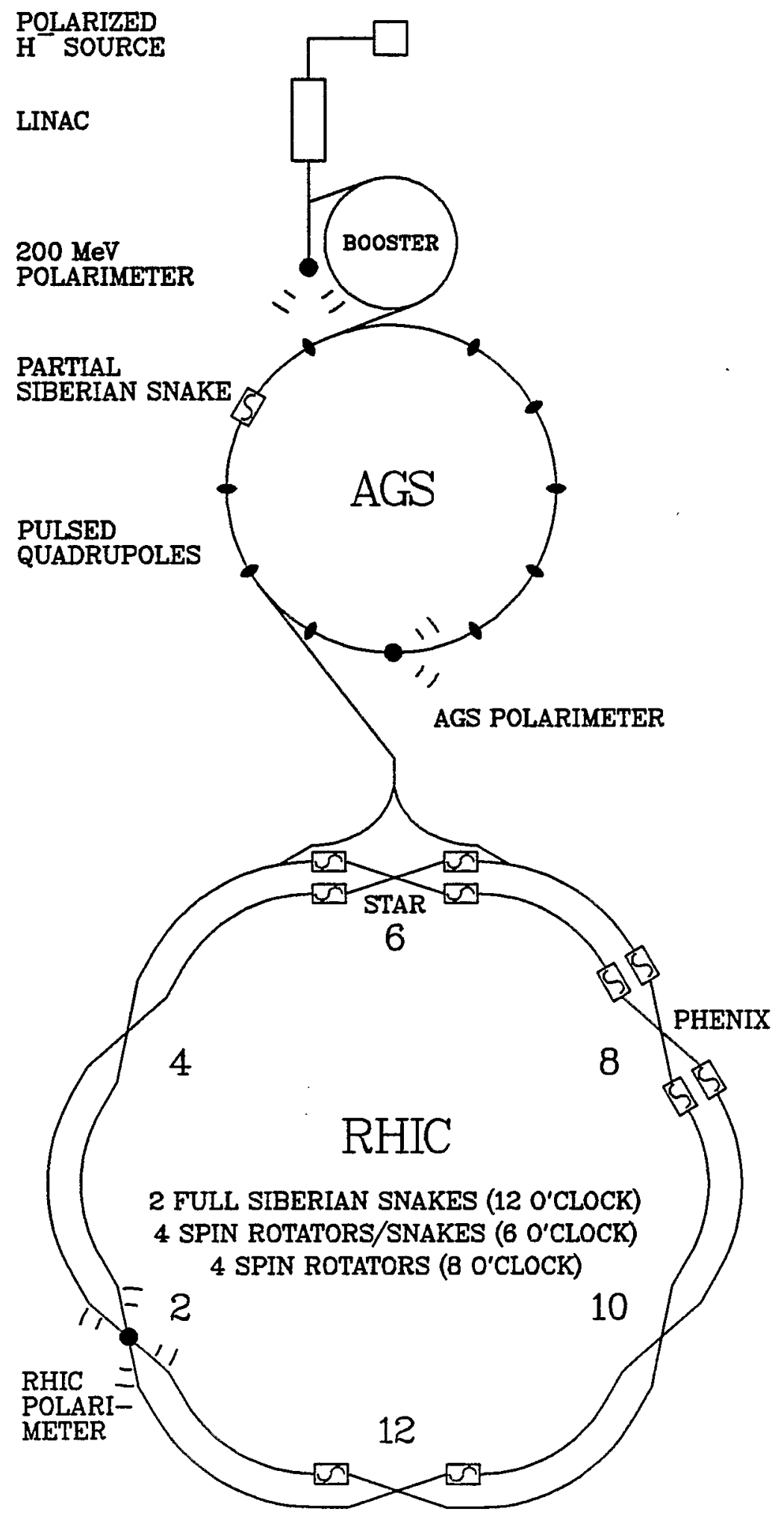

Figure A.11 

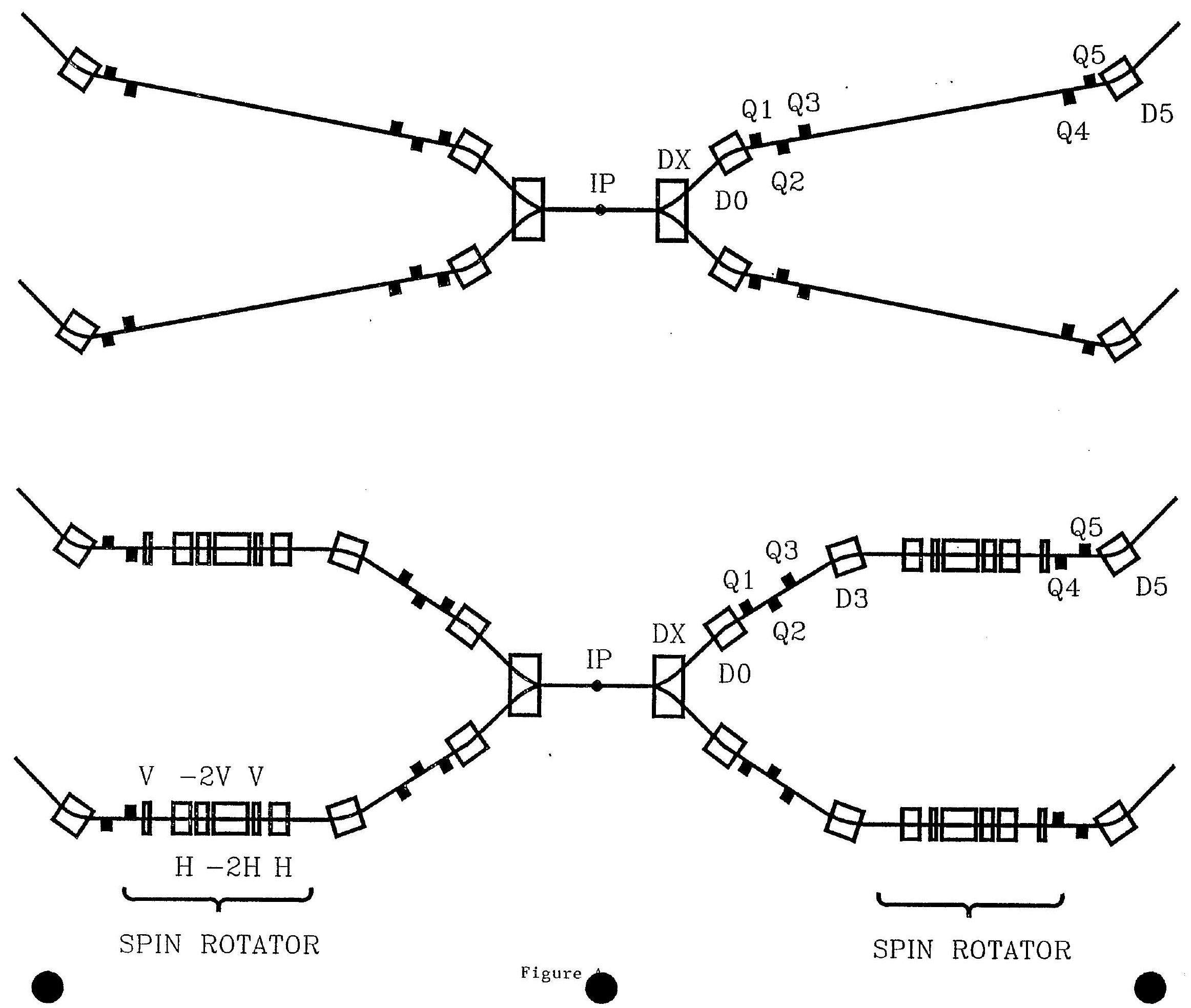


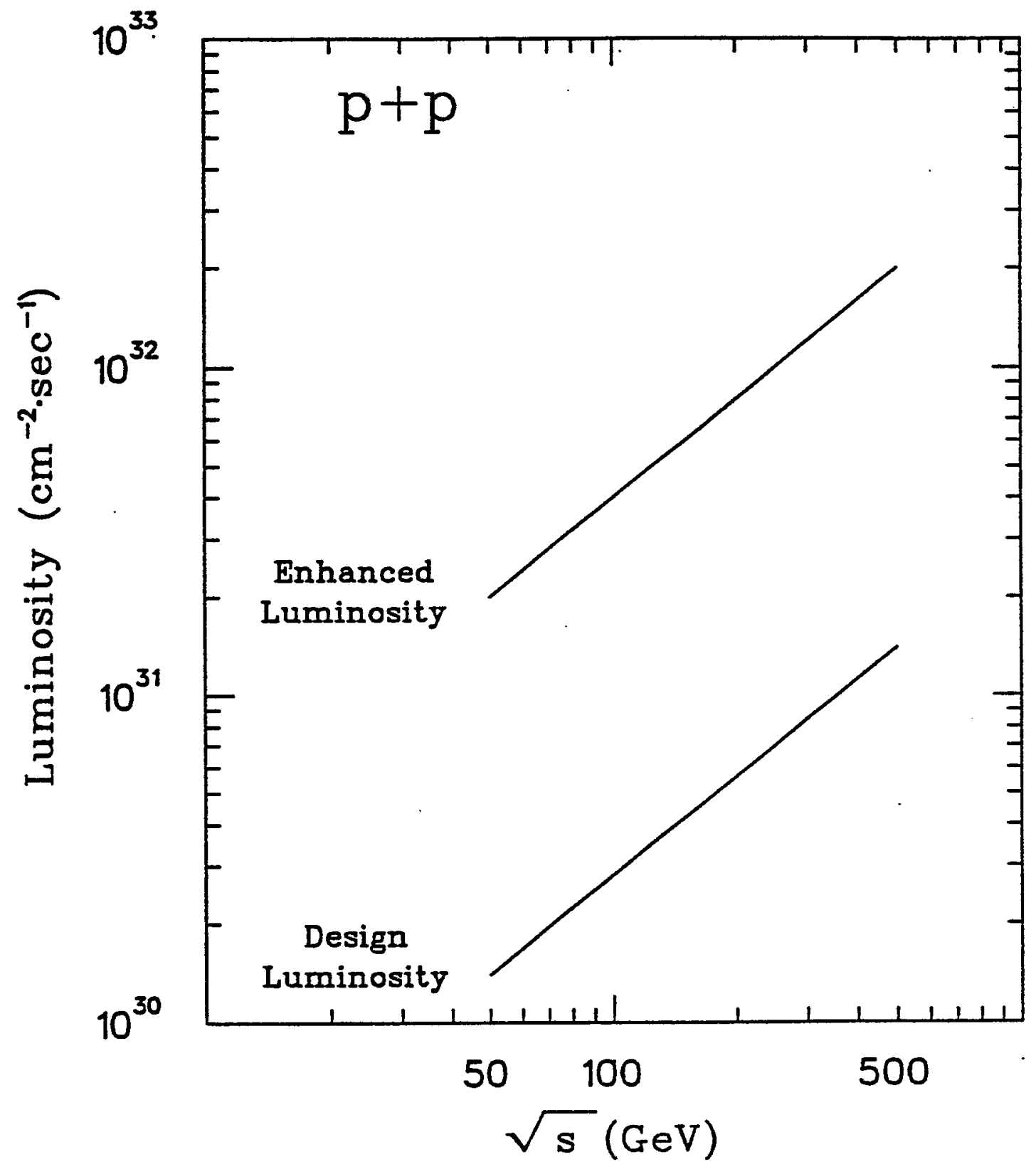

Figure A13 


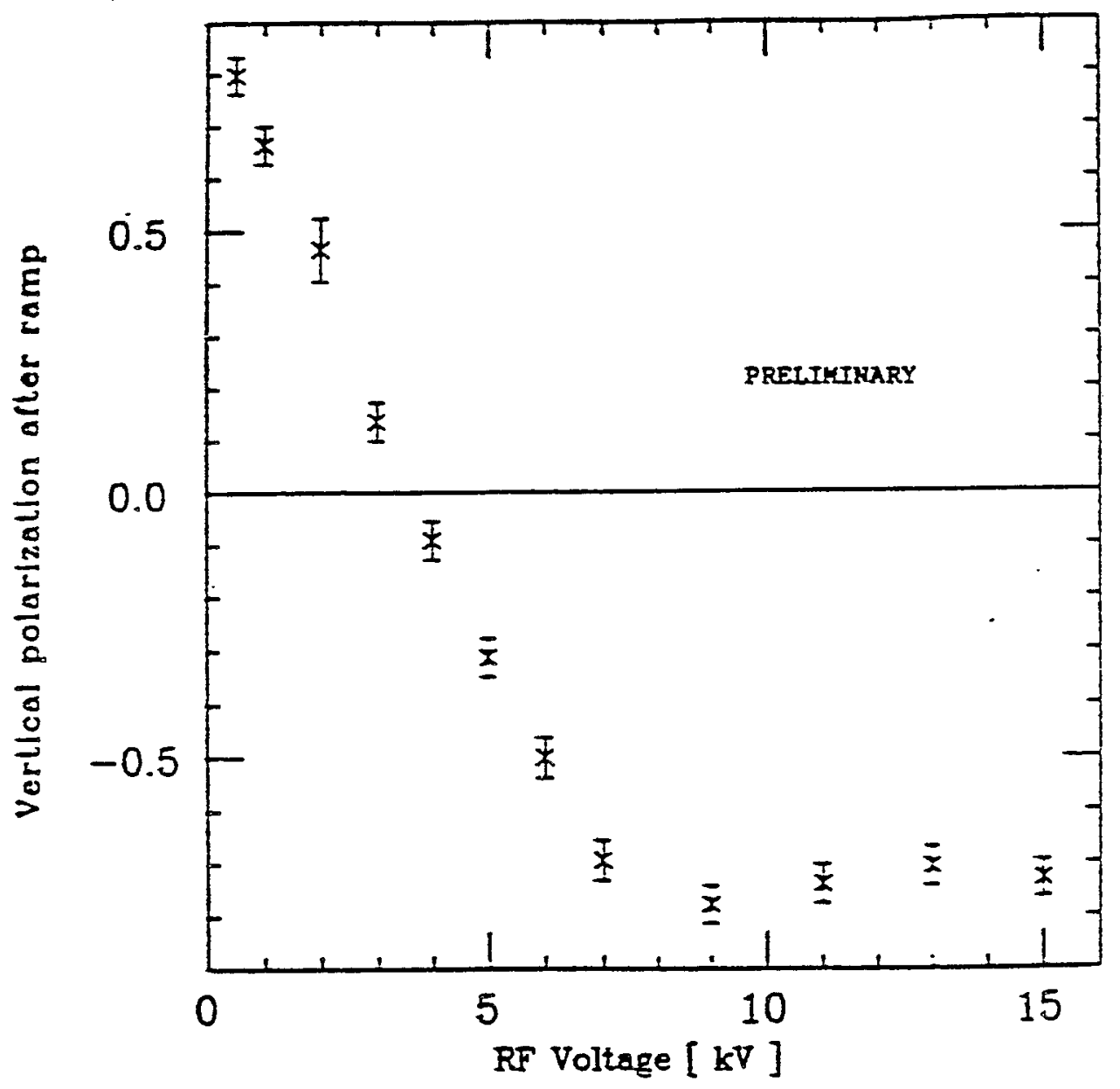

RF freq. ramp $1.509 \rightarrow 1.507 \mathrm{MAl}=$ ramp time 30 msec

Figure A.14 


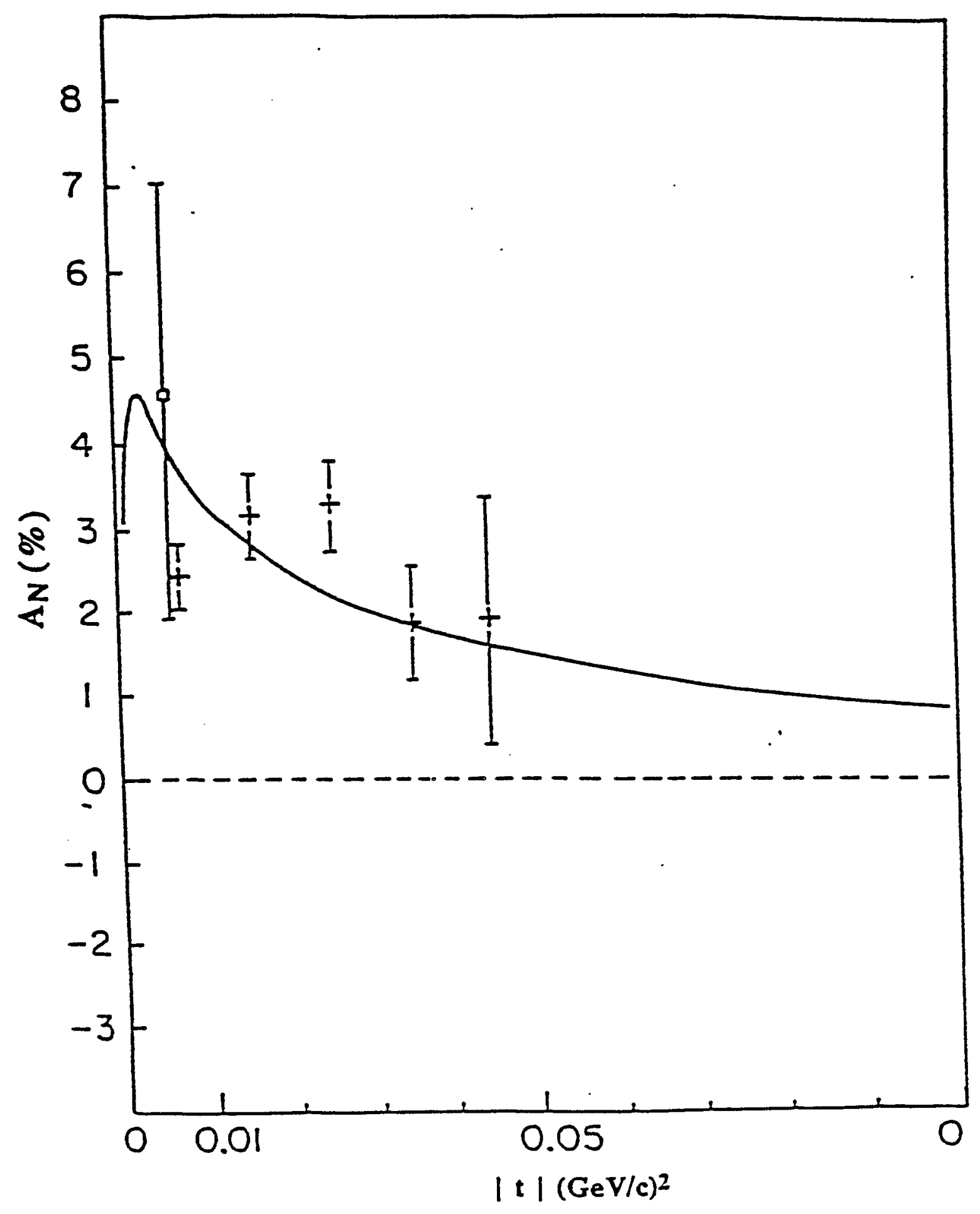

Figure A15 


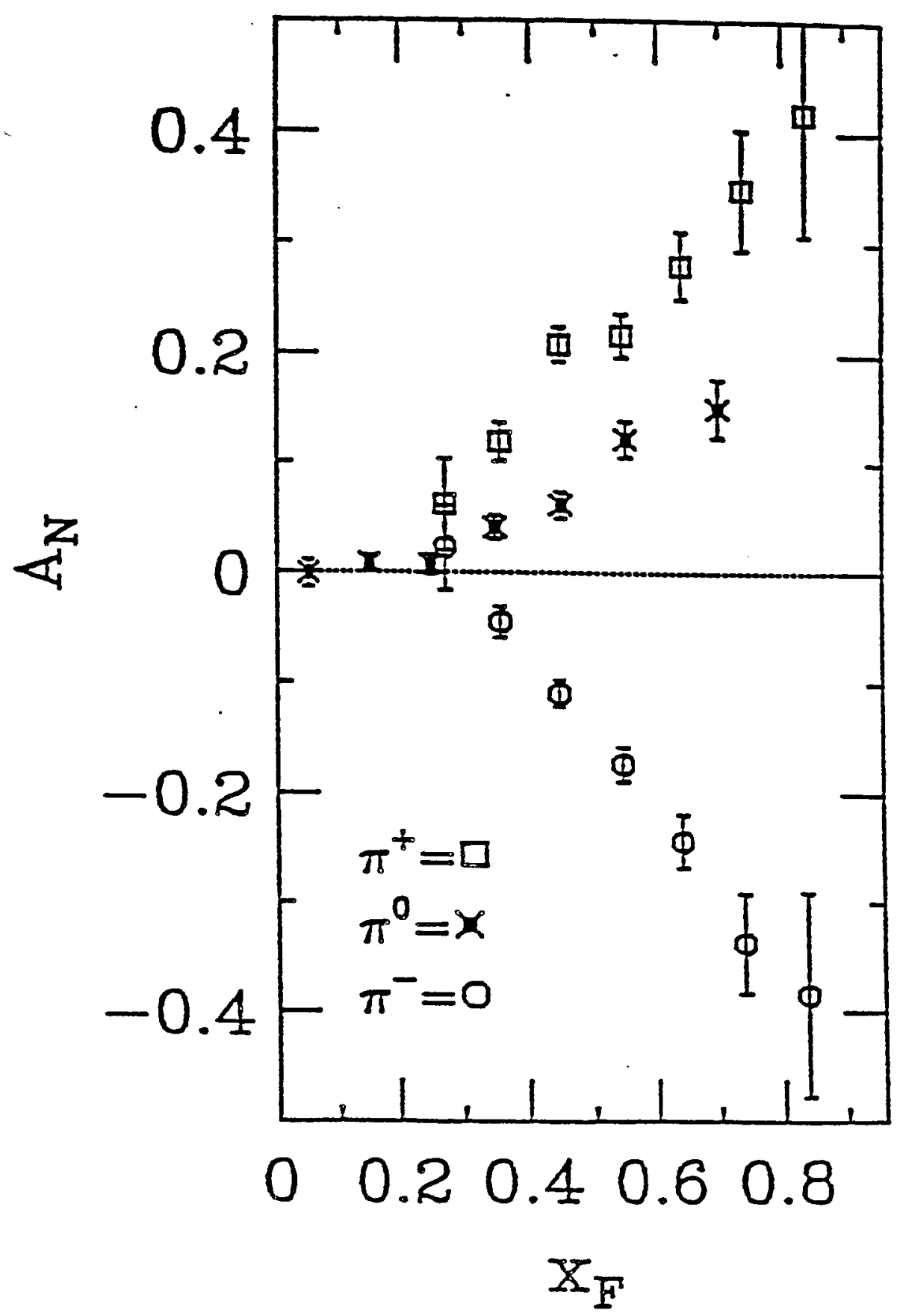

Figure Al6 


\section{Section B Experiments with the PHENIX Detector}

\section{by The PHENIX/SPIN Collaboration}

- Brookhaven National Laboratory, Upton, $N Y 11973$

S. H. Aronson, M. Fatyga, S. Gavin, W. Guryn*, J. Harder, S. Kahn, E. Kistenev, P. Kroon, Y. Makdisi, M. J. Murtagh, E. O'Brien, L. Paffrath, S. Rankowitz, S. Rescia, T. K. Shea, M. Tanaka, M. J. Tannenbaum, C. L. Woody

- University of California - Riverside, Riverside, CA 92521

P. Beery, Sun-Yiu Fung*, Ju-Hwan Kang, R. Seto

- China Institute of Atomic Energy, Beijing, $P$. R. China Xixiang Bai, Kexing Jing, Xiaoping Liu, Zuhua Liu, Yajun Mao, Benhao Sa, Zuxun Sun*, Yude Wan, Zhongqi Wang, Jincheng Xu, Xiaoze Zhang, Yuming Zheng, Shuhua Zhou

- Columbia University, Nevis Labs., Irvington, $N Y 10533$

C. Y. Chi, B. Cole, J. Dodd, Qiming Li, S. Nagamiya, T. Nayak, W. Sippach, P. Stankus, O. Vossnack, Fuqiang Wang, Yufeng Wang, Yuedong Wu, Xihong Yang, W. A. Zajc*, Wenlong Zhan

re

- Hiroshima University, Saijo, Hiroshima, Japan

H. Iwata, A. Sakaguchi, T. Sugitate, Y. Sumi*

- Institute of High Energy Physics, Protovino, Russia

V. V. Ammosov, A. Denisov, A. Durum, V. Gapienko, Yu. Galitsky, Yu. Gutnikov, A. Ivanilov, V. I. Kochetkov, B. Korablev, A. Kozelov, V. V. Makeev, E. Melnikov, Yu. Mikhailov, V. Onuchin, Yu. Pischalnikov, Yu. Protopopov, V. I. Rykalin, K. Shestermanov, A. Starkov, D. Strustyumov, A. Surkov, V. Zaetz, A. Zaichenkov

- Institute for Nuclear Study, (U. Tokyo), Tanashi, Tokyo, Japan

Y. Akiba, H. Hamagaki*, S. Homma, H. Sako, M. Sekimoto

- KEK, Institute for High Energy Physics, Tsukuba, Japan

J. Chiba*, Y. Mori, M. Nomachi, O. Sasaki, H. Sakamoto, T. Shintomi, K. H. Tanaka

- Kyoto University, Kyoto, Japan

H. Enyo*, K. Imai, H. Kaneko, A. Masaike

- Los Alamos National Laboratory, Los Alamos, NM 87545

J. G. Boissevain, T. A. Carey, A. Gavron, H. van Hecke, B. Jacak*, J. Kapustinsky, M. Leitch,

J. Lillberg, P. L. McGaughey, J. Moss, J. Simon-Gillo, W. E. Sondheim, J. P. Sullivan,

L. Waters

- Lund University, Lund, Sweden

S. Garpman, H.-A. Gustafsson, A. Oskarsson*, I. Otterlund, K. Soderstrom,

E. Stenlund 
- McGill University, Montreal, Quebec, Canada

J. Barrette, S. K. Mark*, L. Nikkinen, L. Normand, M. Rosati

- National Institute of Radiation Science, Chiba-ken, Japan

E. Takada*

- St. Petersburg Nuclear Physics Institute, Gatchina, Leningrad, Russia

N. K. Abrosimov, N. N. Chernov, V. G. Ivochkin, L. M. Kochenda, G. A. Rjabov, V. Schegelski, D. M. Seliverstov, N. Smirnoff, E. M. Spiridenkov, A. Vorobyov*

- University of Tokyo, Tokyo, Japan

R. Hayano*, T. Ishikawa, H. Sakurai, K. Shigaki, H. Tamura

- University of Tsukuba, Tsukuba, Japan

I. Arai, Y. Igarashi, T. Ikeda, M. Ise, S. Kato, H. Kitayama, A. Kumagai,

K. Kurita, Y. Miake*, Y. Nagasaka, H. Tobinai, K. Tomizawa, S. Ueno, K. Waki, K. Yagi,

Y. Yamashita

- Vanderbilt University, Nashville, TN 37235

E. Cornell, C. Maguire*, A. K. Ramayya

- Individual Participation,

W. L. Kehoe (M.I.T.)

The membership above is tentative. The sign-up procedure is going on for the time being.

* Contact person for each institution.

Representative: H. En'yo

Local coordinators: $Y$. Makdisi

M.J. Tannenbaum 


\section{B.1 Physics Goals and Capabilities}

PHENIX, in its standard configuration[1], is a very high granularity, high resolution, electron, photon and charged hadron spectrometer, in the central region $|\eta| \leq 0.35$, with full azimuth di-muon measurement in one endcap, $1.1 \leq \eta \leq 2.5$. The electron/photon central spectrometer emphasizes electron identification at the trigger level, with RICH, TRD and EM calorimetry. The EM calorimeter also serves as an excellent gamma and $\pi^{0}$ trigger because of its 5 by $5 \mathrm{~cm}^{2}$ segmentation at $5.1 \mathrm{~m}$. The central spectrometer consists of two arms, each subtending $90^{\circ}$ in azimuth $(\Phi)$ and \pm 0.35 units in pseudorapidity $(\eta)$. The total coverage is $1 / 2$ of the azimuth-however, the two arms are not back-to-back: the gap between the edges of the two $90^{\circ}$ arms is $45^{\circ}$ on one side and hence $135^{\circ}$ on the other. The EM calorimeter, $\sigma_{E} / E=7 \% / \sqrt{E(\mathrm{GeV})}$, has a small, high resolution, $\sigma_{E} / E=2 \% / \sqrt{E(\mathrm{GeV})}$, subsection in one $90^{\circ}$ arm, which covers $|\eta| \leq 0.05$. The charged particle momentum resolution is $1 \%$ at $5 \mathrm{GeV} / \mathrm{c}$, and charged hadron identification is provided by $\operatorname{TOF}(\sigma=100 \mathrm{ps})$ for $1 / 3$ of the azimuth of one arm.

The strengths of the PHENIX detector, notably the ability to run at the highest luminosities with very selective triggers, allow measurements of a wide variety of spin phenomena:

- $W^{+} \rightarrow e^{+} \nu$, with the electron detected in the central detector. The parity violating spin asymmetry in the production channel will be measured for the first time with real $W$ 's, via parity violating longitudinal spin asymmetry;

$$
A_{L L}^{P V}=\frac{1}{P_{\text {beam }}^{2}} \frac{N(++)-N(--)}{N(++)+N(--)}
$$

- Direct Photon production with $10 \leq p_{T} \leq 25 \mathrm{GeV} / \mathrm{c}$. This is a measurement of the spin dependent gluon structure function with longitudinally polarized protons, via longitudinal spin asymmetry;

$$
A_{L L}=\frac{1}{P_{\text {beam }}^{2}} \frac{N(++)+N(--)-N(+-)-N(-+)}{N(++)+N(--)+N(+-)+N(-+)}
$$

- Jets via leading $\pi^{0}$, with identified $\pi^{0}$ up to $25 \mathrm{GeV} / \mathrm{c}$ and unresolved clusters above $25 \mathrm{GeV} / \mathrm{c}$. Here both longitudinal and transverse spin asymmetries will be examined. The large cross section of $\pi^{0}$ production enables us to check the parity violation in hadron interactions due to strong-weak interference.

- Drell-Yan in the muon-pair channel and the barrel, with $2 \leq m \leq 3 G e V / c^{2}$ and for higher masses. Transverse and longitudinal spin asymmetries will be measured and the correlation between the spin axis and the angular distribution of muon-pair will be studied.

- $J / \Psi$ and $\chi_{2}$ production. Here the open geometry of PHENIX is exploited: gamma rays of a few hundred $\mathrm{MeV}$ are detected in the high resolution gamma detector in coincidence with $J / \Psi \rightarrow l^{+} l^{-}$. The spin dependent gluon structure function can be measured, and the production mechanism of charmed particles will be studied with additional spin information. 
For the detailed discussion of rates and sensitivities which follows, the "enhanced" luminosity will be taken as $\mathcal{L}=2 \times 10^{32} \mathrm{~cm}^{-2} \mathrm{sec}^{-1}$ at $\sqrt{\mathrm{s}}=500 \mathrm{GeV}$ and $\mathcal{L}=8 \times 10^{31} \mathrm{~cm}^{-2} \mathrm{sec}^{-1}$ at $\sqrt{s}=200 \mathrm{GeV}$. We use 100 days of polarized proton runs with a duty factor of $\sim 50 \%\left(4 \times 10^{6}\right.$ $\mathrm{sec}$ ), which leads to the integrated luminosity of $\int \mathcal{L} d t=8 \times 10^{38} \mathrm{~cm}^{-2}$ and $\int \mathcal{L} d t=3.2 \times 10^{38}$ $\mathrm{cm}^{-2}$ at $500 \mathrm{GeV}$ and $200 \mathrm{GeV}$ respectively. The polarization of both beams is taken as 0.7 .

The statistical error on the two spin asymmetry is estimated as

$$
\delta A=\frac{1}{P_{1} P_{2}} \frac{1}{\sqrt{N}} .
$$

where $P_{1}$ and $P_{2}$ are the beam polarizations and $\mathrm{N}$ is the number of events. Thus, 1600 events are required for $\delta A= \pm 0.05$. On the other hand the one spin asymmetry has an error of

$$
\delta A=\frac{1}{P_{1}} \frac{1}{\sqrt{N}} .
$$

Besides the beam energy the spin configuration (L-type or $\mathrm{N}$-type) is another issue for the running condition. For the time being we leave this question open, and the yield estimates in the following sections are done assuming the above integrated beam time with an appropriate spin setting.

\section{B.2 $W^{+} \rightarrow e^{+}+\nu$}

The production of the intermediate vector boson $W^{ \pm}$of the weak interactions, and its leptonic decay, $W^{+} \rightarrow e^{+}+\nu$, are the classical examples of parity violating processes in hadronic collisions. Yet there is a complete absence of searches for, or measurements of, parity violation at high energy hadron colliders[2]. To quote Maurice Goldhaber (who was quoting astronomers), "The absence of evidence is not the evidence of absence." It is important to measure this production asymmetry. Any discrepancy from the predictions will leads to new physics. In addition, a systematic search for parity violation is proposed. However, it is difficult to estimate rates for this search, since it is totally exploratory, i.e. it is beyond the standard model.

In the context of the standard model, there is one clear parity violating process, $W^{ \pm}$ production, with precise predictions $[3,4]$. Soffer[4] predicts a large single spin asymmetry $A_{L}$, for $W^{+}$, which varies with rapidity, from $20 \%$ at $y_{W^{+}}=-0.3$ to $50 \%$ at $y_{W^{+}}=+0.3$. In PHENIX, the $W^{+}$is detected by a single $e^{+}$in the central spectrometer. The detection of just a single particle allows a surprisingly large acceptance of $13 \%$ of the total $W^{+} \rightarrow e^{+} \nu$ branch[5]. The $\sigma_{W+} \cdot B_{e+\nu}$ is estimated to be $100 p b$ at $\sqrt{s}=500 \mathrm{GeV}$, for a total of $\sim 16000$ detected events from $4 \times 10^{6} \mathrm{sec}$ of integrated beam time. The error should be $\delta A_{L L}^{P V}< \pm 2 \%$. The precise prediction for $A_{L L}^{P V}$ in these conditions is not yet available, but an estimate is twice the average of the single-spin asymmetry over the nominal detector acceptance, or $70 \%$. The energy of the positron is measured precisely in the EM calorimeter, while the charge is determined in the magnetic spectrometer, with $\delta p / p$ of $10 \%$ at $50 \mathrm{GeV} / \mathrm{c}$. There is no other source to create such a high $p_{T}$ electron (except $Z^{0}$ ) so that signal to background should be no problem. A factor of 1000 of charged hadron rejection is all that is required. 


\section{B.3 Direct Photon Production}

This should be a clean measurement of the spin dependent gluon structure function since the dominant subprocess in pp collisions is

$$
g+q \rightarrow \gamma+q \quad,
$$

with $q \bar{q}$ contributing on the order of $10 \%$. The major sources of background for direct photon detection are bremsstrahlung from a jet, and $\pi^{0}$ and $\eta$ decays. These are effectively eliminated, as discussed later, by gamma isolation cuts and $\pi^{0}$ reconstruction. The high segmentation of the PHENIX EM calorimeter, which is driven by the issues of occupancy and energy resolution in the high multiplicity, low $p_{T}$ environment of Heavy Ion Collisions, allows the two gammas from $\pi^{0}$ decay to be resolved for $p_{T}\left(\pi^{0}\right) \leq 25 \mathrm{GeV} / \mathrm{c}[1]$.

\section{B.3.1 Yield estimate of direct photons in PHENIX}

To estimate the yield of direct photons we used the Lund Monte Carlo program PYTHIA which uses the EHLQ set \#1 structure functions. We have considered runs at $\sqrt{s}=200 \mathrm{GeV}$ consistent with the proton-proton runs for the heavy ion physics program, and at $\sqrt{s}=$ $500 \mathrm{GeV}$ to explore a higher $Q^{2}$ region at the highest luminosity.

A fit to the measured direct photon cross sections $[7,8,9,10]$ was carried out by $M$. Beddo et al.[14] using the empirical parametrization;

$$
E \frac{d^{3} \sigma}{d p^{3}}=B \exp \left(-b p_{T}\right) .
$$

This provided the integrated cross sections over the PHENIX acceptance $(-0.35<Y<$ $0.35, \Delta \phi=\pi$ ). These were compared to the PYTHIA results in the $p_{T}$ range of $10-25$ $\mathrm{GeV} / \mathrm{c}$, and are summarized in table B.1 for different transverse momentum bins.

\begin{tabular}{|l|c|r|c|r|r|r|}
\hline Experiment & Reaction & $\sqrt{s}$ & \multicolumn{4}{|c|}{ Cross section $(\mathrm{pb})$ in different momentum bins } \\
& & $\mathrm{GeV}$ & Total $\left(p_{t \gamma}>10 \mathrm{GeV} / \mathrm{c}\right)$ & $10-15$ & $15-20$ & $20-25$ \\
\hline CCOR[7] & $p p$ & 63 & 18.5 & 17.8 & 0.7 & \\
R806[8] & $p p$ & 63 & 8.3 & 8.2 & & \\
PYTHIA & $p p$ & 63 & 8.3 & 8.1 & 0.2 & \\
\hline UA1[9] & $p \bar{p}$ & 630 & 3422 & 2140 & 835 & 298 \\
UA2[10] & $p \bar{p}$ & 630 & 4183 & 2899 & 919 & 266 \\
PYTHIA & $p p$ & 630 & 1838 & 1223 & 401 & 121 \\
\hline PYTHIA & $p p$ & 500 & 1573 & 1076 & 344 & 79 \\
\hline PYTHIA & $p p$ & 200 & 425 & 370 & 43 & 9 \\
\hline
\end{tabular}

Table B.1: Direct photon yield comparison. CERN-ISR and CERN $S p \bar{p} S$ data are compared to the PYTHIA estimates.

PYTHIA seems to underestimate the cross sections at higher energies by about a factor of two. It should be noted that the direct photon cross section is $10-30 \%$ higher in $p \bar{p}$ 
interactions than in $p p[11]$. We will use the PYTHIA predictions for the direct photons yield. The results for the integrated luminosities listed above are shown in Table B.2. The quoted errors are statistical only. Asymmetry errors of less than $4 \%$ and $1 \%$ are achieved at the highest $p_{T}$ bins for 200 and $500 \mathrm{GeV}$ respectively.

\begin{tabular}{|c|r|r|r|r|}
\hline & \multicolumn{2}{|c|}{$\sqrt{s}=200 \mathrm{GeV}$} & \multicolumn{2}{c|}{$\sqrt{s}=500 \mathrm{GeV}$} \\
\hline & Yield & $\Delta A_{L L}$ & Yield & $\Delta A_{L L}$ \\
Total & 136000 & 0.0054 & 1260000 & 0.0018 \\
\hline $10<p_{t \gamma}<15 \mathrm{GeV}$ & 118000 & 0.0058 & 860000 & 0.002 \\
$15<p_{t \gamma}<20 \mathrm{GeV}$ & 13800 & 0.017 & 270000 & 0.004 \\
$20<p_{t \gamma}<25 \mathrm{GeV}$ & 2900 & 0.037 & 63000 & 0.008 \\
\hline
\end{tabular}

Table B.2: Expected number of direct photon events and statistical errors in the asymmetry measurement.

PYTHIA also provides the relative contributions to direct photon production from the various partonic processes; $88 \%$ from the $q g \rightarrow q \gamma$ process and $12 \%$ from the $q \bar{q} \rightarrow g \gamma$ process as shown in Fig B.1. This small contribution from the annihilation channel can be neglected in the analysis of $\Delta G$ ( the gluon helicity distribution) in the measurement of the longitudinal spin asymmetry $A_{L L} .^{1}$

\section{B.3.2 Partonic $x_{b j}$ distributions}

The goal of the $A_{L L}$ measurements is to determine $\Delta G\left(x_{b j}\right)$ when $x_{b j}$ is the momentum fraction that is carried by the parton. The measured asymmetry in direct photon production via the Compton process is written as;

$$
E_{\gamma} \frac{d^{3} \Delta \sigma(p p \rightarrow \gamma X)}{d P_{\gamma}^{3}}=\iint d x_{a} d x_{b} \sum_{f} \Delta q_{f}\left(x_{a}\right) \Delta G\left(x_{b}\right) \frac{d \Delta \sigma}{d t}\left(q_{f} g \rightarrow \gamma q\right),
$$

where $\Delta q_{f}(x)$ and $\Delta G(x)$ are the helicity distributions for quarks and gluons at a given Bjorken $x$, and

$$
\frac{d \Delta \sigma}{d t}=\frac{d \sigma}{d t} \hat{a}_{L L}
$$

is the cross section asymmetry at the parton level.

In inclusive direct photon measurement at fixed $p_{T}$ we determine the integral of $\Delta G\left(x_{\text {gluon }}\right)$ over $x_{\text {quark }}$ weighted by the known quark helicity distributions, $\Delta q$. In principle, detection of the away-side jet is required to determine $x_{b j}$. However,we investigated the accessible regions of $x_{b j}$ for quarks and gluons in direct gamma events these are shown in Figure B.2 with $p_{T \gamma}>10$ and $20 \mathrm{GeV}$ at $\sqrt{s}=200 \mathrm{GeV}$. The distributions of $x_{b j}$ are rather narrow and

\footnotetext{
${ }^{1}$ It should be noted that in the case of transverse spin asymmetry, $A_{N N}$, the contribution from the Compton process vanishes and only the annihilation process contributes to the photon production asymmetry, which relates to the transversity of the quark polarization, $h_{1}(x)$ structure function. An $A_{N N}$ signal from $q \bar{q}$ annihilation is diluted by a factor of 10 by the Compton process.
} 
peak at 0.1 and 0.2 for 10 and $20 \mathrm{GeV}$ photons respectively. This fact ensures that we can derive the $\Delta G(x)$ information by choosing the $p_{T}$ range of the inclusive photons only.

Even though the acceptance for the away side jet is small, a rather modest $p_{T}$ matching between the photon and a jet can be applied to restrict the kinematics of the partons. In Figure B.2 the $x_{b j}$ distributions are also shown for the events which are accompanied with an away side jet. Here the $p_{T}$ and $p_{Z}$ matching between the $\gamma$ and jet are to within $5 \mathrm{GeV} / \mathrm{c}$ which is a rather modest requirement. Though the efficiency to find a away side jet is rather low $(15 \%)$ these events are more sensitive to the particular $x_{b j}$.

\section{B.3.3 Background considerations}

Besides the gluon Compton process and the $q \bar{q}$ annihilation (order of $\alpha_{e m} \alpha_{s}$ ) the next order contribution to the direct photon production is from bremsstrahlung such as $q q \rightarrow q(q \rightarrow \gamma q)$ ( order of $\alpha_{e m} \alpha_{s}^{2}$ ). In the RHIC energy range this process would contribute 20-30\% of the direct photon production [11]. The other background source is the so called "fake" single photons from meson decays $\left(\pi^{0}\right.$ and $\left.\eta^{0}\right)$. In this section we discuss how to handle and eliminate these two kinds of background in PHENIX.

Isolation cut for the same side jet-fragments : By requiring high $p_{T}$ such as $10 \mathrm{GeV} / \mathrm{c}$ for single photon candidates, both of the background sources can only originate from hard QCD scattering and are associated with jet fragments in the same direction, while the "real" direct gamma is only be associated with away-side jet-fragments. This correlation is quite effective for "fake" $\gamma$ and bremsstrahlung $\gamma$ rejection.

The azimuthal distribution of charged particles with respect to direct photon candidates are shown in Figure B.3 for the direct photon events and B.4 for background events. To simulate the background, we have used photons ( mainly from meson decays) with $p_{T}>$ $10 \mathrm{GeV} / \mathrm{c}$ from QCD jets with $p_{T}(j e t)>40 \mathrm{GeV} / \mathrm{c}$. From Figure B.3 and B.4 one can clearly see a difference between the signal and the background.

This azimuthal correlation can be used to estimate the remaining background in the direct photon data sample. The isolation cuts for the single photon candidates appear to effectively eliminate the background. Figure B.5 shows the charged particle multiplicity in a cone of 0.15 radians around a direct photon candidate. A cut on charged particles with $p_{T} \geq 1 \mathrm{GeV} / \mathrm{c}$ eliminates $\sim 85 \%$ of the background with a loss of $\sim 1 \%$ of the signal

$\pi^{0}$ reconstruction capability to reduce "fake" $\gamma$ 's : To estimate the cleanliness of the direct photon candidates after $\pi^{0}$ reconstruction, we estimated the probability of producing "fake" photons from meson decays from a jet with $p_{T}>40 \mathrm{GeV} / \mathrm{c}$ as discussed in the next section in detail. By assuming $100 \%$ reconstruction efficiency when both of decay photons are in the detector and are more than 0.01 radians apart, of all the $\pi^{0}$ 's with $p_{T}$ more than $5 \mathrm{GeV} ; 90 \%$ are reconstructed, $5 \%$ are merged (two photons within 0.01 radians which occurs only when $p_{T}\left(\pi^{0}\right)$ is greater than $25 \mathrm{GeV} / \mathrm{c}$ ) and the remaining $5 \%$ are "fake" due to asymmetric decays where one gamma is out of the acceptance. Most of those "merged" gammas can be recognized as two gammas from shower size analysis even though they can not be reconstructed as mesons. Even though the two-gamma reconstruction efficiency is 
degraded from $100 \%$ to $90 \%$ due to, for example, hadronic shower overlap, the rejection power of the reconstruction will remain at $\sim 85 \%$.

By applying both of these rejection methods, the purity of direct photon candidates will be excellent. For the worst case such as $\gamma_{\text {real }} / \pi$ being $0.1, \gamma_{\text {fake }} / \gamma_{\text {real }}$ will be $\sim 1$ after the $\pi^{0}$ detection. The isolation cut will bring $\gamma_{\text {fake }} / \gamma_{\text {real }}$ down to $\sim 0.15$. For the bremsstrahlung gamma ( $20 \%$ to $30 \%$ of the signal), $\gamma_{\text {brems }} / \gamma_{\text {real }}$ will be $\sim 0.05$ after the isolation cut.

\section{B.4 $\pi^{0}$ production}

The inclusive $\pi^{0}$ polarization asymmetry can be measured with reconstructed $\pi^{0} \rightarrow \gamma+\gamma$ in the range $10 \leq p_{T} \leq 25 \mathrm{GeV} / \mathrm{c}$. In this $p_{T}$ range most $\pi^{0} \mathrm{~s}$ are produced from QCD jets. Compared to other jet fragments $\pi^{0}$ triggering should be quite simple in the PHENIX apparatus.

At the parton level, high $p_{T} \pi^{0}$ s originate from several subprocesses. Major channels are;

- $q q \rightarrow q q(\sim 20 \%)$,

- $q g \rightarrow q g(\sim 50 \%)$ and

- $g g \rightarrow g g(\sim 30 \%)$.

Due to this mixture, the theoretical prediction for the production asymmetry becomes difficult. However, the higher yield of $\pi^{0}\left(\gamma / \pi^{0} \simeq 1 / 4-1 / 10\right.$ at the upper $p_{T}$ range) results in an asymmetry error, $\delta A \leq \pm 1 \%$, thus $\pi^{0}$ measurements could be used to explore other spin phenomena;

- Parton helicity distributions: Theoretical predictions for $A_{L L}\left(\pi^{0}\right)$ show notable differences when gluons are polarized [12].

- Spin transversity measurements using spin transfer: Correlations between the transversely polarized beam axis to the plane of two produced $\pi^{0} \mathrm{~s}$, may yield some information on quark transversity structure functions [13].

- Higher order QCD tests: Single spin asymmetry, $A_{N}$, could well be related to twist-3 effects.

- Parity violation in hadron interactions at high energy: The interference between strong and weak interactions may be large enough to see $\left(10^{-4}-10^{-2}\right)$ at RHIC energies. However, a high degree of control over the systematics is required for such a measurement.

The recent data from FNAL-E704 showed large single transverse spin asymmetry $A_{N}$, of more than $10 \%$, at large $p_{T}(\sim 3 \mathrm{GeV})$ in $p \uparrow p \rightarrow \pi^{0}+X$ reactions at $\sqrt{s}=20 \mathrm{GeV}$ [17]. This asymmetry could originate from higher twist effects in QCD as discussed in the section A. It would be quite interesting to repeat this type of measurements at RHIC at high $Q^{2}$. The FNAL-E704 results generated considerable theoretical interest that may lead to more precise predictions to be tested by the time RHIC comes on line.

In the following subsection we discuss the feasibility of $\pi^{0}$ detection in PHENIX in the range of $10<p_{T}<25 \mathrm{GeV} / \mathrm{c}$. Above $25 \mathrm{GeV} / \mathrm{c}, \pi^{0}$ can be measured using single clusters, but the large direct photon background may make the results difficult to interpret cleanly. 


\section{B.4.1 $\pi^{0}$ reconstruction}

The energy and position resolution which PHENIX offers will provide good $\pi^{0}$ mass resolution as shown in Fig. B.6. Here $7.1 \% / \sqrt{E}+0.7 \%$ is assumed for the energy resolution and $7 \mathrm{~mm} / \sqrt{E}+0.8 \mathrm{~mm}$ is assumed for the position resolution as quoted in the PHENIX pCDR. The background below the pion mass is relatively small especially in the high $p_{T}$ region.

The efficiency of $\pi^{0}$ reconstruction is important both in $\pi^{0}$ physics as well as in direct photon physics. The inefficiency will be caused by;

1. merging of two gamma into one,

2. asymmetric decay of $\pi^{0}$ ( one gamma out of the acceptance) and

3. accidental shower overlap.

Minimum angle between gamma pairs produced through $\pi^{0}$ decay is given by

$$
\Delta \theta_{l a b}=2 \frac{m_{\pi^{0}}}{E_{\pi^{0}}}
$$

where $m$ and $E$ are the mass and energy of the $\pi^{0}$ respectively. Figure B.7 shows the opening angle distribution of two gammas from $\pi^{0}$ decays. To ensure the good reconstruction efficiency up to $25 \mathrm{GeV} / \mathrm{c}$ of $p_{T}$, a two gamma separation of 0.01 radians is required. This is attainable with the Standard PHENIX segmentation $\left(5 \times 5 \mathrm{~cm}^{2}\right)$ of the EM calorimeter.

In Figure B.8 the $p_{T}$ distribution of $\pi^{0}$ from QCD hard scattering with $p_{T}(j e t) \geq 40 \mathrm{GeV} / \mathrm{c}$ is shown when both of the decay gammas are in the acceptance, also shown are the rates of two $\gamma$ 's merging. Significant merging occurs above $25 \mathrm{GeV} / \mathrm{c}$, below which the accidental merging with another gamma (not a partner) occurs at a level of $5 \%$. For comparison the merging situation with 0.02 radians separation is also shown, which limits the $p_{T}$ range of $\pi^{0}$ to $\sim 12 \mathrm{GeV} / \mathrm{c}$, and below that the accidental merging rate is $\sim 20 \%$.

The asymmetric decay of $\pi^{0}$ and $\eta$ is a typical source of fake direct photons by missing one of the decay photons out of the acceptance. With a simple geometrical Monte Carlo calculation the rate of fake gammas is found to be $2-4 \%$ of $\pi^{0}$ in the $p_{T}$ range of interest as shown in Fig.B.9[14]. This causes an inefficiency in $\pi^{0}$ reconstruction but is easily correctable.

\section{B.4.2 Triggering}

As described in pCDR, we have in the scope "local $\Sigma p_{T}$ trigger" in the EM calorimeter, which provides perfect triggers for $W^{ \pm}$, direct $\gamma$, and $\pi^{0}$ at hight $p_{T}$. The rate will not be a problem for such high $p_{T}$ events.

\section{B.5 Dilepton Production}

\section{B.5.1 Drell Yan}

Drell-Yan production is yet another probe of both the sea quark as well as the gluon structure functions, through the two dominant processes $q \bar{q} \rightarrow \gamma^{*} \rightarrow l^{+} l^{-}$and $g q \rightarrow \gamma^{*} q\left(\gamma^{*} \rightarrow l^{+} l^{-}\right)$ respectively. We use the PYTHIA program to study muon pair production in PHENIX. Figure $\mathrm{B} .10$ is one representative plot showing the relative contribution of the various partonic processes for the di-muon mass range $2-3 G e V / c^{2}$. Several points are worth noting: 
1. A $p_{t}$ cut of $1 \mathrm{GeV} / \mathrm{c}$ separates the production into regions where either one of the processes $q \bar{q}$ and $g q$ dominates.

2. The $q \vec{q}$ events with $p_{t} \leq 1 \mathrm{GeV} / \mathrm{c}$ are quite clean. These show little or no contamination from accompanying bremsstrahlung which could only mask the direct access to partonic spin information (see $q \bar{q}(\mathrm{NB})$ in Fig. B.10)

3. The rate of the $g q$ process is $10 \%$ of the $q \bar{q}$ process.

A similar $p_{T}$ cut could be applied to distributions at higher dilepton masses.

The sensitivity of the Drell Yan process to the parton spin structure functions is enhanced if the participant partons were at relatively large $x_{b j}\left(x_{b j}>0.1\right)$ since it is generally believed that at $x_{b j}=0, \Delta q / q$ and $\Delta \bar{q} / \bar{q}$ are zero. The $x_{b j}$ correlations for the PHENIX acceptance are shown in Figure B.11 and B.12. for the dilepton mass of 2-3 GeV/c $c^{2}$ and $12-15 \mathrm{GeV} / \mathrm{c}^{2}$. The subprocess $q g \rightarrow \gamma^{*} q$ shows both $x_{b j}$ 's to be sizable. However but for $q \widetilde{q} \rightarrow \gamma^{*}$ one $x_{b j}(\bar{q})$ is small $\left(10^{-2}\right)$ so that a small analyzing power is expected for the two spin asymmetry from the annihilation channel. This also suggests that we can measure the gluon helicity distribution via $A_{L L}$ cleanly ( note that the requirement of high $p_{T}$ for muon pairs is another tool to eliminate the annihilation channel). This would represent another independent experiment to augment the $A_{L L}$ measurements of $\Delta G$ measurements in the direct photon channel. The error in the asymmetry measurement is expected to be $\Delta A_{L L} \sim 2 \%$.

With transversely polarized beams the new field of transverse spin effects can be explored in the muon-pair channel by measuring the correlation of the plane of the muon-pair to the spin axis. This provides another approach to quark transversity, $h_{1}$. The PHENIX endcap muon detector is ideal for such measurements since it covers the full azimuth over the rapidity range $1.1 \leq y_{\mu^{+} \mu^{-}} \leq 2.5$. The estimated event rates[6] are given in the Table below for $4 \times 10^{6}$ sec running time at $\sqrt{s}=200$ and $500 \mathrm{GeV}$.

$\begin{array}{ccc}m_{\mu^{+} \mu^{-}} \mathrm{GeV} & \text { Events at } 200 \mathrm{GeV} & \text { Events at } 500 \mathrm{GeV} \\ 2 \text { to } 5 & 96,000 & 240,000 \\ 5 \text { to } 9 & 23,000 & 79,000 \\ 9 \text { to } 12 & 4,000 & 19,000\end{array}$

Table B.3: Di-Muon rates for PHENIX Endcap and Barrel.

The possibility of muon measurements in both the endcap and the barrel enhances the accessibility to large partonic $x_{b j}$. It will be relatively easy to trigger and identify muons in the PHENIX endcap. Monte Carlo studies suggest that triggering on the total number of hits and the deepest plane hit, will reject more than $95 \%$ of pions with about a loss of $10 \%$ of the muons in the energy range of $1-10 \mathrm{GeV}$. The case in the central region is not as easy since there is no active muon identification. Here, Monte Carlo studies of pion spectra and their decays into $\mu \nu$ show a very sharp drop in rate above $p_{T} 2.5-3 \mathrm{GeV} / \mathrm{c}$. Muons will be identified by tracking and requiring a minimum ionization signal in both the TRD and the EM calorimeter. 


\section{B.5.2 $J / \Psi$ and $\chi_{2}$ production}

Although so many years have passed after the first discovery of $J / \psi$ and many charmonium experiments have been performed, the mechanism of charmonium production in hadronic interaction remains somewhat unresolved. For example, it is surprising that there is no experimental agreement on what fraction of $J / \psi$ is via $\chi$. The study of charmonium production with polarized beams could provide further information on this subject, and could serve as another channel to measure the gluon polarization [18].

The hadronic production of the charmonium states involves three kinds of parton diagrams: gluon fusion, light quark annihilation, and color evaporation. At higher energies,
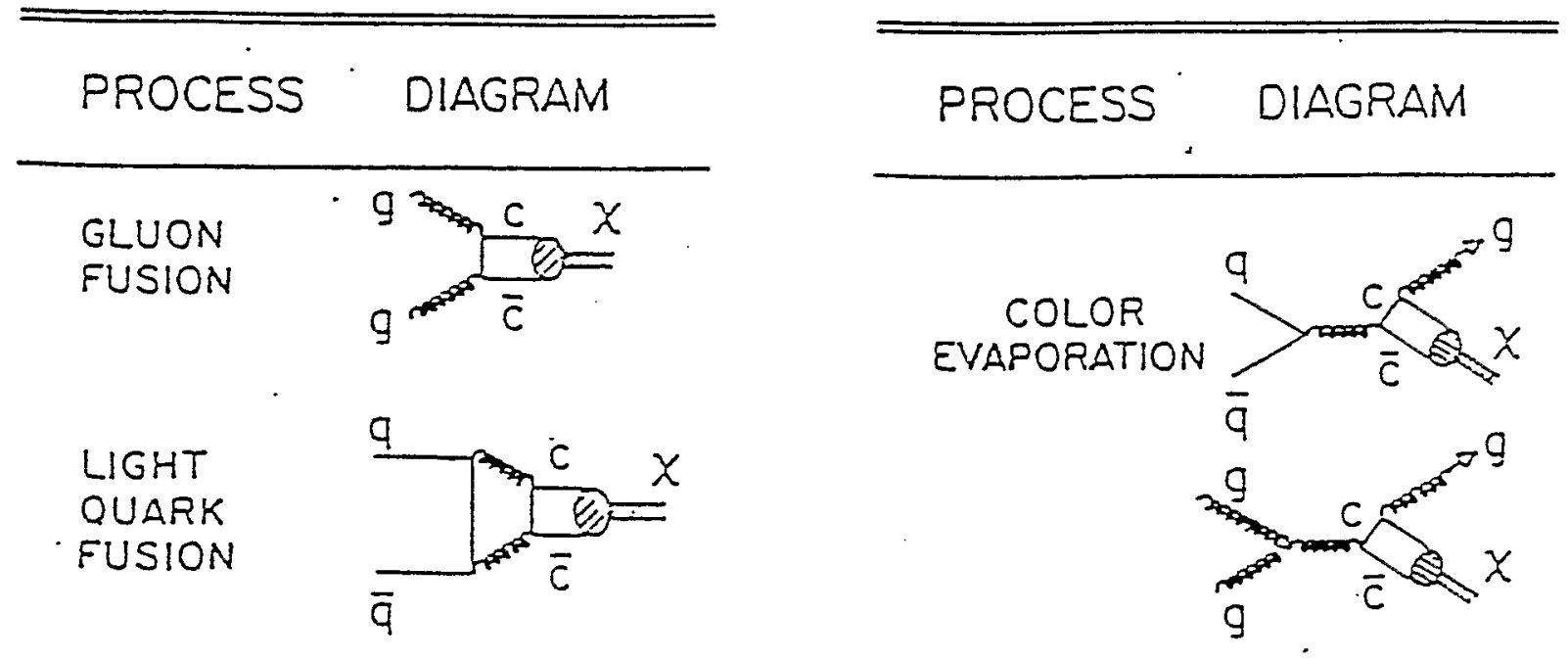

processes like color evaporation will play an important role in charmonium production especially in the high $p_{T}$ region. The dominant mechanisms are $g g \rightarrow(c \bar{c}) g, g q \rightarrow(c \bar{c}) q($ or $\bar{q})$ and $q \bar{q} \rightarrow(c \bar{c}) g$. Here $(c \bar{c})$ is either any of the S states $(\psi)$ or the $\mathrm{P}$ states $(\chi)$. Theoretical predictions for the double spin asymmetry $A_{L L}$ as a function of $p_{T}$ by R. Robinett[18] are shown in Fig.B.13 Two polarized gluon distributions are compared; one using the standard set[23] and the other set with large $\Delta G$ by Z. Kunszt[24] which explains the EMC data. At $\sqrt{s}=200 \mathrm{GeV}$ using $B \cdot d \sigma / d y=10^{-32} \mathrm{~cm}^{2}$, we expect $200 \mathrm{~K} J / \psi \rightarrow e^{+} e^{-}$and over $1 \mathrm{M}$ $J / \psi \rightarrow \mu^{+} \mu^{-}$in the PHENIX barrel and endcap spectrometers which will allow a systematic study of the $p_{T}$ dependence of $A_{L L}(J / \psi)$. We expect $\Delta A_{L L} \sim 2 \%$ for the region of $p_{T}>3 \mathrm{GeV} / \mathrm{c}$, which is sensitive to the large gluon polarization.

Most of the $J / \psi^{\prime}$ s in the barrel $\left(e^{+} e^{-}\right)$are at low $p_{T}$, where it is generally believed the $\chi$ states are mainly produced by gluon-gluon fusion. In such a model, $\chi_{0}$ and $\chi_{2}$ can be produced directly while $\chi_{I}$ is not. Note that $J / \psi$ can not be produced directly by either gluon fusion or by light quark fusion due to $\mathrm{C}$ invariance. Since the branching ratio of $\chi_{0} \rightarrow J / \psi+\gamma$ is small $\left(6 \times 10^{-3}\right)$, the $J / \psi+\gamma$ channel in proton proton collisions should result from $\chi_{2}$ decays only (no $\chi_{1}$ ), which is supported experimentally[22] although with 
limited statistics. If all $J / \psi \rightarrow e^{+} e^{-}$'s are produced via $\chi, 2,000 \chi$ 's will be detected with a gamma in the high resolution CsI EM calorimeter which is capable of separating the $\chi_{1}$ and the $\chi_{2}$ mass peaks. Absence of $\chi_{1}$ will prove the gluon fusion dominance. Using the medium resolution calorimeter also, $8,000 \chi$ 's will be obtained, with which we can study the source of $J / \psi$ production.

In the gluon fusion process $M_{\chi_{2}}^{2}=x_{1} \times x_{2} \times s$ and $x_{F}\left(\chi_{2}\right)=x_{1}-x_{2}$, where $x_{1}$ and $x_{2}$ are the longitudinal-momentum fraction of gluons. Thus at $\sqrt{s}=200 \mathrm{GeV}$ a small asymmetry is expected since $x_{1}$ and $x_{2}$ are small. This leads to a future possibility to run RHIC with smaller $\sqrt{s}, 50-100 \mathrm{GeV}$. Theoretical estimates are done by Doncheski and Robinett[18] using $\Delta G / G$ by Z. Kunszt[24]. The predictions are shown in Fig.B.14 for $\chi_{2}$ and $J / \psi$. In both cases the predicted asymmetries are sizable (more than $10 \%$ ) at $\sqrt{s}=50 \mathrm{GeV}$.

\section{B.6 Schedule and Request}

The scope of this section $[\mathrm{B}]$ is based on the standard version of the PHENIX detector. We envisage no major additions or changes in either the detector setup or trigger system. We assume that the heavy ion run will preceed the first run with polarized protons, thus the detector will have been tried and tested. Running at $\sqrt{s}=200 \mathrm{GeV}$ could be a part of the normal heavy ion running schedule with protons. The $\sqrt{s}=500 \mathrm{GeV}$ will be separate.

The only issue is the segmentation of the EM calorimeter. For the spin physics we would like to have $5 \times 5 \mathrm{~cm}^{2}$ from beginning. If we must start with the $10 \times 10 \mathrm{~cm}^{2}$ option due to the budget limitation the physics capability for spin will be significantly reduced. We will still be able to start with $1 / 4$ of calorimeter which consists of lead glass with a fine segmentation, with some lack of systematic check such as left-right consistency. 


\section{References}

[1] PHENIX, Preliminary Conceptual Design Report, June 26, 1992.

[2] See, however, T. Goldman and D. Preston, Phys. Lett. 168B, 415 (1986), and references therein.

[3] F. E. Paige, T. L. Trueman and T. N. Tudron, Phys. Rev. D19, 935 (1979). See also G. Bunce, et al. DPF, Snowmass 1982, p 489 (BNL-32150); F. E. Paige and M. J. Tannenbaum, BNL-33119 (1983).

[4] Jacques Soffer, private communication.

[5] Vladimir Rykov, M. J. Tannenbaum, internal report to RSC, July 14, 1992.

[6] Vladimir Rykov, S. Heppelmann, internal report to RSC, July 14, 1992.

[7] A. L. S.Angelis et al. Phys. Lett. 94B, 106 (1980). (CCOR-R110 Collaboration)

[8] E. Anassontzis et al. Z. Phys. C13, 277 (1982). (ISR-R806 Collaboration)

[9] UA1 Collaboration, C. Albajar, Phys. Lett. 209B, 358 (1988).

[10] UA2 Collaboration, R. Ansari, Phys. Lett. 176B, 239 (1986).

[11] E. L. Berger et al. Nucl. Phys. B239, 52 (1984).

[12] G.Ramsey and D. Sivers, ANL-HEP-PR-90-76. D. L. Adams et al. Phys. Lett. 261B 197 (1991).

[13] J. Collins, private communication.

[14] M. E. Beddo, H. Spinka and D. G. Underwood, ANLHEP internal report, June 25, 1992.

[15] UA2 Collaboration, J. Alitti et al, Physics Letters B263, 544 (1991); see also Eric Lancon, Thesis, Saclay report CEA-N-2549 (1988).

[16] UA1 Collaboration, C. Albajar, et al, Nucl. Phys B335, 2611990.

[17] A. Yokosawa, in Proceedings of Polarized Collider Workshop, 1990.

[18] R. Robinett, Phys. Rev. D43, 113 (1991); M. A. Doncheski and R. W. Robinett, Phys. Lett. B248, 188 (1990).

[19] V. Barger and A. D. Martin, Phys. Rev. D31 1051 (1985).

[20] For instance, see A. L. S. Angelis, et al, Nucl. Phys. B209, 284 (1982).

[21] For instance, see A. L. S. Angelis, et al, Phys. Lett. 87B, 398, 1979.

[22] D. A. Bauer et al., Phys. Rev. Lett. 54, 753 (1985). 
[23] R. Baier et al., Z.Phys. C19, 251 (1983).

[24] Z. Kunszt, Phys. Lett. B218, 243 (1989). 


\title{
Figure Captions
}

\author{
Figure B.1:
}

The $p_{T}$ distribution of direct $\gamma$ 's at $\sqrt{s}=200 \mathrm{GeV} / \mathrm{c}$. The hatched area is the contribution from $q \bar{q} \rightarrow \gamma g$.

Figure B.2:

The $x_{b j}$ distributions of $\mathrm{u}$-quarks and gluons for direct photons detected in PHENIX with $p_{T \gamma}>10 \mathrm{GeV} / c$ (upper) and $p_{T_{\gamma}}>20 \mathrm{GeV} / c$ (lower) at $\sqrt{s}=200 \mathrm{GeV}$. The hatched areas are those with away-side jet detection (see text).

Figure B.3:

Azimuthal distributions of charged particles with respect to direct photons detected in PHENIX. Curves are for all charged particles (solid), charged particles in the PHENIX acceptance (dashed), and detected charged particles with $p_{T}>1.0 \mathrm{GeV}$ (hatched).

Figure B.4:

Azimuthal distributions of charged particles with respect to the photons detected in PHENIX. Curves are for all charged particles (solid), charged particles in the PHENIX acceptance (dashed), and detected charged particles with $p_{T}>1.0 \mathrm{GeV}$ (hatched).

Figure B.5:

Multiplicity distributions of charged particles within a 0.15 radians cone around a "real" direct photon and similarly "fake" direct photon.

Figure B.6:

Invariant mass distributions of two gammas from hard QCD scattering with $p_{T}(j e t)=$ $40 \mathrm{GeV} / \mathrm{c}$.

Figure B.7:

Opening angle distributions of two gammas from $\pi^{0}$ decays with $p_{T}$ of $10,15,20,25$ and 30 $\mathrm{GeV} / \mathrm{c}$

Figure B.8:

Transverse momentum distributions of $\pi^{0}$ 's in the PHENIX acceptance from QCD hard scattering with $p_{T}(j e t) \geq 40 \mathrm{GeV} / \mathrm{c}$. The hatched area represents $\pi^{0}$ 's with accidental overlap with a gamma from other decays, and the blackened area is for those merging with a partner gamma $(\Delta \theta=0.01)$. The dashed line represents both cases where gammas merge with $\Delta \theta=0.02$.

Figure B.9:

The probability of having a "fake" gamma from $\pi^{0}$ decays within the geometrical acceptance as a function of $p_{T}$. 


\section{Figure B.10:}

The $p_{T}$ distributions of muon pairs in PHENIX with mass of $2-3 \mathrm{GeV} / \mathrm{c}^{2}$ at $\sqrt{s}=200 \mathrm{GeV}$. Contributions from each subprocess are shown. "NB" stands for "No bremsstrahlung" meaning a $q \bar{q}$ annihilation to $\gamma^{*}$ without bremsstrahlung.

\section{Figure B.11:}

The correlation of $x_{b j}$ 's of partons for muon pairs detected in PHENIX with the mass range of 2-3 GeV/ $\mathrm{c}^{2}$. For all, $q \bar{q}, q \bar{q}$ without bremsstrahlung, and $g q$ subprocesses.

Figure B.12:

The correlation of $x_{b j}$ 's of partons for muon pairs detected in PHENIX with the mass range of $12-15 \mathrm{GeV} / \mathrm{c}^{2}$. For all, $q \bar{q}, q \bar{q}$ without bremsstrahlung, and $g q$ subprocesses.

\section{Figure B.13:}

The double spin asymmetry $A_{L L}$ as a function of $p_{T}$ for charm production in pp collisions at several different values of $\sqrt{s}$. The polarized gluon distribution is taken as the "standard $\Delta G[23](\mathrm{a})$ and the large $\Delta G[24](\mathrm{b})$.

Figure B.14:

Predictions for $-A_{L L}$ in $\chi_{2}$ production ( a ) and $J / \psi$ production ( $\mathrm{b}$ ) as a function of $M_{\chi_{2}} / \sqrt{s}$, from Ref.[18]. 


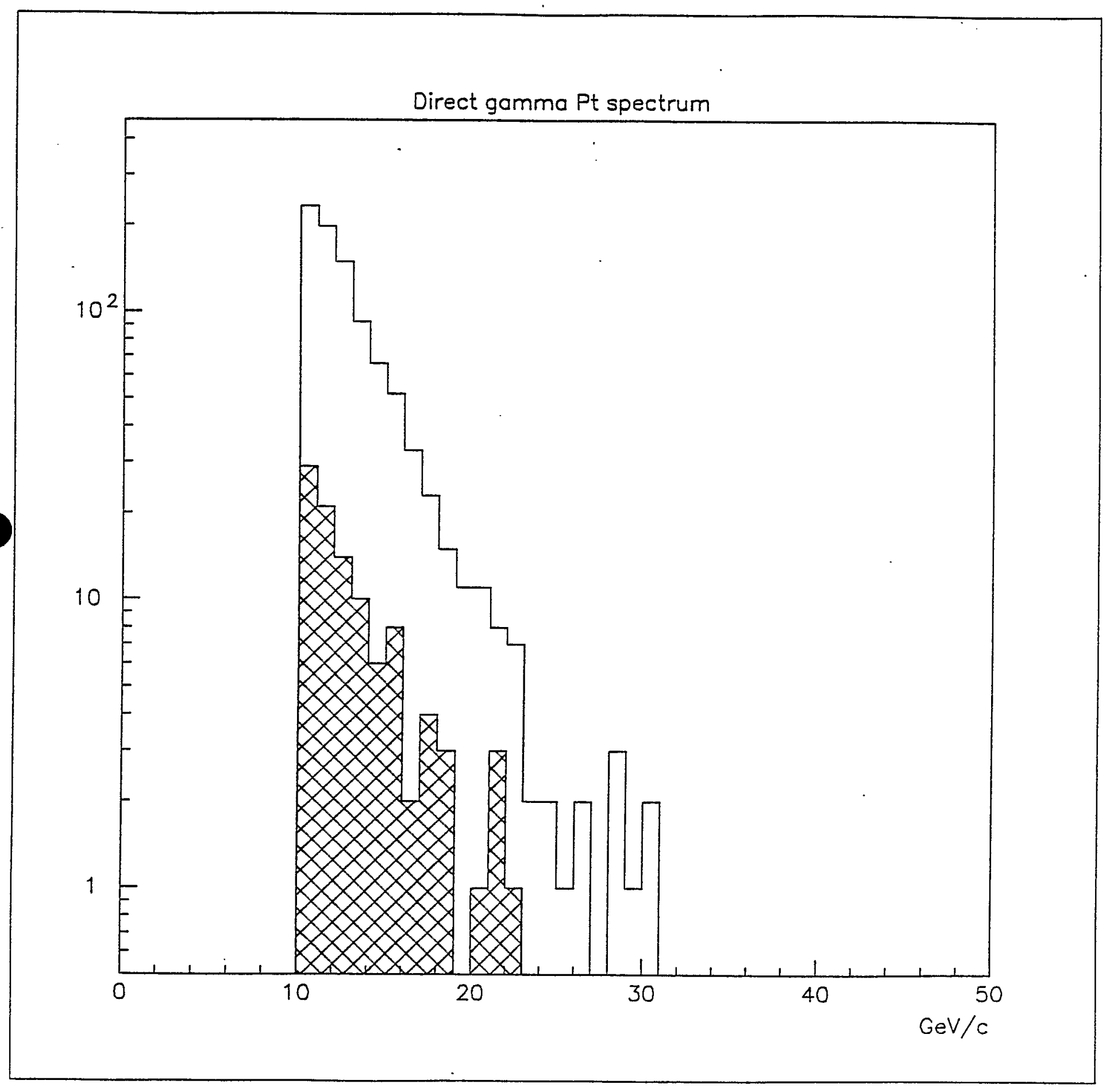

Figure B-1 
Xbj distribution
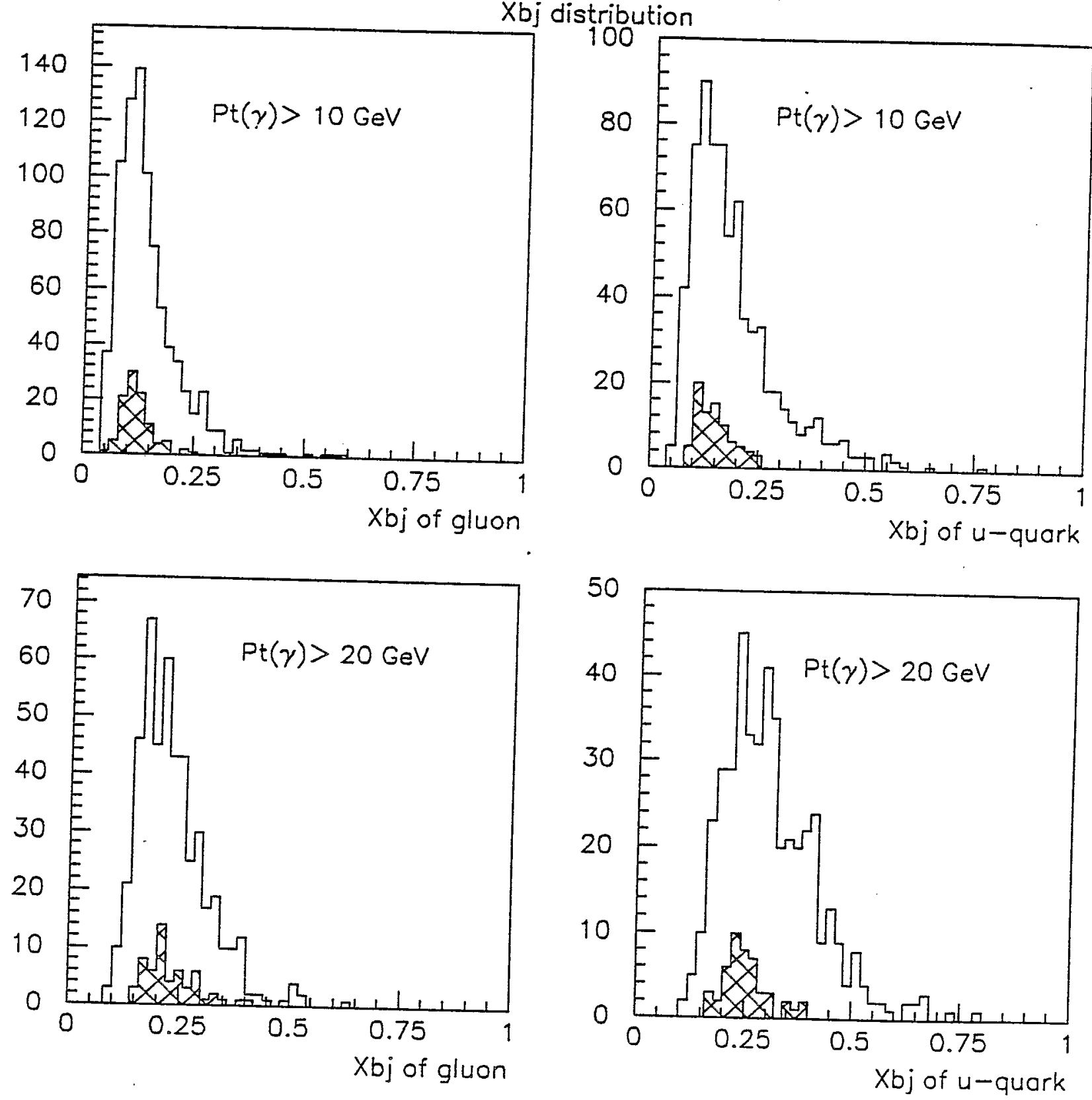


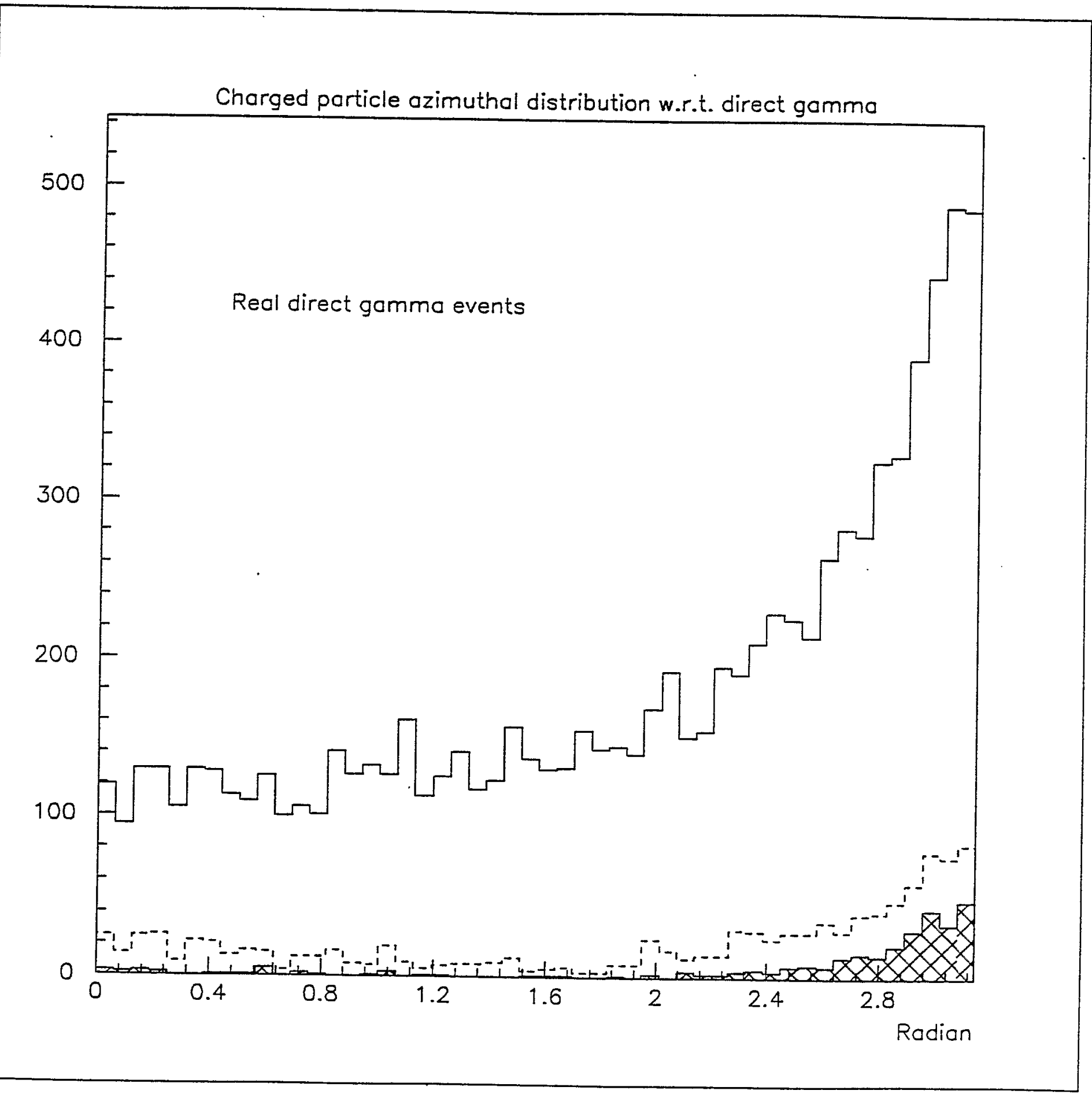

Figure B-3 
.

Charged particle azimuthal distribution w.r.t. direct gamma

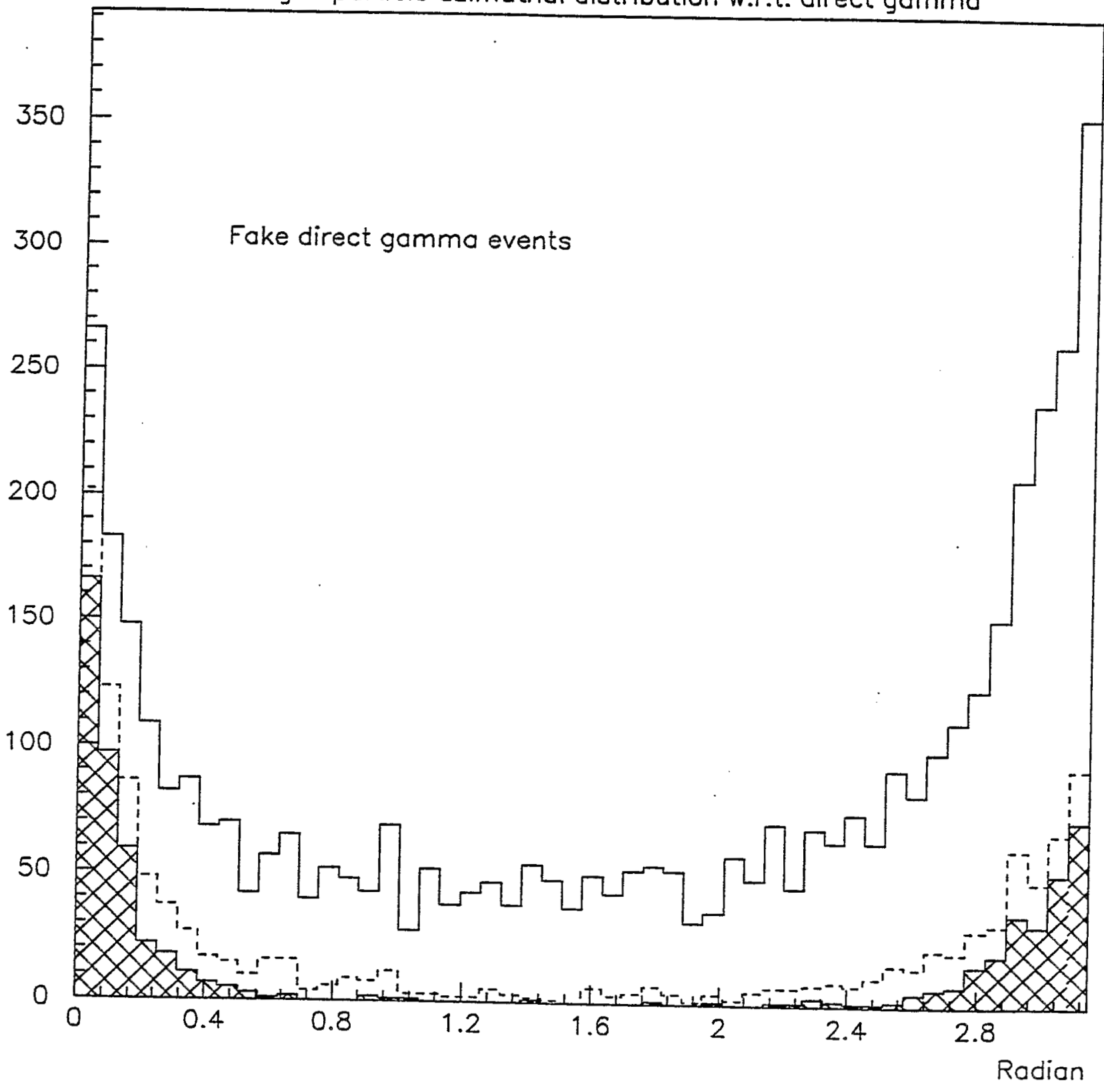

Figure $B-4$ 
Charged particle multiplicity distribution
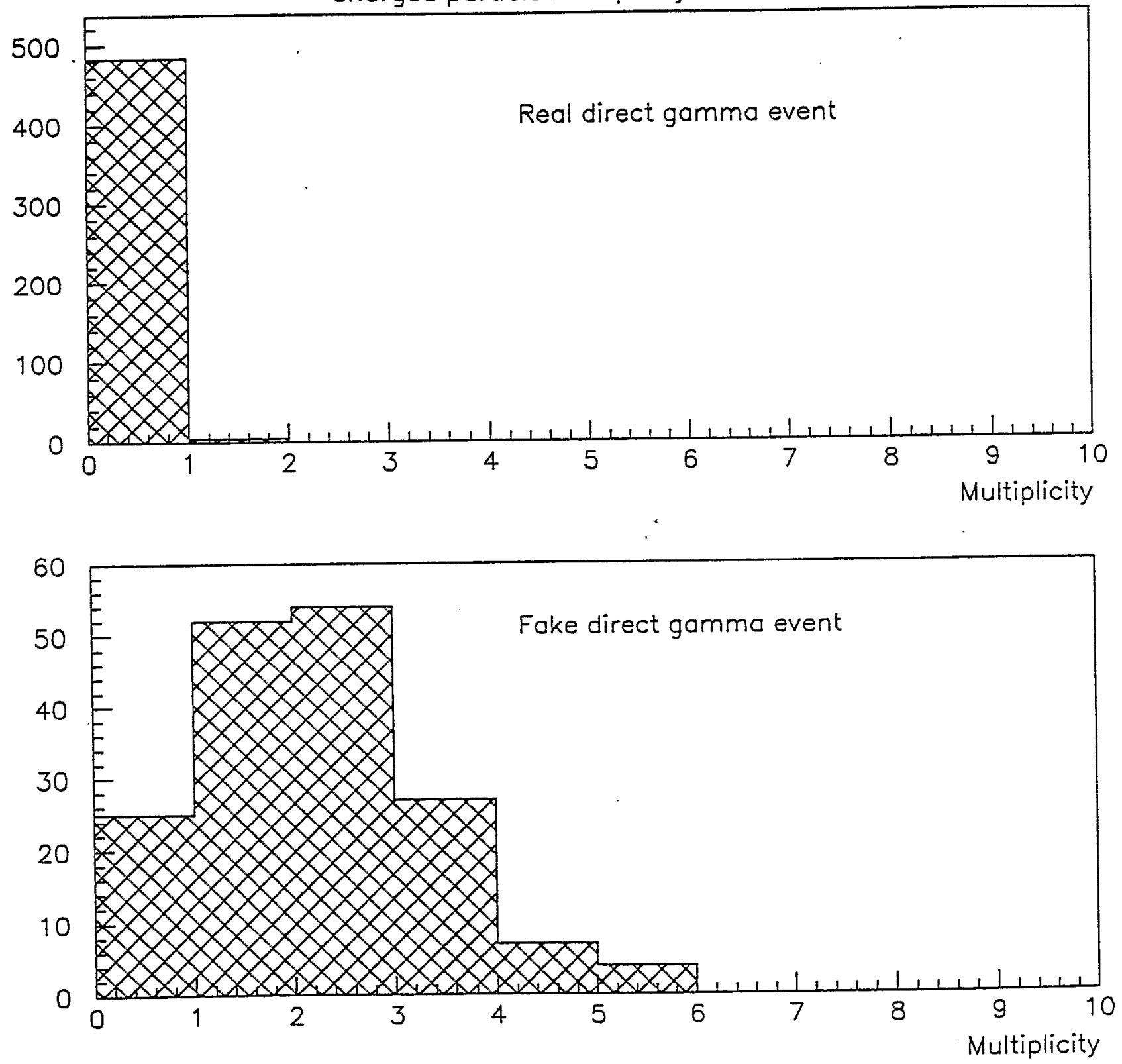


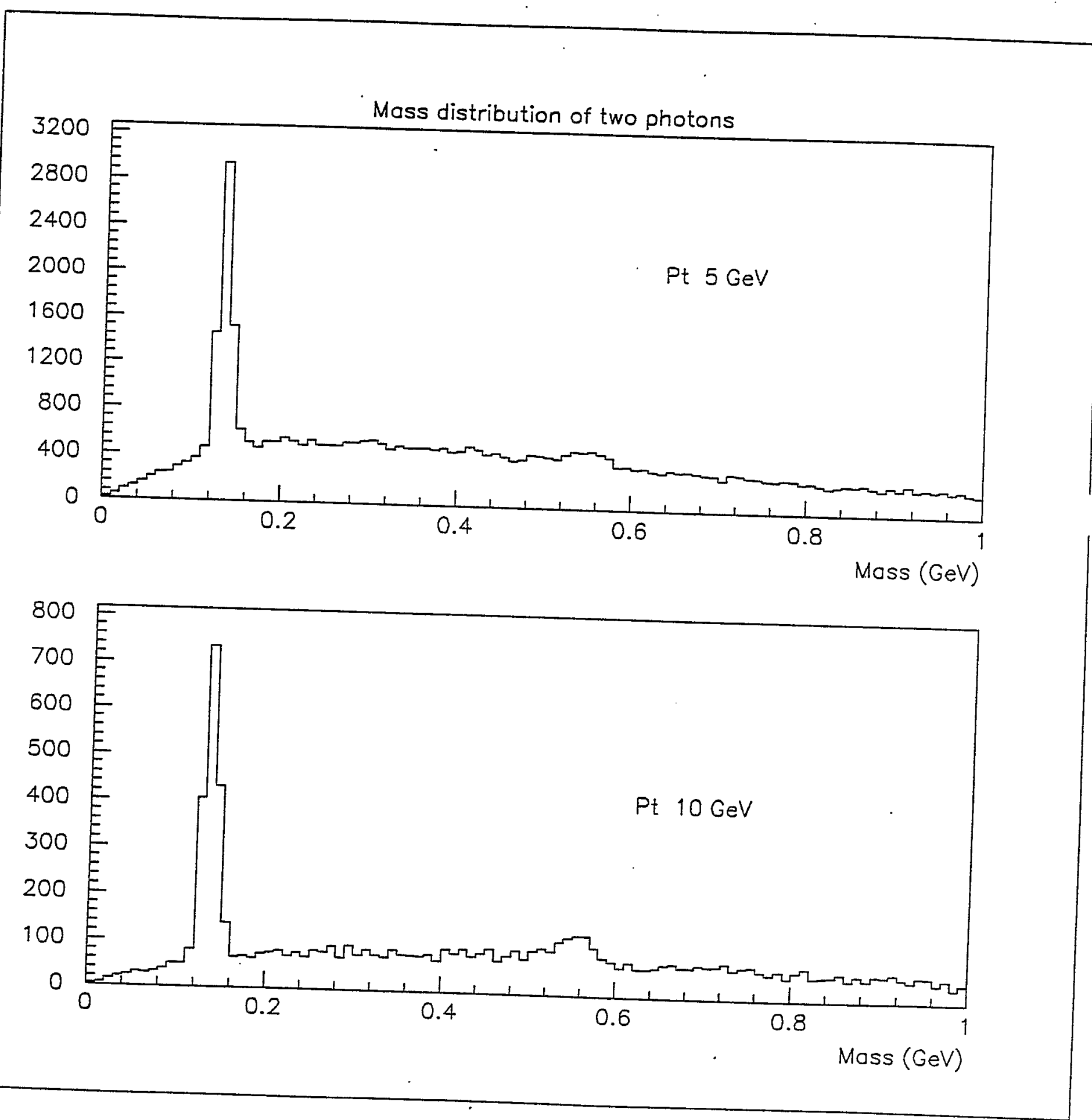

Figure B-6 
2000

0

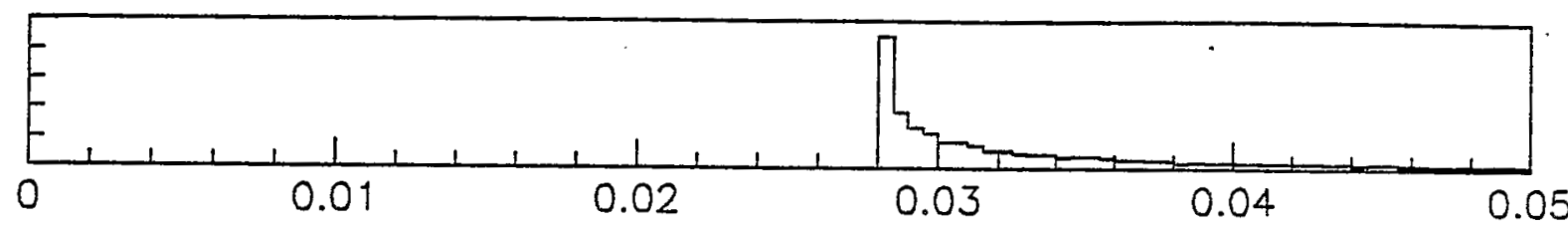

$\mathrm{Pt}=10 \mathrm{GeV}$ piO seperation

2000

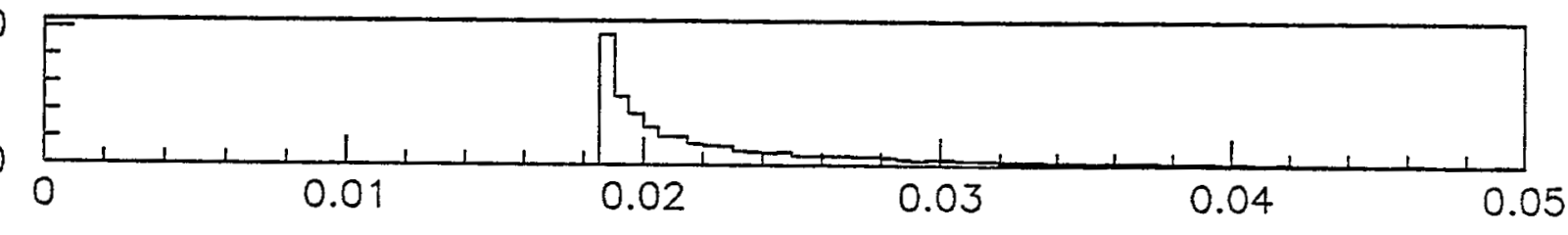

$P t=15 \mathrm{GeV}$ pio seperotion

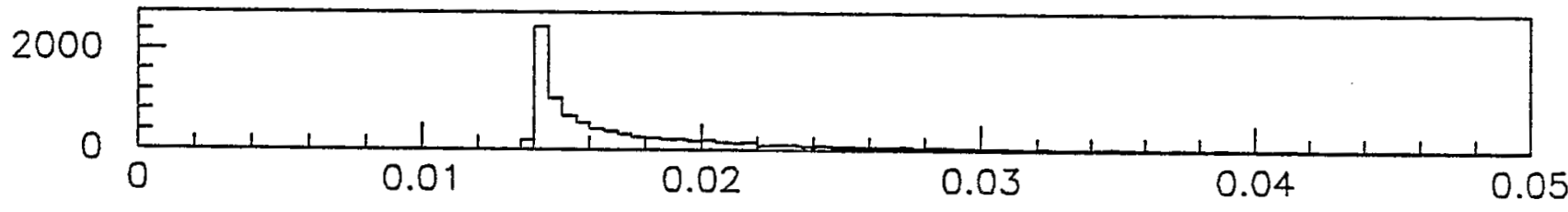

$\mathrm{Pt}=20 \mathrm{GeV}$ piO seperation

2000

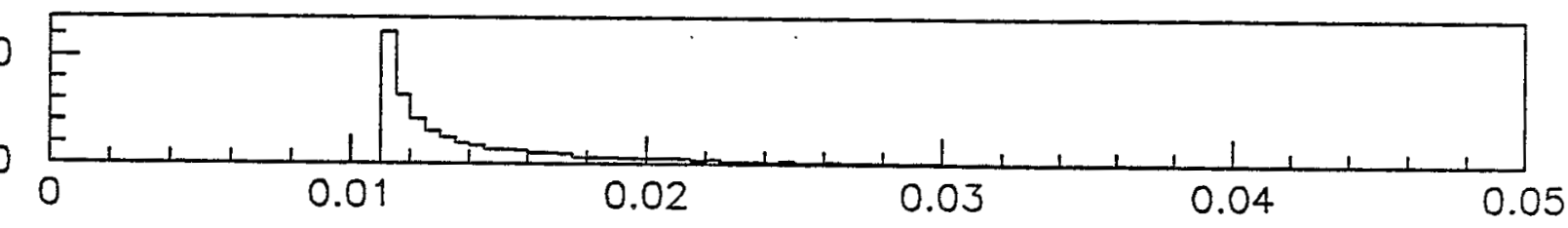

$\mathrm{Pt}=25 \mathrm{GeV}$ piO seperation

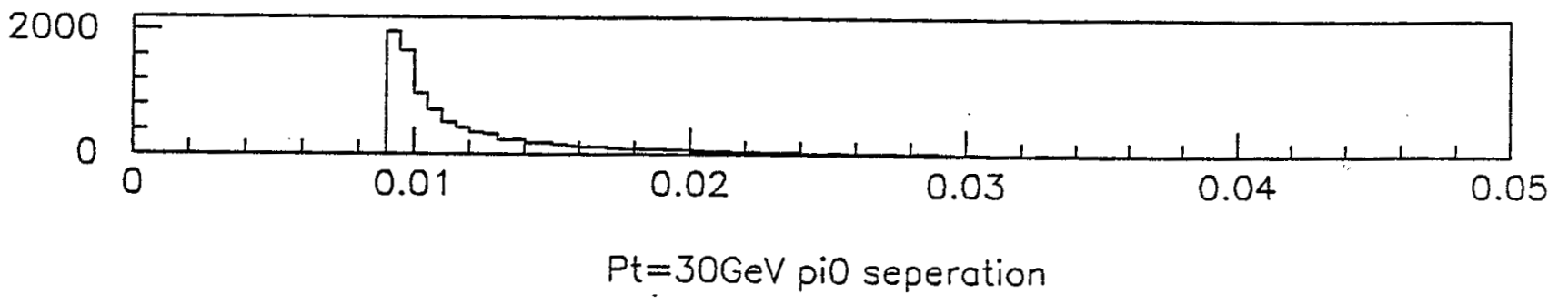




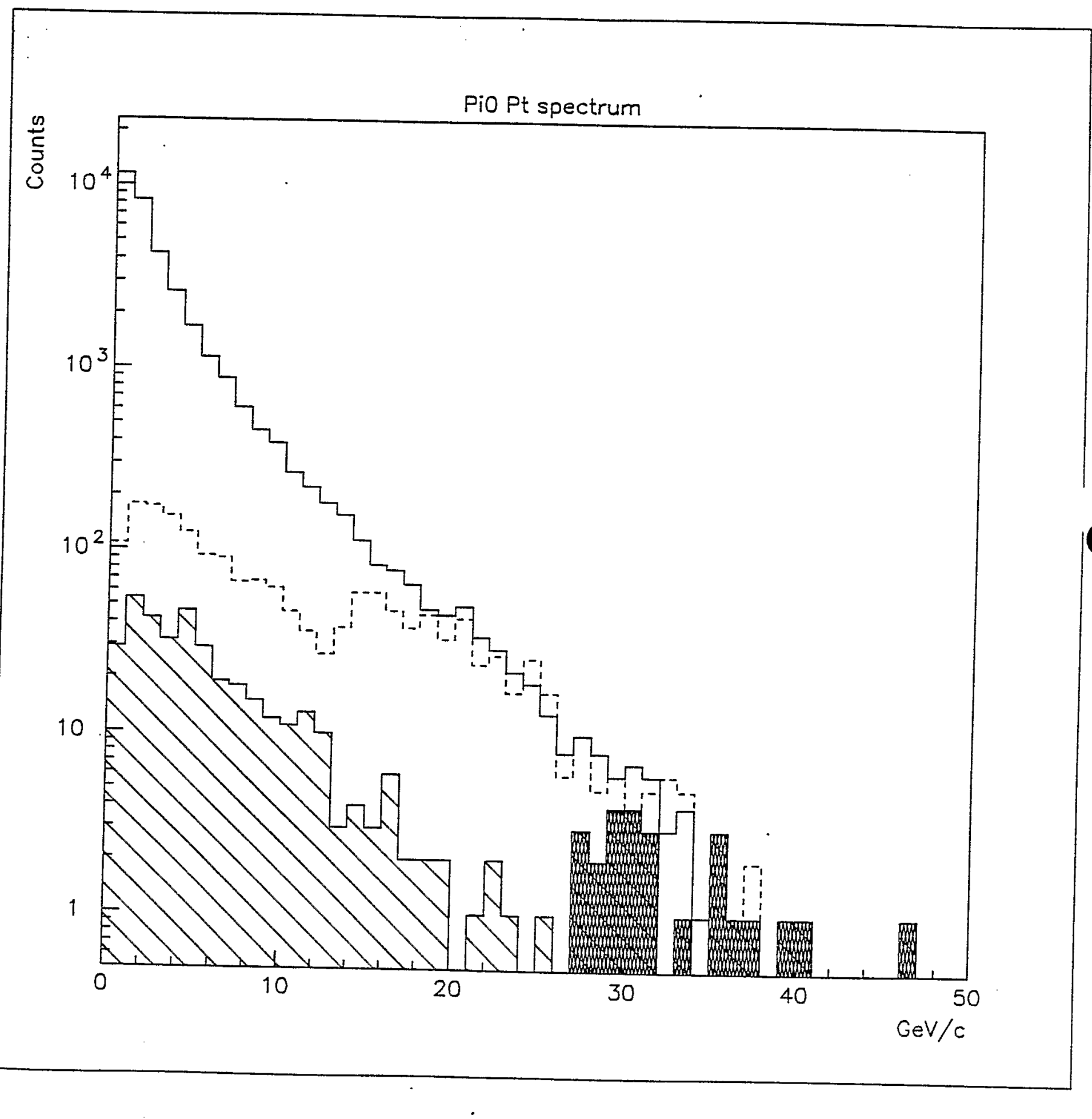




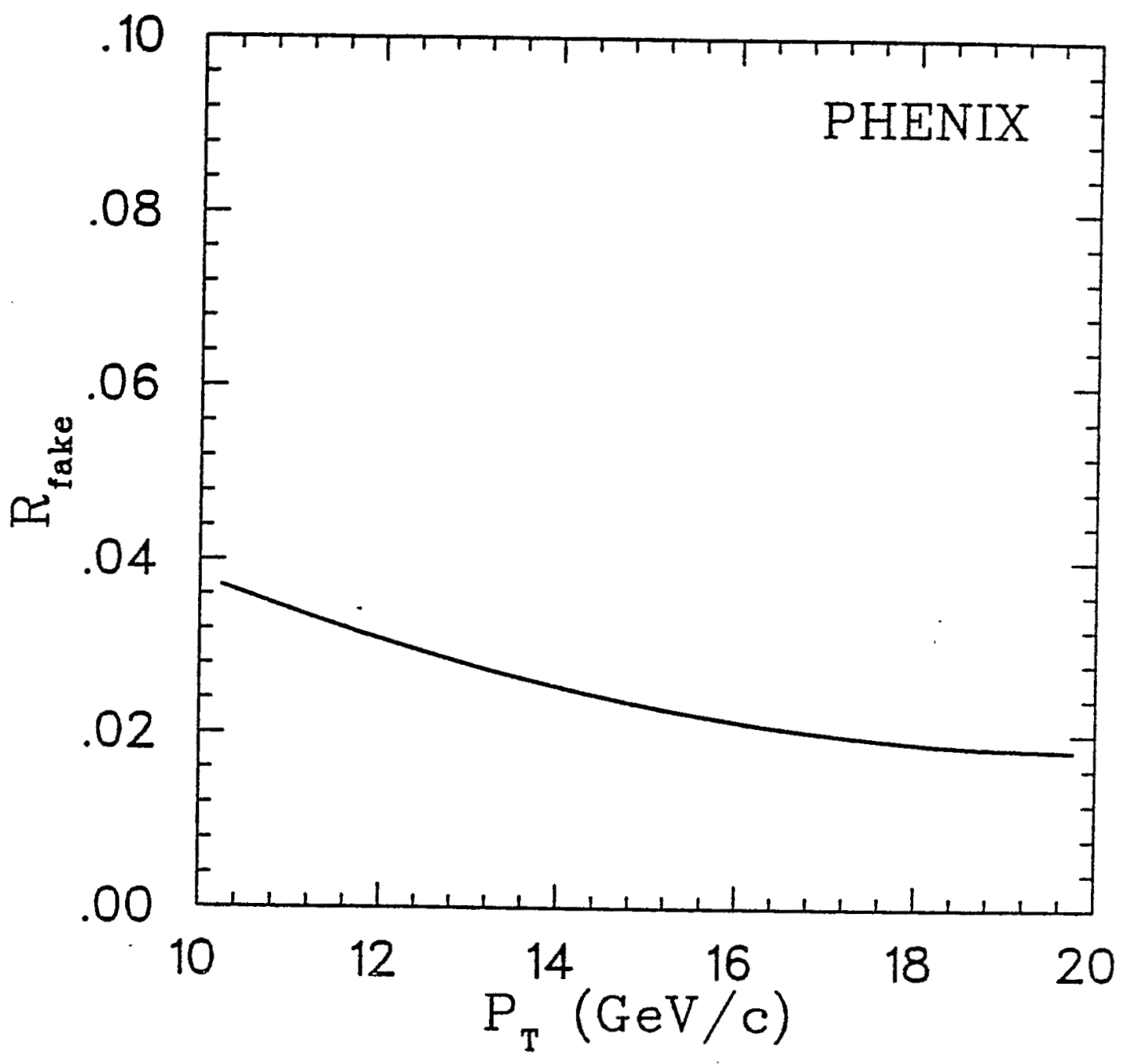

Figure B-9 


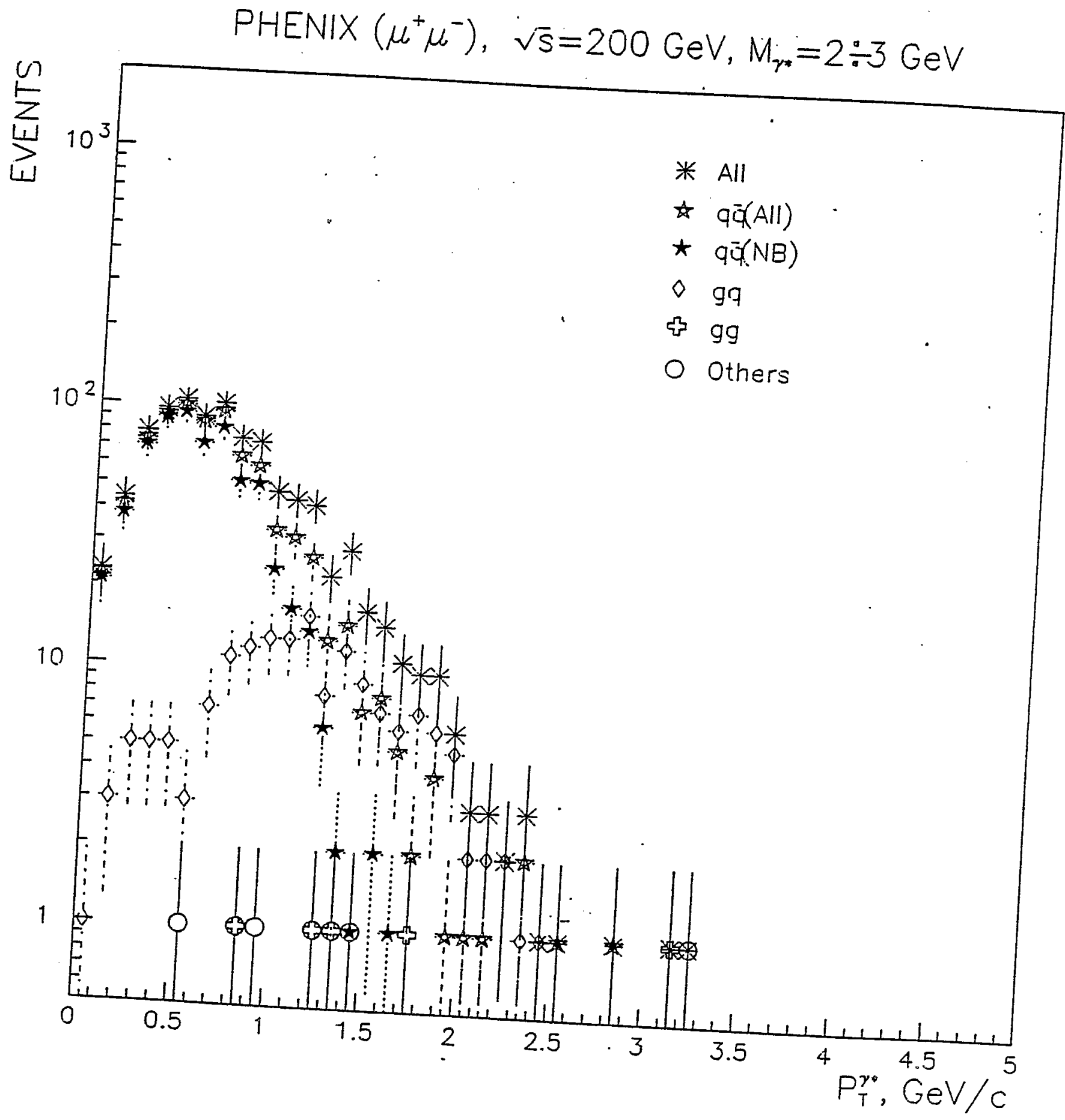



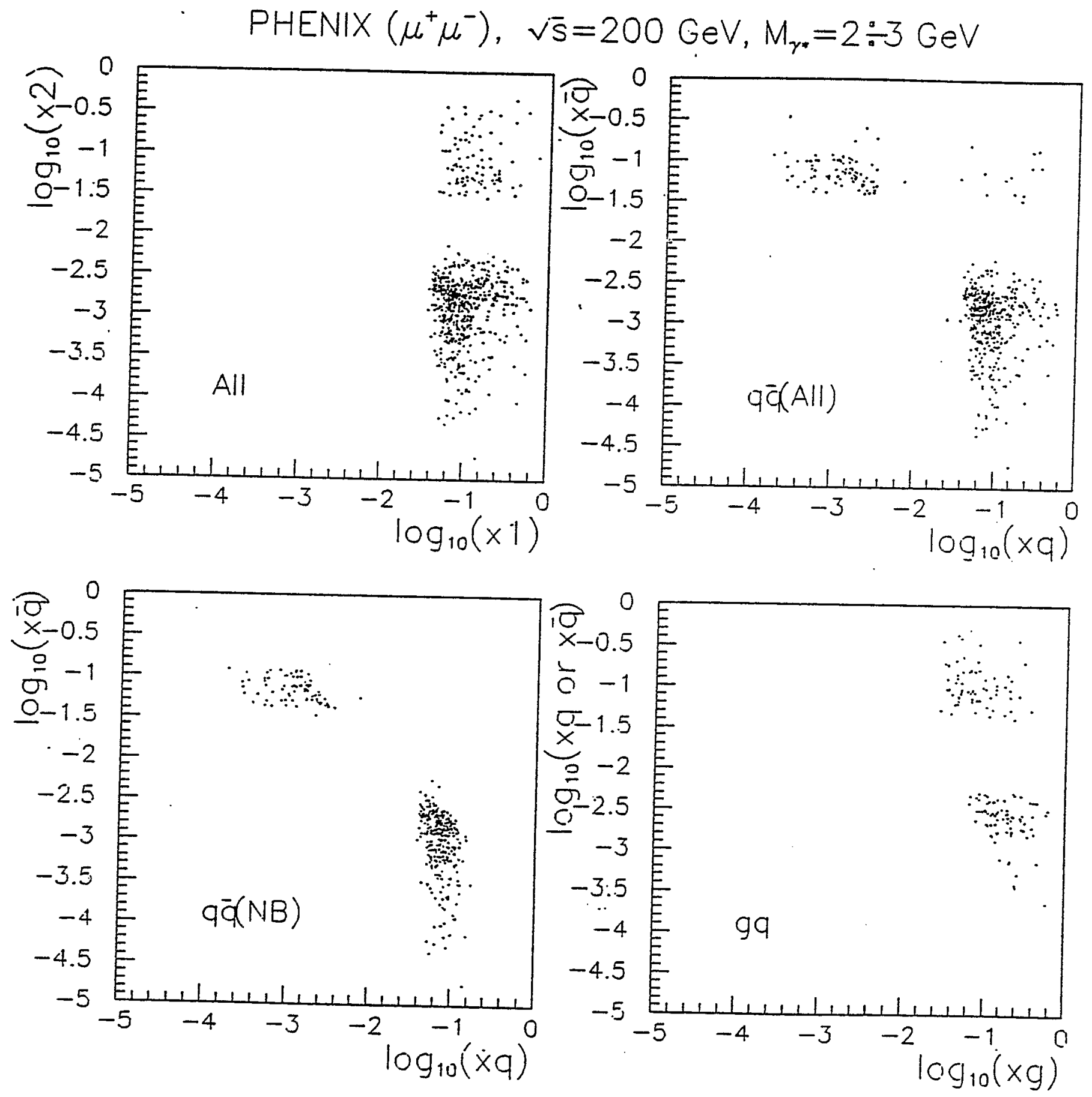

Figure B-11 

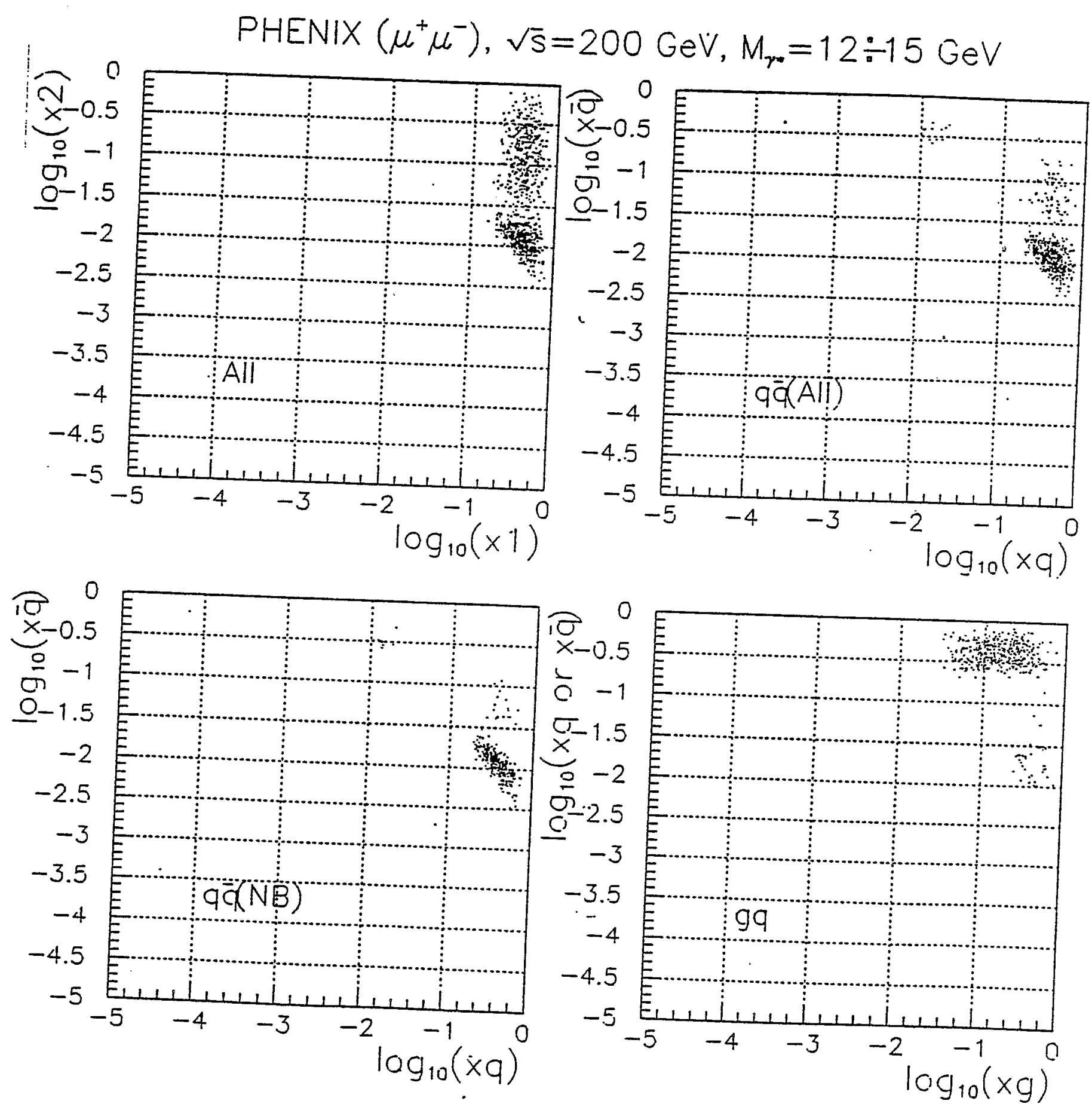

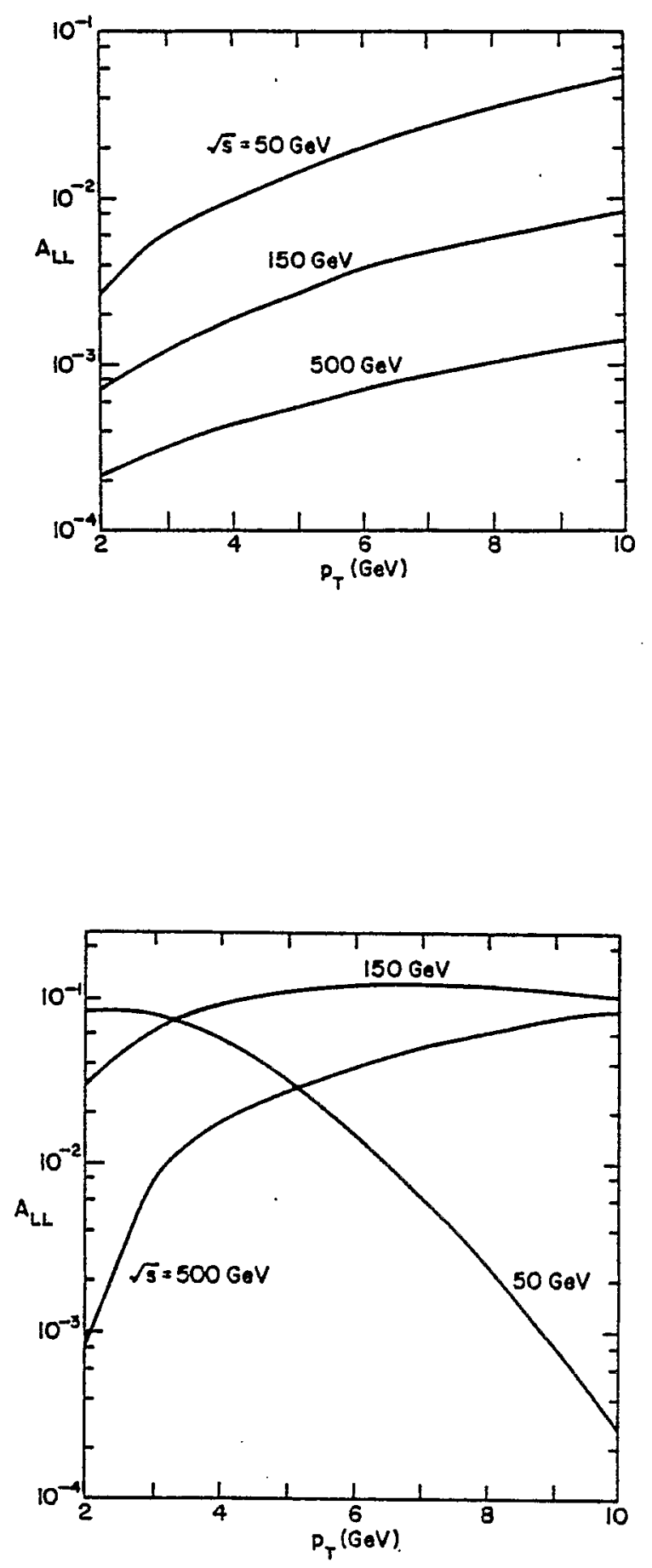

Figure B-13 

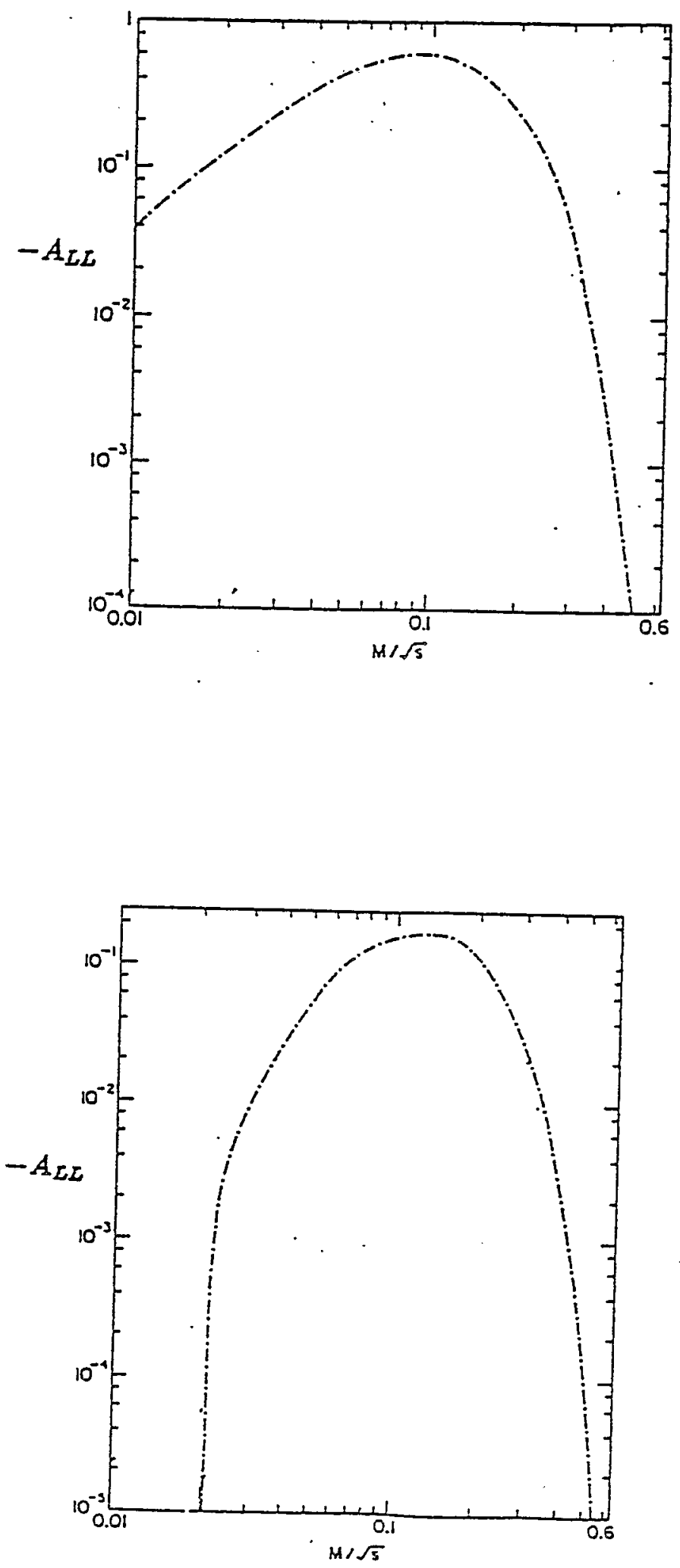

Figure B-14 


\title{
Section C
}

\section{Experiments with the STAR Detector}

\author{
By
}

M. Beddo, D. Grosnick, D. Hill, D. Lopiano, H. Spinka, D. Underwood, A. Yokosawa High Energy Physics Division, Argonne National Laboratory, Argonne, Illinois 60439, USA

P. Buncic, D. Ferenc, K. Kadija, G. Paic, and D. Vranic Rudjer Boskovic Institute, 41001 Zagreb, Croatia

G. Danby, S.E. Eiseman, A Etkin, K.J. Foley, R.W. Hackenburg, M.J. LeVine, R.S. Longacre, W.A. Love, E.D. Platner, P. Rehak, A.C. Saulys, and J.H. Van Dijk Brookhaven National Laboratory, Upton, New York, 11973, USA

H.J. Crawford, J.M. Engelage, and L. Greiner University of California, Berkeley, California 94720, USA

F.P. Brady, J.E. Draper, and J.L. Romero University of California, Davis, California 95616, USA

J.B. Carroll, V. Ghazikhanian, E. Gulmez, T. Hallman, G.J. Igo, S. Trentalange, and C. Whitten, Jr. University of California, Los Angeles, California 90024, USA

M. Kaplan, P.J. Karol, Z. Miolosevich, and E. Vardaci Carnegie Mellon University, Pittsburgh, Pennsylvania 15213, USA

M. Cherney, T.S. McShane, and J. Seger Creighton University, Omaha, Nebraska 68178, USA

M. Gazdzicki, R.E. Renfordt, D. Röhrich, R. Stock, H. Ströbele, and S. Wenig University of Frankfurt, D-6000, Frankfurt am Main 90, Germany

L. Madansky and R. Welsh The Johns Hopkins University, Baltimore, Maryland 21218, USA

B. Anderson, M. L. Justice, D. Keane, Y. Shao, and J. Watson Kent State University, Kent, Ohio 44242, USA

A. S. Akimenko, Yu. I. Arestov, B. V. Chuiko, A. M. Davidenko, A. A. Derevschikov, S. V. Erin, O. A. Grachov, A. K. Likhoded, A. P. Meschanin, S. B. Nurushev, A. I. Ronzhin, V. L. Rykov, A. G. Ufimtzev, A. N. Vasiliev IHEP, Institute for High Energy Physics, Protvino, Russia 
F. Bieser, M.A. Bloomer, D. Cebrta, S.I. Chase, W. Christie, W.R. Edwards, M. Green, D. Greiner, J.W. Harris, H. Huang, P. Jacobs, P. Jones, S. Kleinfelder, P. Lindstrom, S. Margetis, J. Marx, H.S. Matis, C. McParland, J. Mitchell, T. Moore, R. Morse, C. Naudet, T. Noggle, G. Odyniec, D. Olson, A.M. Poskanzer, G. Rai, J. Rasson, H.-G. Ritter, I. Sakrejda, J. Schambach, L.S. Schroeder,

D. Shuman, R. Stone, T.J.M. Symons, H. Wieman, and W.K. Wilson Lawrence Berkeley Laboratory, Berkeley, California 94720, USA

C. S. Chan, M.A. Kramer, S.J. Lindenbaum, K.H. Zhao, and Y. Zhu City College of New York, New York, New York 10031, USA.

A. Aprahamian, N.N. Biswas, U. Garg, V.P. Kenney, and J. Piekarz University of Notre Dame, Notre Dame, Indiana 46556, USA

\section{T. Humanic \\ University of Pittsburgh, Pittsburgh, Pennsylvania 15213, USA}

D.D. Carmony, Y. Choi, A. Hirsch, E. Hjort, N. Porile, R.P. Scharenberg, B. Srivastava, and M. L. Tincknell

Purdue University, West Lafayette, Indiana 47907, USA

D.L. Adams, S. Ahmad, B.E. Bonner, J.A. Buchanan, C.N. Chiou, J.M. Clement, M.D. Corcoran, T. Empl, H.E. Miettinen, G.S. Mutchler, J.B. Roberts, J. Skeens, and I. Stancu Rice University, Houston, Texas 77251, USA

A.D. Chacon, R.K. Choudhury, and K.L. Wolf Texas A\&M University, College Station, Texas 77843, USA

W. Dominik

Warsaw University, Warsaw, Poland

T. Pawlak, W. Peryt, and J. Pluta

Warsaw University of Technology, Warsaw, Poland

R. Bellwied, S. Bennett, J. Bielecki, T.M. Cormier, F. Gang, J. Hall, Q. Li, A. Lukaszew, R. Matheus, C. Pruneau, and G. Roger

Wayne State University, Detroit, Michigan 48201, USA

W. J. Braithwaite, J.G. Cramer, D. Prindle, T.A. Trainor, and X.-Z. Zhu

University of Washington, Seattle, Washington 98195, USA

Z. Fraenkel and I. Tserruya

Weizmann Institute of Science, Rehovot 76100, Israel 


\section{C.1. Phvsics Goals and Capabilities}

Although years of experimental effort at CERN and FNAL colliders have provided much information about the structure of QCD hard scatterings and the parton structure of the proton, there is no corresponding body of data on the spindependence of the elementary interactions and the spin structure of the proton. High-luminosity polarized proton beams in RHIC offer the opportunity to study the unique properties of the spin variable to increase the understanding of these fundamental quantities.

There are several areas of proton-proton spin physics that can be best done with a combination of RHIC and STAR. One of them is the gluon spin structure function with longitudinal spin in the proton. The gluon spin structure function, $\Delta \mathrm{G}$, can be determined crudely with inclusive jets, better with inclusive direct gammas, and best by using a direct gamma in coincidence with the production of an away-side jet and obtaining the $x$ dependence of $\Delta G$. The sea-quark polarization can be determined, as discussed in A.1.1.5, by measuring the longitudinal spin-spin asymmetries in the Drell-Yan process. The quark transversity distributions in a polarized proton have recently been discussed extensively by theorists and it is a fundamental measurement to determine the structure function $h_{1}(x)$ as discussed in A.1.2.1. This can be done by measuring the transverse spin-spin asymmetries in the Drell-Yan process. Single-spin transverse asymmetries in $\pi^{\circ}$ production appear to remain constant at a given $\mathrm{x}_{\mathrm{T}}$ up to $200-\mathrm{GeV} / \mathrm{c}$. At the higher energies available at RHIC, it is unarguable that perturbative QCD should be fully applicable. Finally, the measurement of single-helicity asymmetries (parity violation) in $\mathrm{W}^{ \pm}$ and $\mathrm{Z}^{\circ}$ production as discussed in A.1.3 will be carried out.

We will be able to carry out measurements to accomplish various physics goals discussed in Sections A.1.1, A.1.2, and A.1.3.

i) Several experimental methods to measure direct-gamma and jet production are used in STAR, including fine segmentation and a shower maximum detector in a sampling electromagnetic calorimeter. For these methods, rates and uncertainties on spin-spin observables, such as $A_{L L}$, are estimated for the STAR detector.

In the unpolarized case, inclusive direct-gamma production data have been used to extract information on the gluon structure function $\mathrm{G}(\mathrm{x})$ in the proton. ${ }^{1-6}$ By assuming that the Compton subprocess is the only contribution to the cross section, this subprocess can be expressed in terms of the quark structure func- 
tions in the proton $(u(x), d(x)$, etc., for $u, d, \ldots$ quarks) determined from deep inelastic scattering, 7 the momentum fractions $x_{1}$ and $x_{2}$ (related to the rapidities, $\eta_{\gamma}$ and $\eta_{\text {jet}}$ ), and the parton level cross section calculated from perturbative QCD when the process is a hard scattering. ${ }^{1}$ The onset of hard scattering will be assumed to correspond to $\mathrm{p}_{\mathrm{T}}=10 \mathrm{GeV} / \mathrm{c}$ at $\mathrm{RHIC}$ energies.

The unpolarized gluon structure function has been determined as a function of the momentum fraction $x$ from inclusive direct-gamma + jet experimental data from the ISR. 8 The quark structure function $\mathrm{q}_{\mathrm{i}}(\mathrm{x})$ must be sizable in order to obtain sufficient rate and sensitivity to the gluon structure function. The higher rates will correspond to the $\mathrm{x}$ of the quark in the range $0.02-0.5$, and to the $\mathrm{x}$ of the gluons $<0.4$.

The single inclusive direct-gamma production cross section is an integral over the $(\gamma+$ jet $)$ cross section. In this case it is difficult to extract information on the $\mathrm{x}$-dependence of $\mathrm{G}(\mathrm{x})$. On the other hand, most of the experimental data reported in the literature are for the single inclusive cross section. For the case with spin, similar considerations apply. The analogy of the unpolarized structure functions are the spin-dependent structure functions.

The use of inclusive direct- $\gamma$ and (direct- $\gamma+$ jet) production measurements with longitudinally-polarized protons incident on longitudinally-polarized protons to obtain direct information about $\Delta G(x)$ is described in A.1.1.3 and A.1.1.4. A number of theoretical predictions of spin effects for this process have been made in the past. ${ }^{9-13}$ High-energy inclusive spin predictions are often given for the quantity $A_{L L}$, since such asymmetries are usually measured in spin experiments. Many systematic effects, such as detector efficiencies, absolute luminosity, etc. cancel in $A_{L L}$ experiments. Some of these systematic effects were particularly troublesome for past experiments using solid-state polarized targets that contain substantial fractions of non-hydrogen background nuclei. These effects may be less of a problem with colliding proton beams, since they do not have the background nuclei present. In any case, the uncertainties, $\delta A_{L L}$, for $A_{L L}$ are estimated.

To make first-order estimates of the rates, a number of measurements of the single inclusive direct- $\gamma$ cross sections in $p p$ and $p \bar{p}$ interactions are used. 14-22 Details of estimates of rates and errors for measurements of direct- $\gamma$ and (direct- $\gamma+$ jet) production are described in C.2.1.1, C.2.1.4, C.3.1.1, and also in Ref. 23.

ii) The Drell-Yan process pp $\rightarrow \gamma^{*}+\mathrm{X} \rightarrow \ell+\ell^{-}+\mathrm{X}$ for $\sqrt{\mathrm{s}}=200$ and $500 \mathrm{GeV}$ was studied ${ }^{24}$ by Monte-Carlo simulations with the PYTHIA V5.3 program. ${ }^{25}$ The 
EHLQ126 set of proton structure functions was used. All parameters for PYTHIA were set to their default values except for two. The same values of parameters were used for the study of $\mathrm{pp} \rightarrow \mathrm{Z}^{\circ}+\mathrm{X} \rightarrow \ell^{+} \ell^{-}+\mathrm{X}$ and $\mathrm{pp} \rightarrow \mathrm{W}^{ \pm}+\mathrm{X} \rightarrow \ell^{ \pm} \mathrm{v}+\mathrm{X}$. The results are presented in C.2.3 and C.3.4.

iii) For the following discussion of rates and sensitivities, the integrated luminosity will be taken as

$\mathcal{L}=2 \times 10^{32} \mathrm{~cm}^{-1} \mathrm{sec}^{-2}$ for $4 \times 10^{6}$ seconds (100 days, $50 \%$ efficiency) or

$$
\int \mathcal{L} \mathrm{dt}=8 \times 10^{38} \mathrm{~cm}^{-2} \text { at } 500 \mathrm{GeV} / \mathrm{c}=800 \mathrm{pb}^{-1},
$$

$\mathcal{L}=8 \times 10^{31} \mathrm{~cm}^{-1} \mathrm{sec}$ for $4 \times 10^{6}$ seconds or $\int \mathcal{L} \mathrm{dt}=3.2 \times 10^{38} \mathrm{~cm}^{-2}$ at $200 \mathrm{GeV}$.

The polarization of both beams is taken as $\mathrm{P}=0.7$ and the statistical errors on the asymmetry are given as

$$
\begin{gathered}
\delta A=\frac{1}{\mathrm{P}^{2}} \frac{1}{\sqrt{\mathrm{N}}} \text { for the two-spin asymmetry, and } \\
\delta \mathrm{A}=\frac{1}{\mathrm{P}} \frac{1}{\sqrt{\mathrm{N}}} \text { for the one-spin asymmetry. }
\end{gathered}
$$

\section{C.2. Measurements with Barrel EMC and Shower Maximum Detector}

Both a barrel electromagnetic calorimeter and a shower maximum detector will be required to perform measurements of inclusive direct- $\gamma$, jet, and $\pi^{\circ}$ production. These data will provide information on the helicity and transversity distributions of partons in a polarized proton.

The barrel EM calorimeter (EMC, see Fig. C1) is a lead-scintillator sampling calorimeter. It is located outside the superconducting solenoid coil and inside the iron flux return. It covers $|\eta| \leq 1.05$ and $2 \pi$ in azimuth. At $\eta \sim 0$, the amount of material in front of the EMC is $\sim 1.1 \mathrm{X}_{0}$. The inner radius is 2.53 meters and the length is 6.87 meters. It consists of 60 wedge segments of 6 degrees in $\phi$ and is 
subdivided into 40 pseudo-projective towers over the $\eta$ range. Each tower has 21 layers of lead and scintillator.

The shower maximum detector is similar in design to prototypes that were made for ZEUS, CDF, and SDC. It consists of scintillator strips approximately $1 \mathrm{~cm}$ in $\phi$ by $36 \mathrm{~cm}$ in $\eta$, located at a depth of $\sim 5 \mathrm{X}_{0}$. Each strip is read out by a wavelength-shifting fiber. The longitudinal dimension of the strips corresponds to the physical size of two $\eta$ towers that facilitates bringing the fibers to the outside of the barrel.

\section{Calorimeter Parameters}

\begin{tabular}{|l|l|}
\hline Barrel Calorimeter type & $21 \mathrm{X}_{0}$ lead-scintillator 'EM' section \\
\hline Segmentation: & $\begin{array}{l}60 \text { azimuthal sectors, } \Delta \phi=0.105\left(6^{\circ}\right) \\
40 \text { projective towers in }|\eta|<1.05 \\
\Delta \eta=0.0525\end{array}$ \\
\hline Inner radius: & $2.53 \mathrm{~m}$ \\
\hline Length: & $6.87 \mathrm{~m}$ \\
\hline Weight: & 150 tons \\
\hline Readout: & Waveshifting fiber (2/tile) to PMT \\
\hline PMTs: & 1200 \\
\hline & \\
\hline \hline Shower Max detector: & Scintillator strips parallel to $\eta$ \\
\hline Readout: & Waveshifting fiber \\
\hline
\end{tabular}

\section{C.2.1 Measurements of Parton Helicity Distributions in a Polarized Proton}

Determination of the gluon spin structure function and sea-quark spin structure function is discussed in Section A.1.1.

\section{C.2.1.1 Direct Gamma Production}

As discussed in A.1.1.3, the Compton process ( $q g \rightarrow \gamma q$ ) is expected to dominate inclusive direct- $\gamma$ production by pp interactions. In this case, $A_{L L}$ is directly proportional to $\Delta \mathrm{G}(\mathrm{x})$ as shown in Eq. (A4). Direct photon events, without looking at the away-side jet, provide information about the integral of $\Delta \mathrm{G}(\mathrm{x})$. 
The definition of the spin-spin correlation parameter ALL for inclusive direct- $\gamma$ production is

$$
\mathrm{A}_{\mathrm{LL}}=\frac{1}{\mathrm{P}^{2}} \cdot \frac{\mathrm{N}_{\gamma}^{+}-\mathrm{N}_{\gamma}^{-}}{\mathrm{N}_{\gamma}^{+}+\mathrm{N}_{\gamma}^{-}}
$$

where $\mathrm{P}$ is the beam polarization and $\mathrm{N}_{\gamma}^{ \pm}$are the total number of direct- $\gamma$ events, normalized to the beam luminosity, for beam spins parallel $(+)$ and antiparallel ( - ). Since $\mathbf{N}_{\gamma}^{ \pm}$is not measured directly, it must be estimated from the total counts $\mathbf{n}_{\text {tot }}^{ \pm}$ (total direct $\gamma$ s + total unresolved "fake" $\gamma / s$ ) and from the total number $n_{\pi^{\circ}}^{ \pm}$of reconstructed $\pi^{\circ} s, \eta^{\circ} s$, etc., using

$$
\mathrm{N}_{\gamma}^{ \pm}=\mathrm{n}_{\text {Tot }}^{ \pm}-\mathrm{R}_{\mathrm{fake}} \cdot \mathrm{n}_{\pi^{\circ}}^{ \pm} .
$$

The symbol $R_{\text {fake }}$ (determined by Monte Carlo) in Eq. $C 2$ represents the ratio of the total number of $\gamma$ 's from unresolved $\pi^{\circ}, \eta^{\circ}$, etc. decays to the number of reconstructed $\pi^{\circ}, \eta^{\circ}$, etc. events. Substitution of Eq. C2 into Eq. C1 yields the following relation:

$$
A=A_{L L}=\frac{1}{P^{2}} \cdot \frac{n_{\text {Tot }}^{+}-n_{\text {Tot }}^{-}-R_{\text {fake }}\left(n_{\pi^{*}}^{+}-n_{\pi^{*}}^{-}\right)}{n_{\text {Tot }}^{+}+n_{\text {Tot }}^{-}-R_{\text {fake }}\left(n_{\pi^{*}}^{+}+n_{\pi^{*}}^{-}\right)}
$$

and its error, $\delta A_{L L}$ :

$$
\begin{aligned}
\delta A_{L L}=\frac{1}{\mathrm{P}^{2}} \cdot \frac{1}{\sqrt{\mathrm{N}_{\gamma}}} \cdot & {\left[\left[1-\left(\mathrm{P}^{2} \mathrm{~A}\right)^{2}\right]\right.} \\
& \left.+\mathrm{R}_{\text {fake }} \cdot \frac{\mathrm{N}_{\pi^{\circ}}}{\mathrm{N}_{\gamma}} \cdot\left[1+\left(\mathrm{P}^{2} \mathrm{~A}\right)^{2}-2\left(\mathrm{P}^{2} \mathrm{~A}\right)\left(\mathrm{P}^{2} \mathrm{~B}\right)\right]\right]^{1 / 2} .
\end{aligned}
$$

In the previous expression, $A=A_{L L}$ for inclusive direct- $\gamma$ production and $B$ is the (background) asymmetry in inclusive $\pi^{\circ}, \eta^{\circ}$, etc. production. The quantities $N_{\pi^{\circ}}$ and $N_{\gamma}$ are estimated from Eqs. 20 and 18 in Ref. 23, respectively $\left(N_{\pi^{\circ}} / N_{\gamma} \simeq \sigma \pi^{\circ} / \sigma \gamma\right)$.

The minimum laboratory angle between photon pairs produced through the electromagnetic decay of mesons is given by 


$$
\Delta \theta_{\min }=2 \frac{\mathrm{m}}{\mathrm{E}},
$$

where $m$ is the mass and $E$ is the laboratory energy of the decaying meson. For meson momenta $\mathrm{p}_{\mathrm{m}}$ in the range $10 \leq \mathrm{p}_{\mathrm{m}} \leq 20 \mathrm{GeV} / \mathrm{c}, \Delta \theta_{\min }$ varies from about $14 \mathrm{mr}$ to $27 \mathrm{mr}$ for the $\pi^{\circ}$ decay. Expressed in terms of transverse momentum, $10 \leq \mathrm{p}_{\mathrm{T}} \leq$ $20 \mathrm{GeV} / \mathrm{c}$ and $|\eta| \leq 1$ corresponds to $10 \leq \mathrm{p}_{\mathrm{m}} \leq 31 \mathrm{GeV} / \mathrm{c}$ and to $9 \leq \Delta \theta_{\min } \leq 27 \mathrm{mr}$. Figure C2 shows the distribution of radial energy deposited by $10 \mathrm{GeV}$ photon showers as calculated by the EGS computer program. With very fine segmentation and large distances, the two photons from $\pi^{\circ}$ decay can be distinguished from a single, isolated direct- $\gamma$ event. However, the detector cost increases with finer segmentation or with increasing distance to the photon detector. Furthermore, highly asymmetric decays of mesons may cause the loss of one photon from the detector acceptance or may lead to the inability to identify the low-energy photons because of detector noise. Thus, realistic detectors cannot always distinguish true direct- $\gamma$ events from electromagnetic decays of mesons $\left(R_{\text {fake }} \neq 0\right)$.

The STAR electromagnetic calorimeter design has "towers" with cross sectional area $27 \times 14 \mathrm{~cm}^{2}$ near $\theta_{\mathrm{lab}}=90^{\circ}$, and larger in one dimension at more forward angles. The calorimeter covers $-1.05 \leq \eta \leq 1.05$ and $\Delta \phi=2 \pi$. The inner radius is expected to be about $2.53 \mathrm{~m}$, which gives a lower limit to the angular resolution of two showers of $53 \mathrm{mr}$. This is not sufficient for resolving $\pi^{\circ}$ decays with $10 \leq \mathrm{pT} \leq$ $20 \mathrm{GeV} / \mathrm{c}$ using simple techniques. Since the tower size is so large and the corresponding angular resolution so poor compared to the required values of 9-27 $\mathrm{mr}$, the electromagnetic calorimeter alone will not be useful for direct- $\gamma$ measurements because $R_{\text {fake }}$ would be too large to give meaningful results.

The spatial resolution for showers in the STAR calorimeter could be improved dramatically using a detector made of strips placed at a depth corresponding to the shower maximum in the calorimeter. Simulations were performed using 0.5 , 1 , or 1.5-cm-wide strips of length corresponding to twice the tower dimension in $\eta$ $(28-45 \mathrm{~cm})$ at a radius of $260 \mathrm{~cm}$. In the simulations, it was assumed that the photons could be resolved if $R \Delta \phi \geq 1,2,3 \mathrm{~cm}$, where $R$ is the radius out to the shower maximum and $\Delta \phi$ is azimuthal angle subtended by the photon pair. With the $1-\mathrm{cm}$ strips, the estimated $\delta A_{L L}$ at $\sqrt{\mathrm{s}}=500 \mathrm{GeV}$ is 


$$
\delta A_{L L} \sim \pm \frac{1}{P^{2}}\left(0.0006 \sqrt{8 \times 10^{38} \mathrm{~cm}^{-2} / \int \mathcal{L} \mathrm{dt}}\right) .
$$

At $\sqrt{\mathrm{s}}=200 \mathrm{GeV}$, the uncertainty will be

$$
\delta \mathrm{A}_{\mathrm{LL}} \sim \pm \frac{1}{\mathrm{P}^{2}}\left(0.011 \sqrt{3.2 \times 10^{38} \mathrm{~cm}^{-2} / \int \mathcal{L} \mathrm{dt}}\right) .
$$

The error is increased by a factor of 1.2 for $1.5-\mathrm{cm}$ strips, and is reduced by a factor of 1.3 for $0.5-\mathrm{cm}$ strips. At pT $\sim 20 \mathrm{GeV} / \mathrm{c}$, we note $R \Delta \theta_{\min }=3.5 \mathrm{~cm}$, suggesting that the $1-\mathrm{cm}$ width may be near optimum when cost vs. $\pi^{\circ}$ resolution is considered.

\section{C2.1.2 Single High-p $p_{T}$ Particle Production}

The decays $\pi^{\circ} \rightarrow \gamma \gamma, \eta^{\circ} \rightarrow \gamma$, etc. will be obtained simultaneously with the inclusive direct- $\gamma$ measurements, and in fact are the major background for the direct- $\gamma$ events. The cross section for inclusive $\pi^{\circ}$ production is several times larger, and thus the uncertainties $\delta$ ALL will be somewhat smaller, than for direct- $\gamma$ production. The range of $\mathrm{p}_{\mathrm{T}}$ coverage will also be $10 \lesssim \mathrm{p}_{\mathrm{T}} \lesssim 20 \mathrm{GeV} / \mathrm{c}$.

\section{C2.1.3 Single-Jet Production}

Jets can be detected in STAR with the EM calorimeter for the electromagnetic component, and with the TPC for charged particles. Monte Carlo studies of the rates, acceptance, and resolution were made using the ISAJET program with EHLQ1 structure functions ${ }^{26}$ and GEANT. For these calculations, it was assumed that particles within a cone of radius $\sqrt{(\Delta \eta)^{2}+(\Delta \phi)^{2}}=0.7$ were part of the jet. For the barrel EM calorimeter, the maximum jet rapidity would be $|\eta|<0.3$, corresponding to $0.05<\mathrm{x}<0.3$ at $\mathrm{p}_{\mathrm{T}}=10 \mathrm{GeV} / \mathrm{c}$ and $\sqrt{\mathrm{s}}=200 \mathrm{GeV}$, and to larger $\mathrm{x}$ at higher pT. Sizable rates for jets occur for pT up to $50 \mathrm{GeV} / \mathrm{c}$. The trigger would be the same as for inclusive direct- $\gamma$ events, namely, a cut on the energy detected in a cluster of towers. Figures C3 and C4 show the efficiency of the trigger and the rate as functions of jet $\mathrm{E}_{\mathrm{T}}$ and detector threshold. Figure $\mathrm{C5}$ shows a scatterplot of jet energy from the EM calorimeter plus the TPC versus the jet energy from the EM calorimeter and a hadron calorimeter. The distribution of observed jet energy for jets of $E_{\mathrm{T}}=38-40 \mathrm{GeV}$ (Fig. C6) and of $\mathrm{p}_{\mathrm{T}} \sim 10$ and $15 \mathrm{GeV}$ are given in Fig $\mathrm{C}$.

A simulation of the TPC operation for high-luminosity pp events has begun. Minimum bias events generated by the LUND program ${ }^{25}$ were used to evaluate the effects of pp interactions preceding and following the triggering event. As a result 
of the long TPC readout time ( $50 \mu \mathrm{sec})$, there are thousands of tracks present in a TPC trigger. The number of tracks as a function of radius (TPC readout pad row) are shown as a solid line in Fig. C8 for the generated events at $\sqrt{\mathrm{s}}=500 \mathrm{GeV}$ and both the design $\left(\mathcal{L}=1.4 \times 10^{31} / \mathrm{cm}^{2} / \mathrm{sec}\right)$ and the high luminosities $\left(\mathcal{L}=2 \times 10^{32} /\right.$ $\mathrm{cm}^{2} / \mathrm{sec}$ ). The number of indistinguishable overlapping tracks are indicated by the dotted line. For comparison, a similar calculation with $\mathrm{Au}$ and Au events is shown in Fig. C9. It can be seen that the high-luminosity pp events will not be significantly "worse" than $\mathrm{Au}+\mathrm{Au}$ events. The same conclusion applies to the track momentum resolution shown in Fig. C10 for the two luminosities.

A different way of considering the effects of the background events at high luminosity is shown in Fig. C11. The distribution of the $\mathrm{Z}$ coordinate (along the beam) for the point of closest approach to the beam axis of each reconstructed track is shown. Most tracks correspond to events occurring before or after the triggered event, and thus have their Z-coordinate shifted by the drift time in the STAR TPC. Shaded events in the upper part of Fig. C11 correspond to tracks from the trigger event, which contained two minimum bias events in the same beam crossing. The shaded events in the lower part of the figure correspond to tracks that hit the barrel trigger scintillators outside the TPC. The difference between the shaded tracks in the two parts of Fig. C11 is due to those low-momentum tracks that did not reach the trigger scintillators. It can be seen that there are a considerable number of background tracks in the vicinity of the triggered event. However, the barrel trigger scintillators will provide a powerful constraint to locate the interaction vertex and to identify the proper high-momentum tracks corresponding to the triggering event.

\section{C.2.1.4 Direct-Gamma+ Jet}

The measurement of "gamma + jet" events allows the kinematics of the primary quark-gluon scattering to be determined. The kinematics of the primary partons $\left(\mathrm{x}_{1}\right.$ and $\mathrm{x}_{2}$ ) can be reconstructed by determining the kinematics of the gamma and jet. For the $\mathrm{p}_{\mathrm{T}}$ acceptance of 10 to $20 \mathrm{GeV} / \mathrm{c}, \mathrm{x}_{1}$ and $\mathrm{x}_{2}$ vary from 0.1 to 0.2 at $\sqrt{\mathrm{s}}=200 \mathrm{GeV}$. The cross section for inclusive direct gamma + jet production with $-0.3<\eta_{\text {jet }}<0.3$ and $-1.0<\eta_{\gamma}<1.0$ in the $\mathrm{p}_{\mathrm{T}}$ interval $15-20 \mathrm{GeV} / \mathrm{c}$ is equal to 28 pb.23 For $800 \mathrm{pb}^{-1}$ integrated luminosity, the expected number of detected events is 22,400 , which corresponds to $\delta A_{L L}= \pm 0.013$, ignoring backgrounds. With the barrel calorimeter, the range $-1.0 \leq \eta_{\gamma} \leq 1.0$ can be measured, increasing the number of events and decreasing $\delta A_{L L}$. 
A more complete determination of the gluon spin structure function is described in Section C.3.1.1.

\section{C.2.1.5 Di-Jet Production}

As discussed in A.1.1.2, measurements of $\mathrm{p}^{\uparrow} \mathrm{p}^{\uparrow} \rightarrow \mathrm{Jet}_{1}+\mathrm{Jet}_{2}+\mathrm{X}$ also provide information on $\Delta G$. For low jet-jet invariant masses (MJJ), gluon-gluon subprocesses dominate as shown in Fig. C12. As MJJ increases, gluon-quark and quarkquark scattering become more important. Data on spin-dependent quark and gluon structure functions from deep inelastic lepton scattering and inclusive direct- $\gamma$ production should allow calculation of $A_{L L}$ for inclusive di-jet production as functions of $\mathrm{x}_{1}$ and $\mathrm{x}_{2}$ (defined analogously to the direct- $\gamma$ plus jet case in Section C3.1). Thus these measurements can be considered as consistency checks for the extracted spindependent structure functions or as tests of QCD. The number of di-jet events, Npair, is given in the table below for $\mathrm{pT}_{\mathrm{T}} \geq 10$ and $20 \mathrm{GeV} / \mathrm{c}$ and $|\eta| \leq 0.3$.

\begin{tabular}{|c|c|c|}
\hline \multicolumn{3}{|c|}{ Jet + Jet Events } \\
\hline $\mathrm{pT}_{\mathrm{T}}$ & $\operatorname{|\eta |}$ & $\mathrm{N}$ pair \\
\hline$\geq 10$ & $\leq 0.3$ & $3 \cdot 10^{8}$ \\
\hline$\geq 20$ & $\leq 0.3$ & $7 \cdot 10^{6}$ \\
\hline
\end{tabular}

C.2.2 Measurements of Quark Transversity Distribution in a Polarized Proton

\section{C.2.2.1 $A_{N}$ Measurements in Direct-Gamma and $\pi^{\circ}$ Production}

One-spin transverse asymmetries were previously measured up to $200 \mathrm{GeV} / \mathrm{c}$ using the Fermilab polarized beam. 27 The asymmetry $A_{N}$ in $p^{\uparrow} p \rightarrow \pi^{\circ} X$ with $\mathrm{XF}_{\mathrm{F}}=0$ shows a transition in the production process from a "low-pT" regime with $\mathrm{A}_{\mathrm{N}}=0$ to a "high-p $\mathrm{p}_{\mathrm{T}}$ " regime with $\mathrm{A}_{\mathrm{N}}>0.3$. This transition occurs at $\mathrm{p}_{\mathrm{T}} \approx$ $3.5 \mathrm{GeV} / \mathrm{c}$.

As discussed in A.1.2.2, measurements of $\mathrm{p}^{\uparrow} \mathrm{p} \rightarrow \gamma \mathrm{X}$ and $\mathrm{p}^{\uparrow} \mathrm{p} \rightarrow \pi^{\circ} \mathrm{X}$ will provide important information on the twist-3 parton distribution.

The event rate for direct-gamma production can be estimated in a similar way as C.2.1.1, and then

$$
\delta \mathrm{A}_{\mathrm{N}} \sim \pm \frac{1}{\mathrm{P}}\left(0.014 \sqrt{\left.8 \times 10^{38} \mathrm{~cm}^{-2 / \int \mathcal{L} \mathrm{dt}}\right)} .\right.
$$




\section{C.2.3 Single-Helicity Asymmetry in W and Z Production}

$\mathrm{W}$ and $\mathrm{Z}$ production with polarized protons is a totally unexplored area of experimental high-energy physics. ${ }^{28}$ The predictions for asymmetries, in particular for the parity nonconservation ones, are expected to be larger than twenty percent, 2930 and these predictions are dependent on our understanding of the proton structure. Thus this measurement studies the QCD structure of the proton as well as confirming the Standard Model using polarized-hadron interactions.

The estimated PYTHIA V5.3 production cross sections for $\mathrm{W}$ and $\mathrm{Z}$ in pp interactions at $\sqrt{\mathrm{s}}=500 \mathrm{GeV}$ are as follows (see Section C.3.2 and Ref. 24):

$$
\begin{aligned}
& \sigma \mathrm{B}\left(\mathrm{pp} \rightarrow \mathrm{W}^{+}+\mathrm{X} \rightarrow \mathrm{e}^{+}+\mathrm{v}+\mathrm{X}\right)=120 \mathrm{pb} \\
& \sigma \mathrm{B}\left(\mathrm{pp} \rightarrow \mathrm{W}^{-}+\mathrm{X} \rightarrow \mathrm{e}^{-}+\bar{v}+\mathrm{X}\right)=43 \mathrm{pb} \\
& \sigma \mathrm{B}\left(\mathrm{pp} \rightarrow \mathrm{Z}^{\circ}+\mathrm{X} \rightarrow \mathrm{e}^{+} \mathrm{e}^{-}+\mathrm{X}\right)=10 \mathrm{pb}
\end{aligned}
$$

The STAR detector with electromagnetic calorimeters is especially suitable for experiments with the $\mathrm{W}$ and $\mathrm{Z}$ due to its large acceptance for electrons produced by high mass particle decays. The estimated STAR barrel calorimeter acceptance is about $66 \%$ for $\mathrm{W}$, and $47 \%$ for $\mathrm{Z}$. With the additional endcap calorimeters these values increase to $92 \%$ and $88 \%$ respectively.

In the table below, the event rate estimates at $\sqrt{\mathrm{s}}=500 \mathrm{GeV}$ for the integrated luminosity $800 \mathrm{pb}^{-1}$ are presented.

\begin{tabular}{|c|c|}
\hline Boson & STAR (Barrel) \\
\hline $\mathrm{W}^{+}+\mathrm{W}^{-}$ & 83,000 \\
$\mathrm{~W}_{+}$ & 61,000 \\
$\mathrm{~W}_{-}$ & 22,000 \\
\hline $\mathrm{Z}^{\circ}$ & 3,840 \\
\hline
\end{tabular}

\section{C.3. Measurements with Endcaps}

An important upgrade for the photon detection would be a pair of EM endcap calorimeters to be placed inside the iron pole piece as shown in Fig. C1. They would cover $|\eta| \leq 2.0$. 


\section{C.3.1 Measurements of $\Delta G(x)$, the Gluon Spin Structure Function}

\section{C.3.1.1 Detecting the Direct- $\gamma$ and the "Away-Side" Jet}

In order to measure $\mathrm{G}(\mathrm{x})$, both the direct- $\gamma$ and the "away-side" jet must be detected in coincidence so that the kinematics of the incoming partons can be calculated. Rates are estimated by requiring acceptance about the jet direction corresponding to be $\sqrt{(\Delta \eta)^{2}+(\Delta \phi)^{2}}=0.7$. Thus, a central barrel calorimeter extending to $|\eta| \leq 1.0$ will detect jets only for $\left|\eta_{\text {Jet }}\right| \leq 0.3$, and a combined barrel and endcap calorimeter with $|\eta| \leq 2.0$ would allow jet detection to $\left|\eta_{\mathrm{Jet}}\right| \leq 1.3$.

The Compton and annihilation subprocesses both involve $2 \rightarrow 2$ scatterings. The incoming partons are assumed to have fractions $x_{1}$ and $x_{2}$ of the beam momentum and collide colinearly. Then $x_{1}$ and $x_{2}$ are given in terms of pseudorapidity as:

$$
\begin{aligned}
& \mathrm{x}_{1} \simeq \frac{2 \mathrm{P}_{\mathrm{T}}}{\sqrt{\mathrm{s}}}\left(\frac{\mathrm{e}^{\eta_{1}}+\mathrm{e}^{\eta_{2}}}{2}\right), \\
& \mathrm{x}_{2} \simeq \frac{2 \mathrm{P}_{\mathrm{T}}}{\sqrt{\mathrm{s}}}\left(\frac{\mathrm{e}^{-\eta_{1}}+\mathrm{e}^{-\eta_{2}}}{2}\right) .
\end{aligned}
$$

Figure C13 shows the $\mathrm{x}$ coverage for $\mathrm{x}_{\mathrm{T}}=2 \mathrm{P}_{\mathrm{T}} / \sqrt{\mathrm{s}}=0.1$ as functions of the direct- $\gamma$ and jet pseudorapidities.

Assuming the dominance of the Compton subprocess ( $q g \rightarrow \gamma q$ ), one $x_{i}$ must be larger than 0.2 to 0.3 so that the incoming quark has a sizable polarization. The other $x_{i}$ would then correspond to the momentum fraction of the incoming gluon. For events with $10 \leq \mathrm{p}_{\mathrm{T}} \leq 20 \mathrm{GeV} / \mathrm{c}$ and $\mathrm{x}_{\mathrm{i}}>0.2$ and no endcaps, the range of $\mathrm{x}_{2}$ is very limited except at low $\sqrt{\mathrm{s}}$, where the cross section and luminosity are small. With an endcap, there is reasonable $\mathrm{x}_{2}$ range available below $\sqrt{\mathrm{s}} \sim 300 \mathrm{GeV}$ for $\mathrm{p}_{\mathrm{T}}=$ $10 \mathrm{GeV} / \mathrm{c}$, and a very broad $\mathrm{x}_{2}$ range for $\mathrm{pT}_{\mathrm{T}}=20 \mathrm{GeV} / \mathrm{c}$.

The expected event rate in STAR was calculated using the ISAJET program with EHLQ1 structure functions ${ }^{26}$ and GEANT. Estimates of $\delta A_{L L}$ for various $x$ regions are made at $\sqrt{\mathrm{s}}=200 \mathrm{GeV}$ as shown in the table below. The rows show various $\min \left(\mathrm{x}_{1}, \mathrm{x}_{2}\right)$ bins (roughly corresponding to the gluon) and the columns show various $\max \left(x_{1}, x_{2}\right)$ bins (roughly corresponding to the quark). In the calculation, an ideal detector with endcaps and acceptances of $\left|\eta_{\gamma}\right| \leq 2,\left|\eta_{\text {jet }}\right| \leq 1.3, \mathrm{p}_{\mathrm{T}} \geq 10$ 
$\mathrm{GeV} / \mathrm{c}$ were assumed. The errors listed have been scaled for 1-RHIC-month and normalized with a beam polarization of $P=0.7$. The ISAJET total cross section was $1894 \mathrm{pb}$.

Error Asymmetry, $\delta A_{L L}$, for Incoming Partons with $x_{1}$ and $x_{2}$ at $\sqrt{s}=200 \mathrm{GeV}$

\begin{tabular}{|c|c|c|c|c|}
\hline & \multicolumn{4}{|c|}{$\max \left(x_{1}, x_{2}\right)$} \\
\hline $\min \left(x_{1}, x_{2}\right)$ & $\leq 0.2$ & $0.2-0.3$ & $0.3-0.4$ & $>0.4$ \\
\hline $0.00-0.05$ & & 0.012 & 0.009 & 0.007 \\
\hline $0.05-0.10$ & 0.006 & 0.006 & 0.010 & 0.012 \\
\hline $0.10-0.15$ & 0.007 & 0.010 & 0.020 & 0.030 \\
\hline $0.15-0.20$ & 0.021 & 0.019 & 0.038 & 0.060 \\
\hline$>0.20$ & & 0.035 & 0.049 & 0.073 \\
\hline
\end{tabular}

\section{C.3.1.2 Di-jet Production}

This subject has already been discussed in A.1.1.2 and C.2.1.5. Rates for inclusive di-jet production are hundreds of times larger than direct- $\gamma$ plus jet production. The endcap calorimeter is essential to obtain a sizable range of pseudorapidity for the jets. With $\left|\eta_{1}\right|,\left|\eta_{2}\right| \leq 1.3$ and $\mathrm{p}_{\mathrm{T} \text { jet }}>10 \mathrm{GeV} / \mathrm{c}$, the cross section at $\sqrt{\mathrm{s}}=200 \mathrm{GeV}$ was estimated from the ISAJET program to be $\sigma=5200 \mathrm{nb}$.

\begin{tabular}{|c|c|c|}
\hline \multicolumn{3}{|c|}{ Jet + Jet Events } \\
\hline $\mathrm{p}_{\mathrm{T}}$ & $|\eta|$ & N pair \\
\hline$\geq 10$ & $\leq 1.3$ & $4 \cdot 10^{9}$ \\
\hline$\geq 20$ & $\leq 1.3$ & $2 \cdot 10^{8}$ \\
\hline
\end{tabular}

\section{C.3.2 Measurements of the Parton Helicity Distribution in the Drell-Yan Process}

The purpose of these measurements is to study the sea-quark polarization as discussed in Section A.1.1.5. 
We find the $q \bar{q}$ dominant region of $p p \rightarrow\left(e^{+} e^{-}\right) x$ for high masses produced at PT $<1 \mathrm{GeV} / \mathrm{c}$ with the formula for $A_{L L}$ given in Eq. (A5). Larger values of $A_{L L}$ are expected at higher $\mathrm{xF}$. The $\mathrm{x}_{\mathrm{F}}$ acceptance is about $0.17-0.20$, where $\mathrm{x}_{1}$ and $\mathrm{x}_{2}$ have reasonable quark and sea-quark polarizations. Here, one expects an asymmetry of about $A_{L L}=+15 \%$, assuming a negative sea-quark polarization.

The kinematics of the events detected along with the contributions of the main subprocesses are shown in Figs. C14 and C15.

From the EMC data, we learn that the net spin carried by gluons and the sea of $q \bar{q}$ pairs is constrained by the relation

$$
\Delta s-\left(\alpha_{\mathrm{g}} / 4 \pi\right) \Delta \mathrm{G}=-0.11 \pm 0.03 \text {. }
$$

This suggests that gluons and/or sea quarks may be strongly polarized in a polarized proton. To test various proposed schemes for $\Delta s$, the Drell-Yan experiments with polarized proton beams are suitable.

This issue has been discussed in Ref. 31 , and a sizable asymmetry was predicted for a kinematics region of $\sqrt{\tau}=\mathrm{M}_{\mathrm{ee}} / \sqrt{\mathrm{s}}$ more than 0.3 , which is not easily accessible. The mass region accessible with STAR detector is of $\tau \approx 0.1$, and the expected asymmetry, $A_{L L} \approx 5 \%$. What is essential, however, is that the sign of this asymmetry is opposite in the cases of a polarized and unpolarized sea. Even a measurement of the sign of $A_{L L}$ will be very valuable, since it will indicate whether the sea quarks of the proton are unpolarized or polarized negatively. This question can be answered by the measurements of $A_{L L}$ with accuracy about 1-1.5\%, which, as shown below, is reachable in STAR.

The Drell-Yan pair production had been studied by Monte-Carlo simulations using PYTHIA V5.3 of the LUND set of routines. ${ }^{25}$ The EHLQ1 set of parton structure functions ${ }^{26}$ have also been used. The event rate estimates for $\mathrm{e}^{+} \mathrm{e}^{-}$DrellYan pairs in STAR at $\sqrt{\mathrm{s}}=200 \mathrm{GeV}$ with and without the endcap calorimeter are presented in the table below for the integrated luminosity of $800 \mathrm{pb}^{-1}$ and the triggering cut of electron $\mathrm{pT}_{\mathrm{T}}>4 \mathrm{GeV} / \mathrm{c}^{24}$

\begin{tabular}{|l|c|c|c|c|c|c|}
\hline $\mathrm{M}_{\mathrm{ee}}, \mathrm{GeV} / \mathrm{c}^{2}$ & $5-9$ & $9-12$ & $12-15$ & $15-20$ & $20-25$ & $5-25$ \\
\hline Without Endcaps & 33,000 & 21,000 & 8,500 & 5,500 & 1,900 & 70,000 \\
\hline With Endcaps & 71,000 & 50,000 & 21,000 & 13,500 & 4,300 & 160,000 \\
\hline
\end{tabular}


As an example, in the mass region $15-25 \mathrm{GeV} / \mathrm{c}^{2}, \sqrt{\tau}=0.075-0.125$, and the expected numbers of $e^{+} e^{-}$pairs are 7,400 and 17,800 for STAR without and with the endcap calorimeter respectively. This corresponds to the reachable levels for $\delta A_{L L}$ of $2.3 \%$ and $1.5 \%$. One can see that the STAR detector is quite suitable using the entire mass region for the polarization measurements of the Drell-Yan process, and the endcap calorimeters considerably improve the accuracy of the measurements.

The theoretical predictions for $A_{L L}$ mentioned above certainly are model dependent. The experience from previous polarization experiments shows that these measurements can quite often produce very unexpected results. The accuracy of 1.5 $-2.0 \%$ is certainly rather high for high-energy physics polarization experiments in general.

\section{C.3.3 Measurements of $h_{1}(x)$ in the Drell-Yan Process}

There are also interesting predictions for the Drell-Yan pairs produced by transversely-polarized protons. The importance of determining $h_{1}(x)$ was discussed in Section A.1.2.1. The transverse double-spin asymmetry is related to $h_{1}(x)$ as shown in Eq. (A7). At $\sqrt{\mathrm{s}}=200 \mathrm{GeV}$, we expect the number of useful events for $A_{N N}$ to be 70,000 at mass region of 5 to $9 \mathrm{GeV}$.

\section{C.3.4 W and ZProduction}

The physics justification for the study of $\mathrm{W}^{+}$and $\mathrm{W}^{-}$is described in Section A.1.3.

The acceptance for $\mathrm{W}^{ \pm}$production is close to $95 \%$. In the table below, the event-rate estimates at $\sqrt{\mathrm{s}}=500 \mathrm{GeV}$ are presented.

\begin{tabular}{|c|c|}
\hline Boson & Barrel and Endcaps \\
\hline $\mathrm{W}^{+}+\mathrm{W}^{-}$ & 110,000 \\
$\mathrm{~W}_{+}$ & 80,000 \\
$\mathrm{~W}_{-}$ & 30,000 \\
\hline $\mathrm{Z}^{\circ}$ & 7,200 \\
\hline
\end{tabular}




\section{C.4 Cost Estimates for STAR Upgrades for Spin Physics}

The following estimates are taken from the STAR Conceptual Design Report and the STAR WBS. Included are costs for the barrel EM calorimeter with shower maximum detector, front-end electronics, data acquisition and computing, and installation and testing.

Materials (K\$)

Manufacturing Labor (K\$)

EDIA (Engineering, Design, etc.) (K\$)

Contingency $(28.6 \%)(\mathrm{K} \$)$

Grand Total Cost in (K\$)
4720

1160

1183

$\underline{2017}$

9080

Note: Some of the assembly, engineering, and design could be done by members of the STAR collaboration, which will reduce this cost.

An additional important upgrade for spin physics would be a pair of endcap EM calorimeters, Detailed designs and costs are not available. A very crude estimate of the cost is $\sim \$ 2200 \mathrm{~K}$ per end cap.

\section{C.5 Schedules and Requests}

It is desired to build the EM calorimeter on a time scale compatible with the rest of the STAR Phase 1 detector. This would require substantial funding for engineering and prototypes in FY1993 and for the lead, scintillator, fiber support structure and fixtures, as well as labor, for FY1994 and FY1995. Much of the funding for electronics and data acquisition could be deferred until FY1995 - 1997, after the calorimeter modules were physically installed in STAR. 


\section{References}

\section{Section C}

1) J. F. Owens, Rev. Mod. Phys. 59, 465 (1987).

2) L. Cormell and J. F. Owens, Phys. Rev. D22, 1609 (1980).

3) F. Halzen, M. Dechantsreiter, and D. M. Scott, Phys. Rev. D22, 1617 (1980).

4) R. Baier, J. Engels, and B. Peterson, Z. Phys. C6, 309 (1980).

5) A. P. Cantogouris, S. Papadopoulos, and C. Papavassiliou, Nucl. Phys. B179, 461 (1981).

6) R. R. Horgan and P. N. Scharbach, Nucl. Phys. B181, 421 (1981).

7) For example, see J. G. Morfin and W. K. Tung, Z. Phys. C52, 13 (1991).

8) T. Akesson et al., Z. Phys. C34, 293 (1987). (ISR - AFS Collaboration)

9) N. S. Craigie, K. Hidaka, M. Jacob, and F. M. Renard, Phys. Rep. C99, 69 (1983).

10) M. B. Einhorn and J. Soffer, Nucl. Phys. B274, 714 (1986).

11) E. L. Berger and J. Qiu, Phys. Rev. D40, 778 (1989); 3128 (1989); in Proc. of the Polarized Collider Workshop, Penn State University, 1990, edited by J. Collins, S. Heppelman, and R. Robinett (AIP Conf. Proc. No. 223, American Institute of Physics, New York, 1991), p. 162.

12) P. Matthews and R. Ramachandran, Z. Phys. C53, 305 (1992).

13) M. Gluck and W. Vogelsang, Phys. Lett. B277, 515 (1992).

14) C. De Masrzo et al., Phys. Rev. D36, 8 (1987). (NA24 Collaboration).

15) M. Bonesini et al., Z. Phys. C38, 371 (1988). (WA70 Collaboration).

16) J. L. Cortes, B. N. Pire, and J. Ralston, Ecole Polytechnique Preprint A048.0491 (1991).

17) A. Bernasconi et al., Phys. Lett. B206, 163 (1988). (UA6 Collaboration). 
18) A. L. S. Angelis, et al., Phys. Lett. B94, 106 (1980). (CCOR - R110 Collaboration).

19) E. Anassontzis et al., Z. Phys. C13, 277 (1982). (ISR - R806 Collaboration)

20) R. Ansari et al., Phys. Lett. B176, 239 (1986). (UA2 Collaboration).

21) C. Albajar et al., Phys. Lett. B209, 385 (1988). (UA1 Collaboration).

22) F. Abe et al., Phys. Rev. Lett. 68, 2734 (1992). (CDF Collaboration).

23) M. E. Beddo, H. Spinka, and D. G. Underwood, ANL-HEP-TR-92-59, STAR Note \# 77.

24) A. A. Derevschikov and V. L. Rykov, Internal RSC Report RSC-BNL/IHEP-4, Brookhaven, August 1992,

25) H. U. Bengtsson and T. Sjostrand, A Manual to PYTHIA V5.3 in "The LUND Monte Carlo Programs Long Writeup," Pool programs W5035/W5046/W5047/ W5048, CERN, 1 November 1989.

26) E. Eichten, I. Hinchliffe, K. Lane, and C. Quigg, Rev. Mod. Phys. 56, 579 (1984); 58, 1065 (E) (1986).

27) D. L. Adams et al., Phys. Lett. B276, 531 (1992).

28) M. J. Tannenbaum, in Proc. of the Polarized Collider Workshop, Penn State University, 1990, edited by J. Collins, S. Heppelmann, and R. Robinett (AIP Conf. Proc. No. 223, American Institute of Physics, New York, 1991), p. 201.

29) C. Bourrely, J. Ph. Guillet, and J. Soffer, Nucl. Phys. B361, 72 (1991).

30) E. Richter-Was, Phys. Rev. D34, 2893 (1986).

31) Hai-Yang Cheng and Sheng-Nan Lai., Phys. Rev. D41, 91 (1990). 


\section{Figure Captions}

\section{Section C}

Figure 1 Barrel calorimeter and endcap calorimeters (ECEM UPGRADE) in the STAR detector.

Figure 2 Energy deposited into polystyrene as a function of radial distance for $10 \mathrm{GeV}$ photon showers using EGS.

Figure 3 The trigger efficiency as a function of jet energy for events containing a jet with a given single tower discrimination threshold. Data shown are for pp collisions at $\sqrt{\mathrm{s}}=200 \mathrm{GeV} / \mathrm{c}$.

Figure 4 The rate per second per $\mathrm{GeV}$ of events containing jets of a given $\mathrm{E}_{t}$ for various single tower discrimination thresholds. Data shown are for $\mathrm{pp}$ collisions at $\sqrt{\mathrm{s}}=200 \mathrm{GeV}$.

Figure 5 A scatterplot of the energy of clusters found using electromagnetic energy from the STAR EMC plus tracking and momentum information from the TPC versus EM plus hadronic calorimetry. Data shown are for $40 \mathrm{GeV}$ jet events from pp collisions at $\sqrt{\mathrm{s}}=200 \mathrm{GeV}$. The asymmetry in the distribution is due primarily to the transverse energy of neutrons and $\mathrm{K}_{\mathrm{L}}^{\circ}$ mesons that are detected using EM plus hadronic calorimetry, but are detected in the EMC alone with low probability.

Figure 6 A projection on the EM plus tracking axis of a slice of the EM plus hadronic calorimetry axis for jets with $\mathrm{E}_{\mathrm{T}}$ from 38 to $40 \mathrm{GeV}$.

Figure 7 Distribution of observed jet energy for jet $\mathrm{pT}=10$ and $15 \mathrm{GeV} / \mathrm{c}$.

Figure 8 TPC hit density ( $p+p$ collisions) as a function of radius (pad row). 
Figure 9 Total hit multiplicity as a function of (pad row) for a central $\mathrm{Au}+\mathrm{Au}$ event.

Figure 10 Track momentum resolution ( $p+p$ collisions) for the design and high luminosities.

Figure 11 Histogram of the $\mathrm{Z}$ coordinate of reconstructed tracks in the TPC for $\mathcal{L}=2 \times 10^{32} \mathrm{~cm}^{-2} \mathrm{sec}^{-1}$. The shaded area in the upper part of the figure are tracks from the triggered event, and in the lower part are tracks that reach the barrel trigger scintillators outside the TPC.

Figure 12 Contribution of the different subprocesses versus the invariant di-jet mass at $\sqrt{\mathrm{s}}=200 \mathrm{GeV}$.

Figure 13 Plots of $x_{1}$ and $x_{2}$ as functions of the direct- $\gamma$ and jet pseudorapidities. The solid lines represent contours for $\mathrm{x}_{1}$ and the dashed lines are contours for $\mathbf{x}_{2}$.

Figure 14 The pT $\left(\mathrm{e}^{+} \mathrm{e}^{-}\right)$distribution of the Drell-Yan pairs detected in STAR with the endcap calorimeter. The contributions of the main subprocesses are shown. The $q \bar{q}(\mathrm{NB})$ notion marks the contribution of the nonbremsstrahlunged $\mathrm{q} \overline{\mathrm{q}}$ annihilation of primary partons.

Figure 15 The $\mathrm{x}_{\mathrm{bj}}$ distributions of the primary partons producing Drell-Yan $\mathrm{e}^{+} \mathrm{e}^{-}$ pairs detected in STAR with endcap calorimeter. 


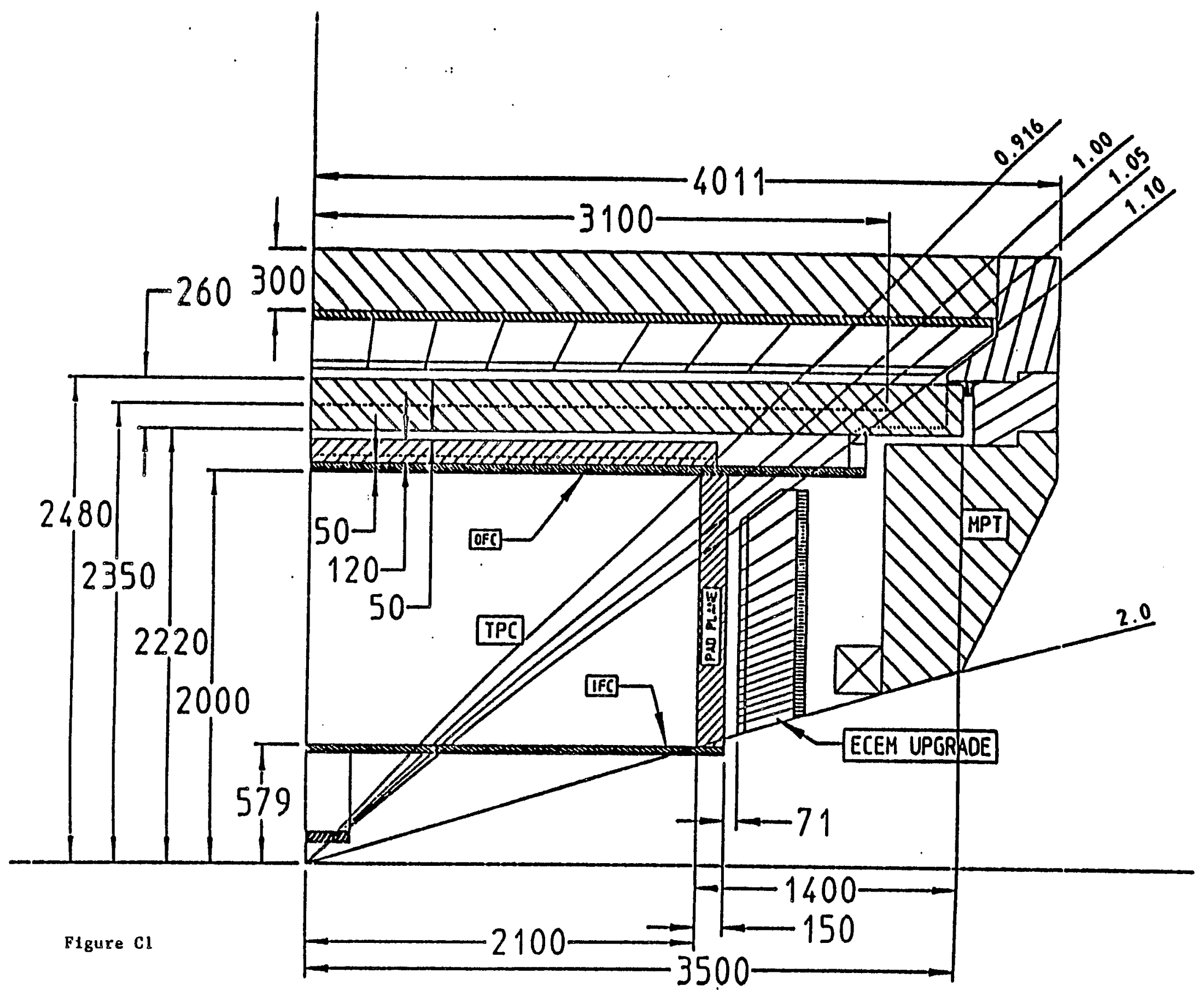




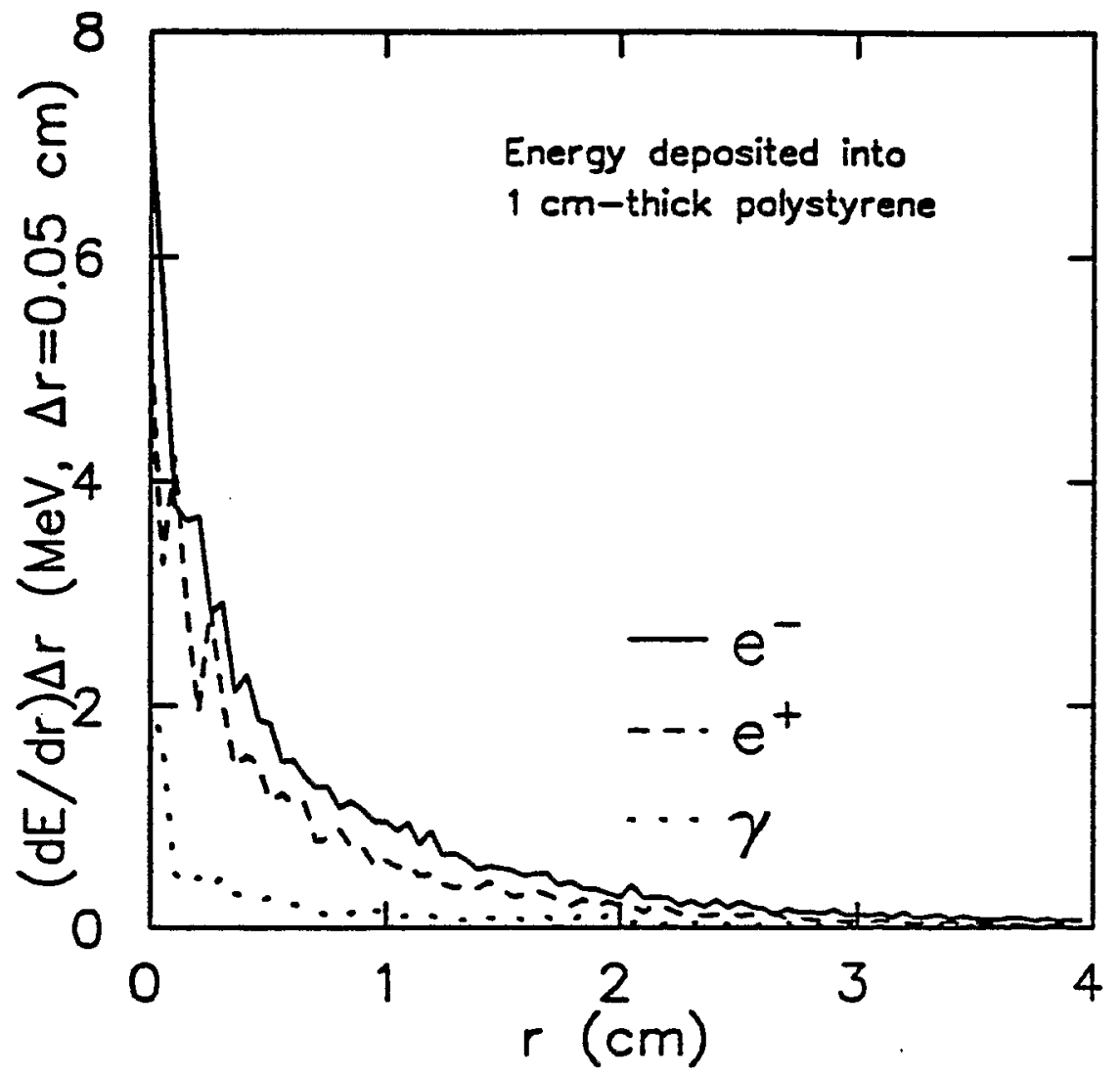

Figure C2

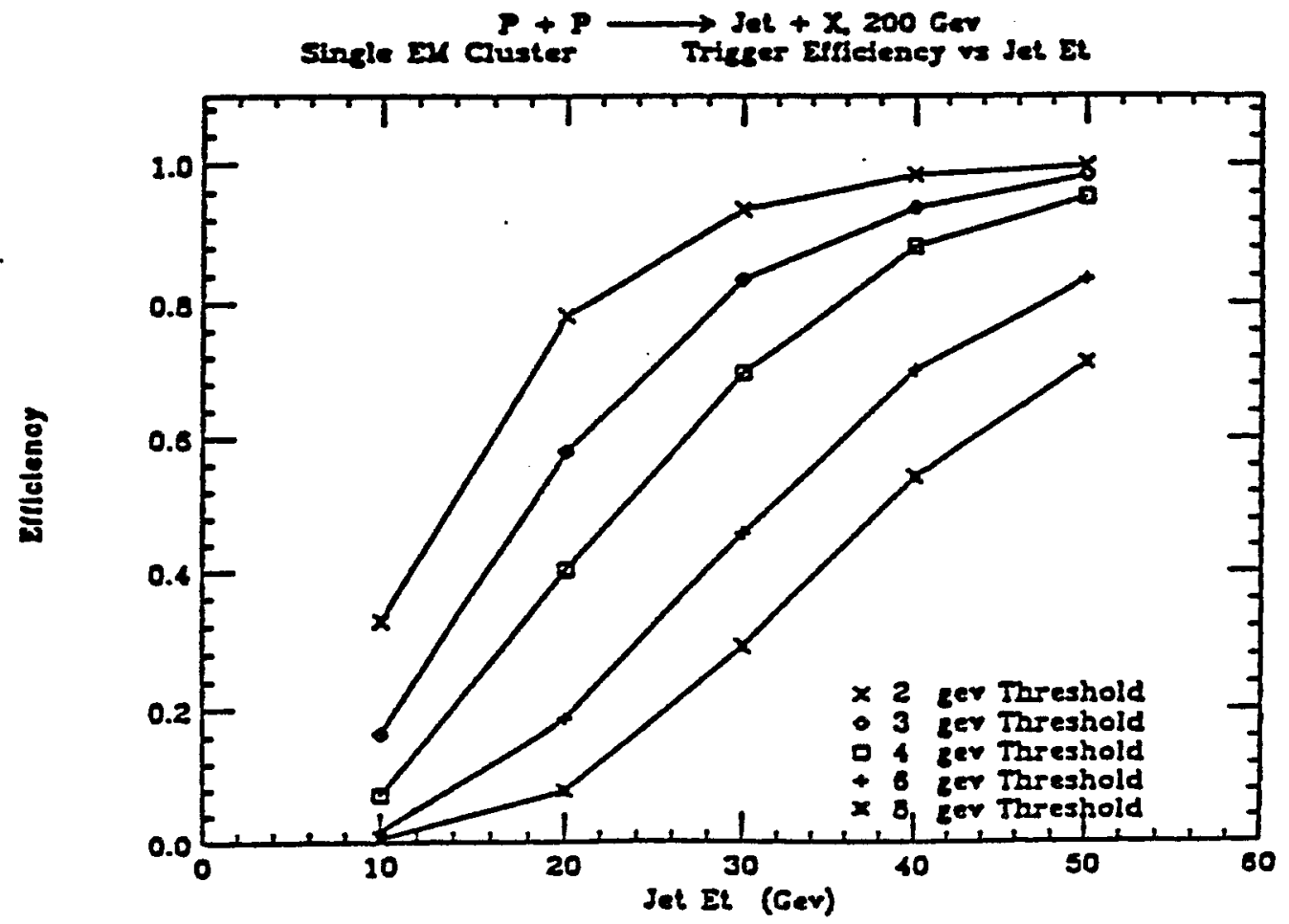

Figure C3 


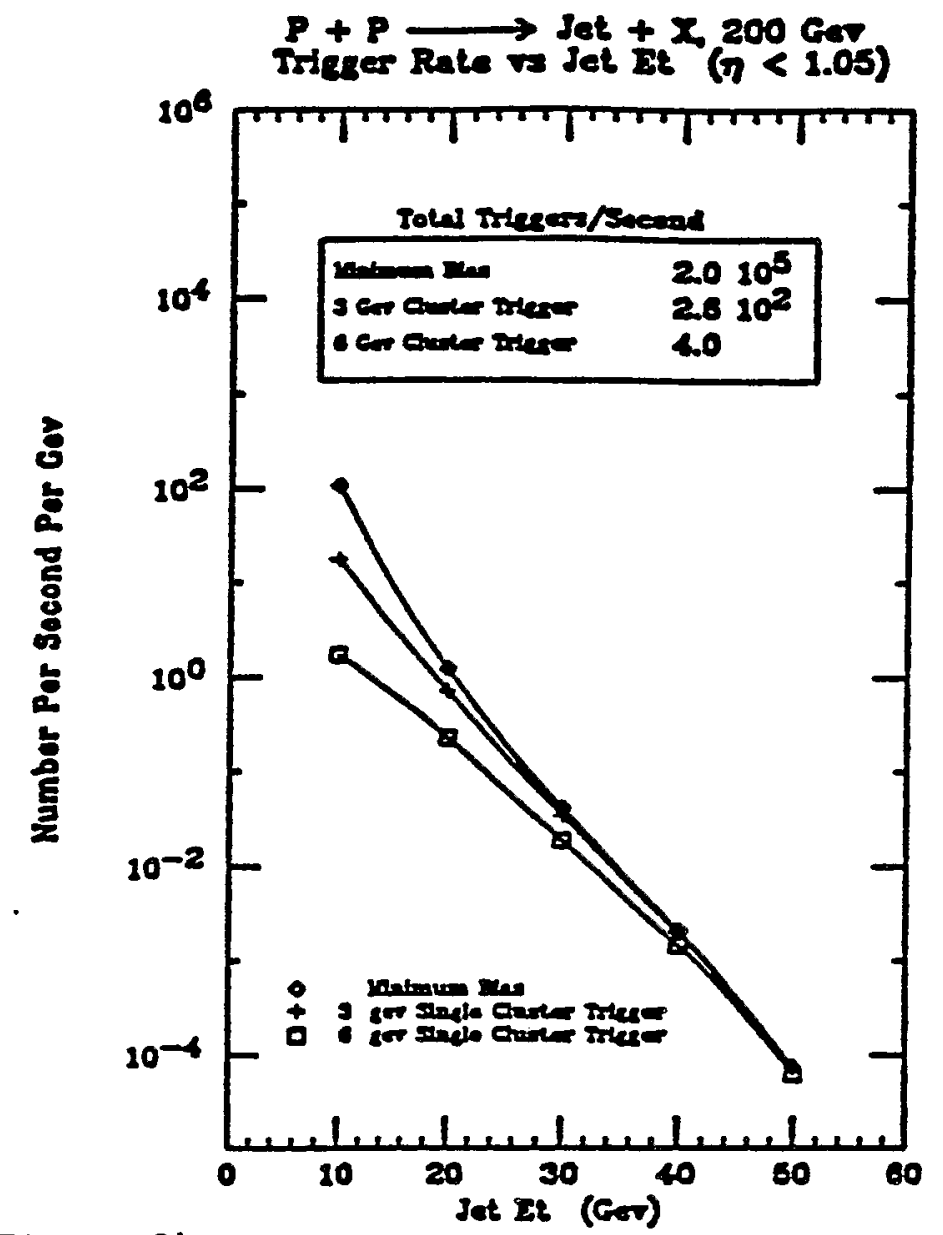

- Figure C4

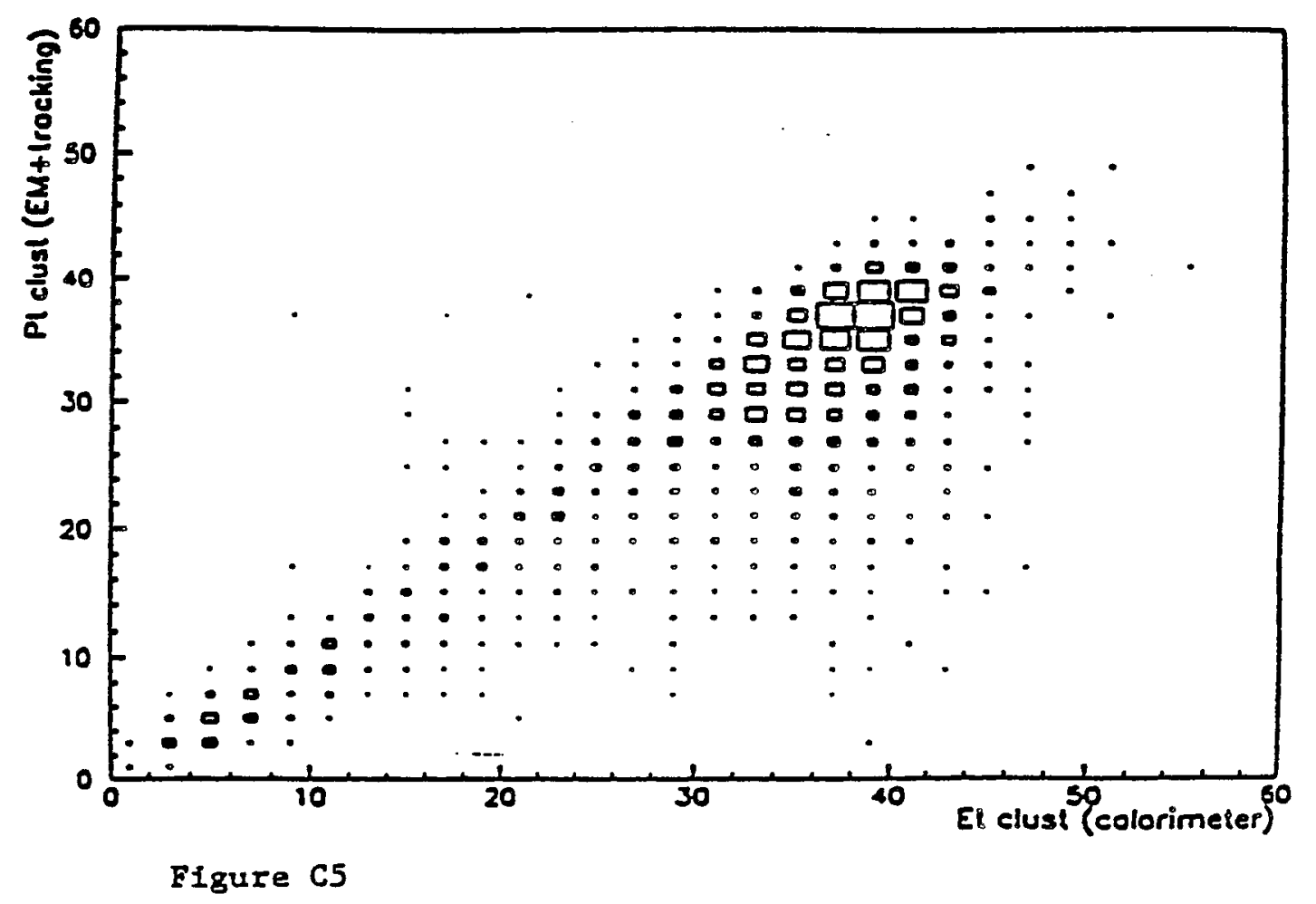




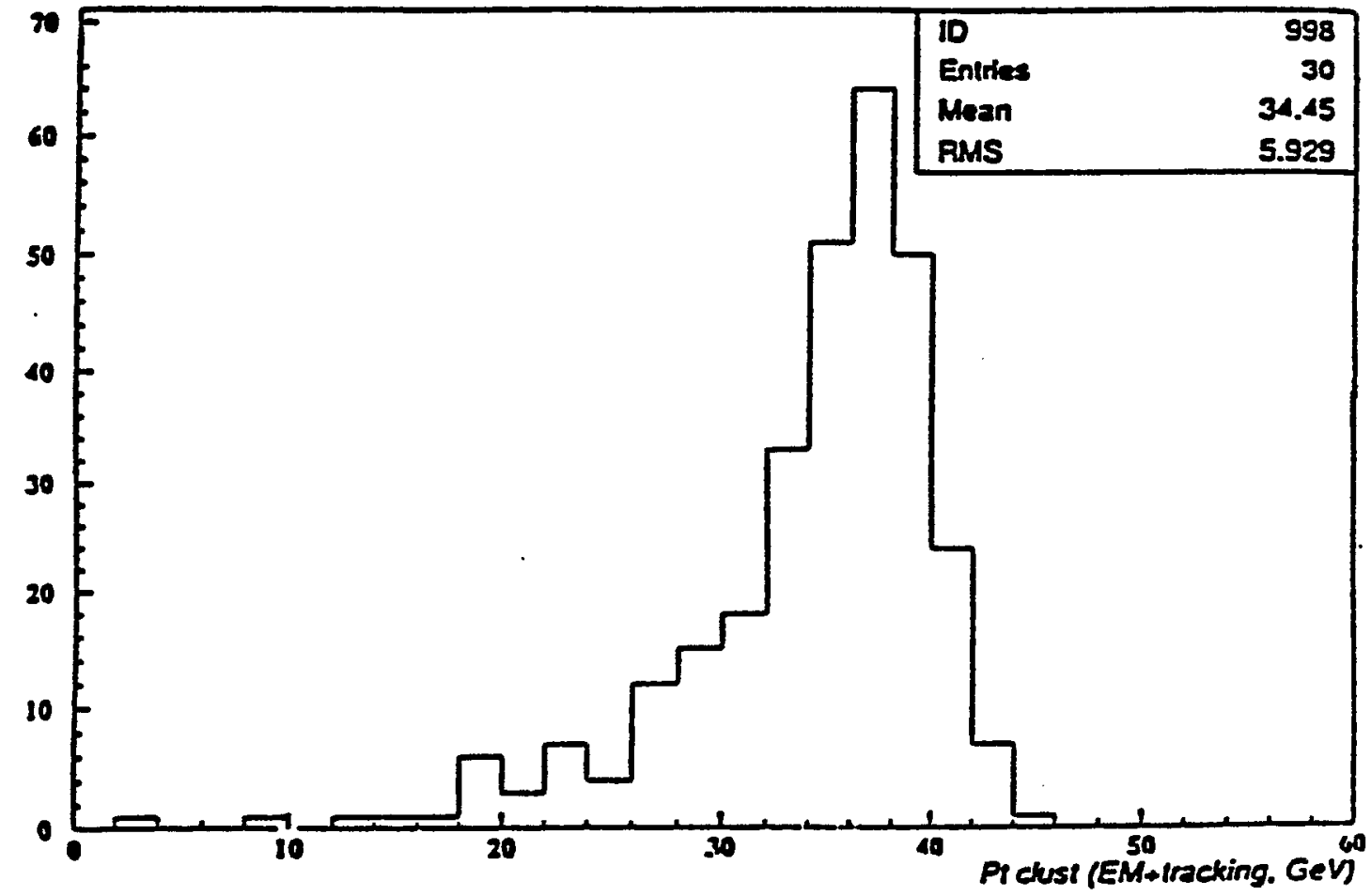

Figure C6 

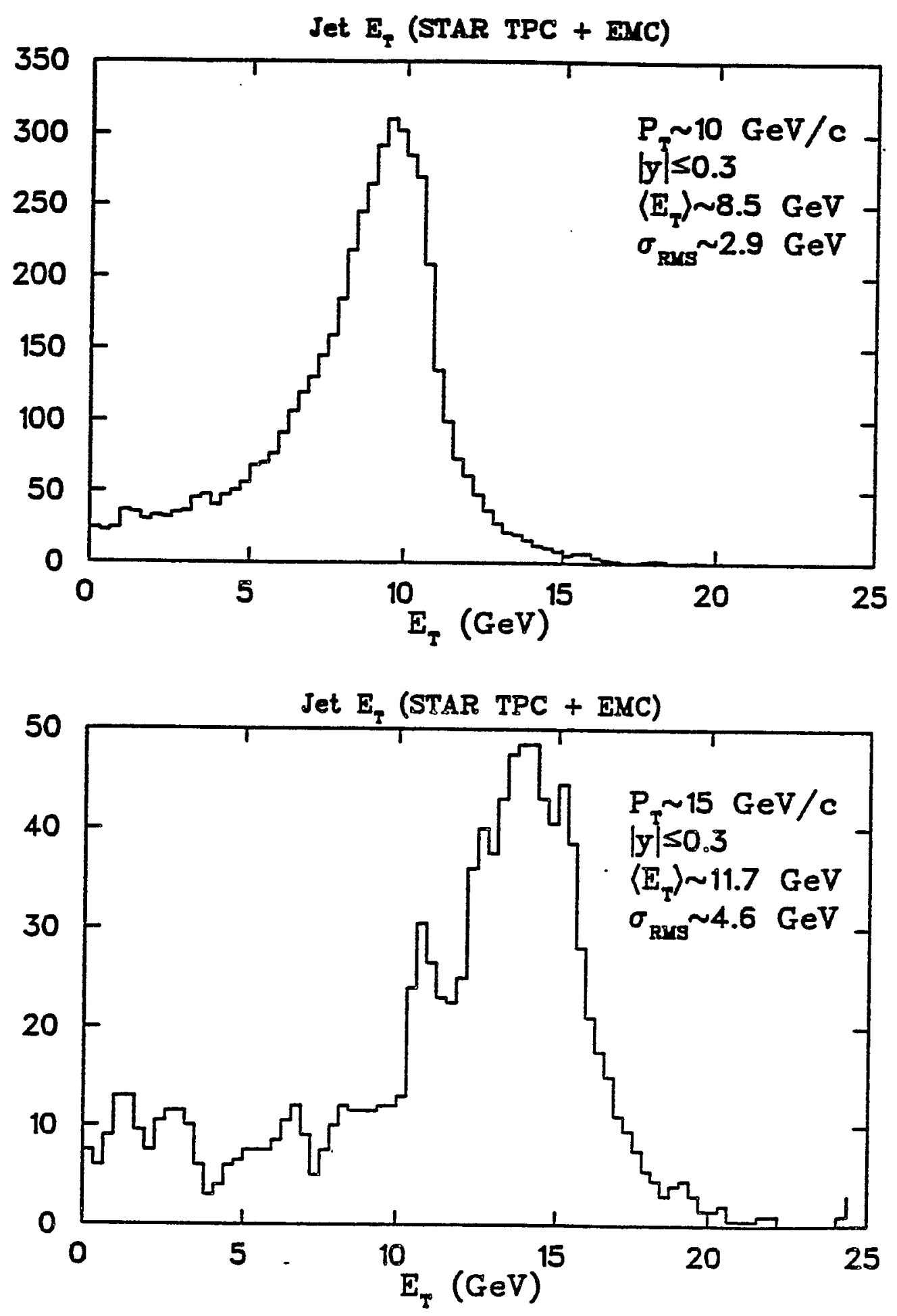

Figure C7 
TPC hat densits - ptp collisions

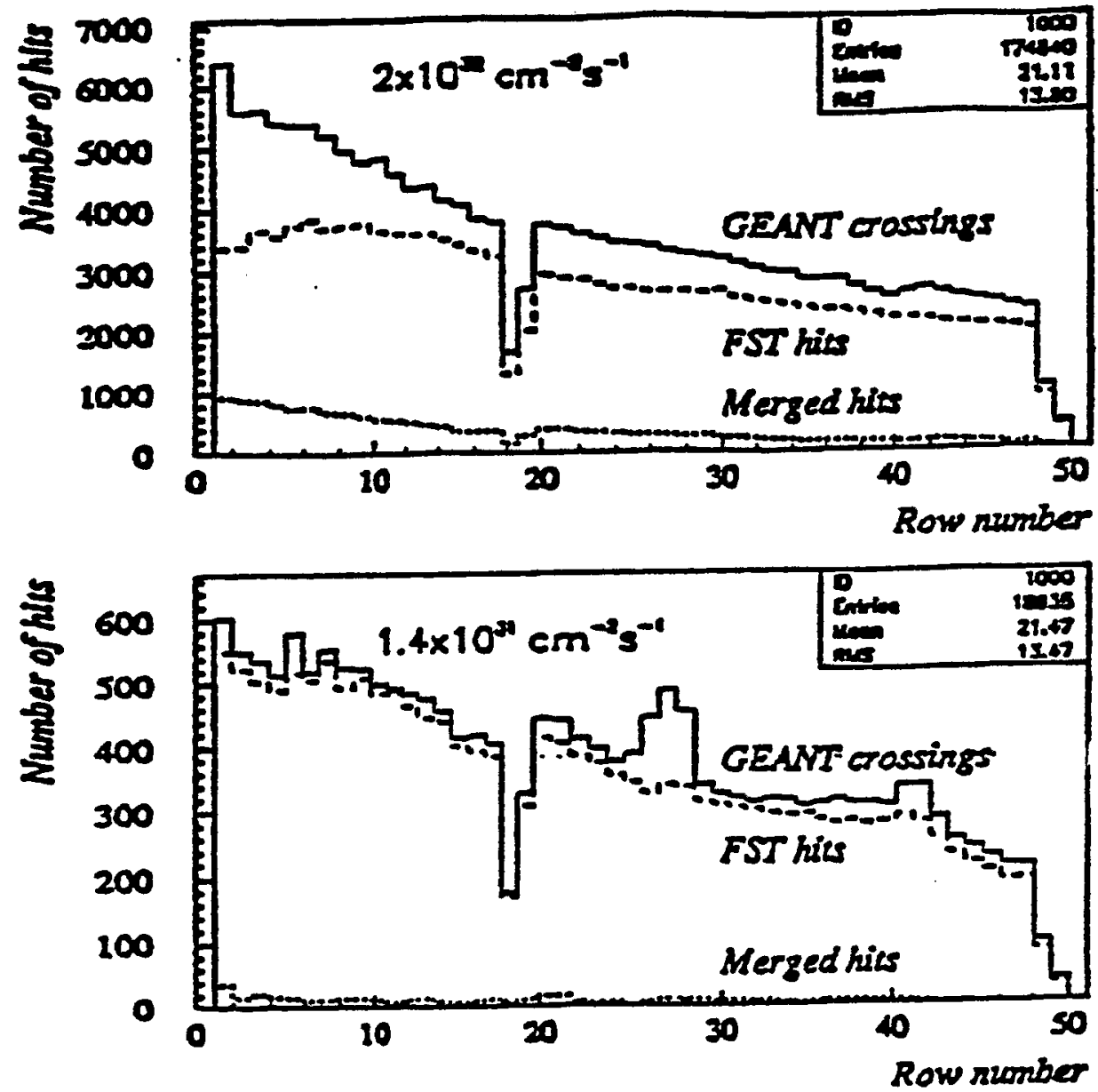

Figure C8

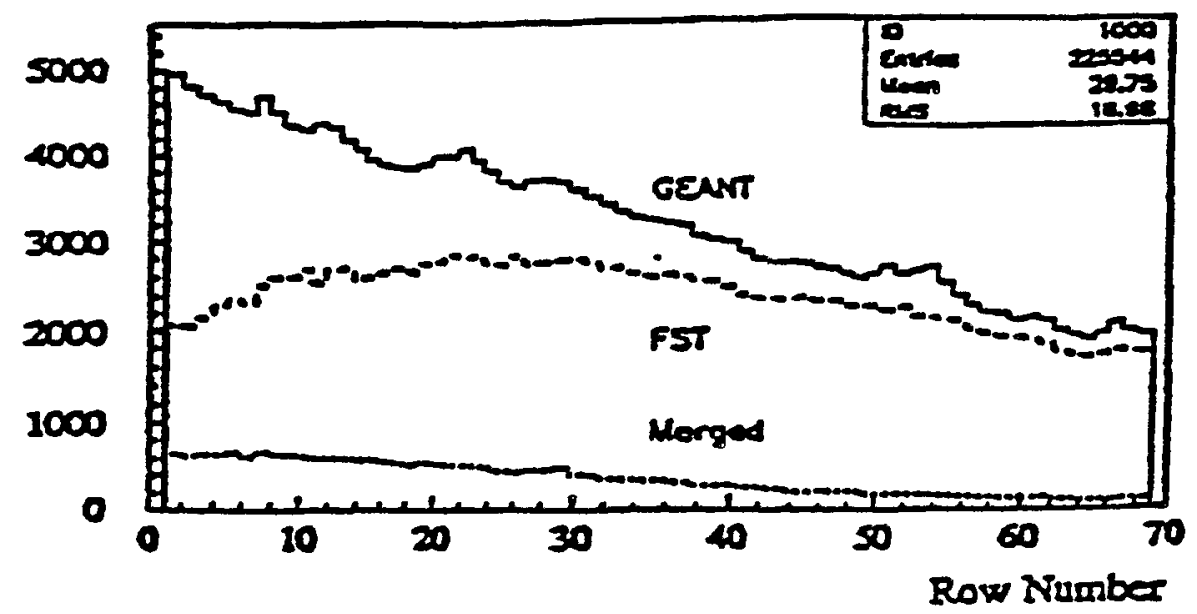

Total hit multiplicity as a function of pad row for a central Au + Au coent.

Figure C9 
Momentum resolution - p+p 1.4x10^31
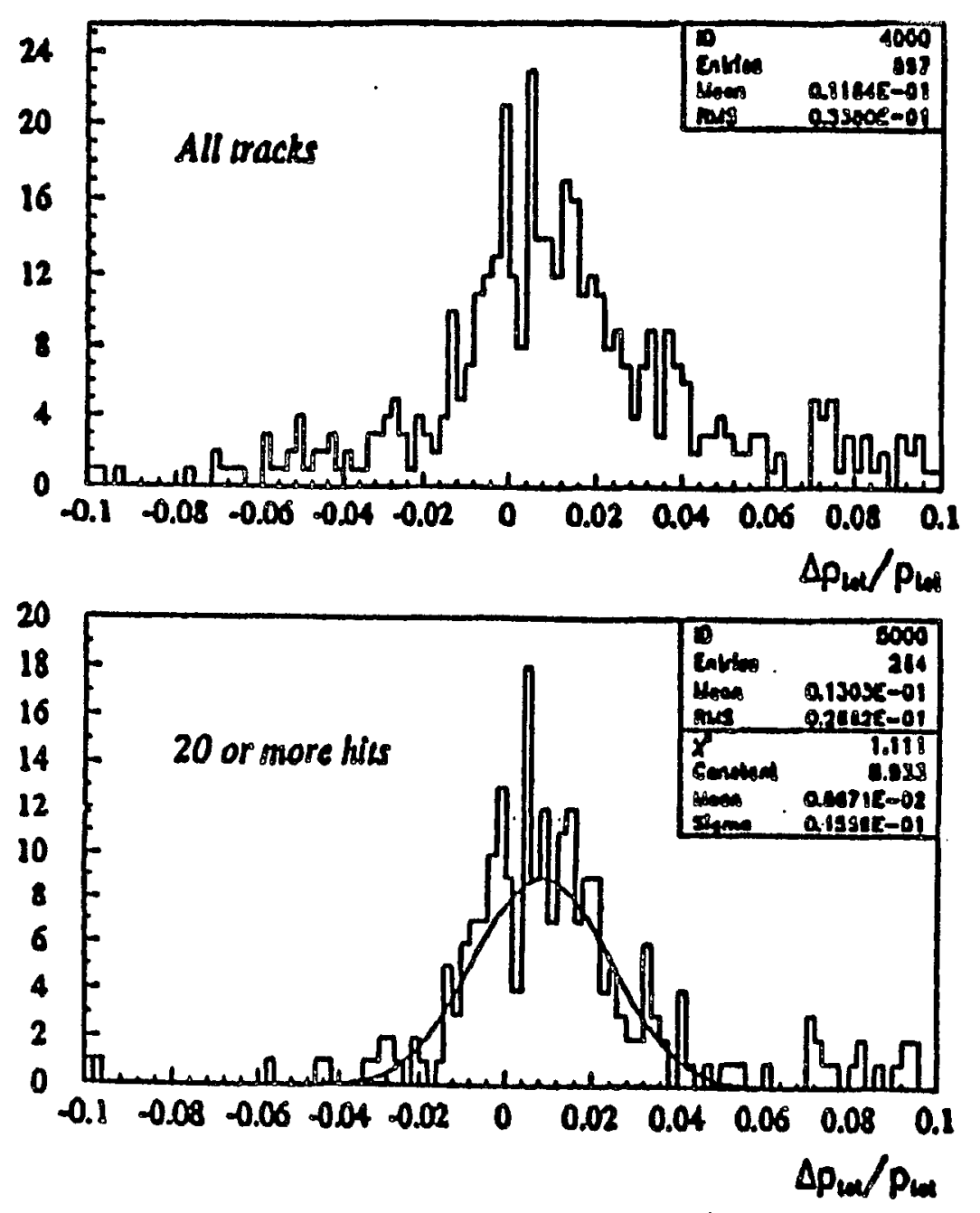

Momonsum resolution - p+p $42 \times 10$ a32
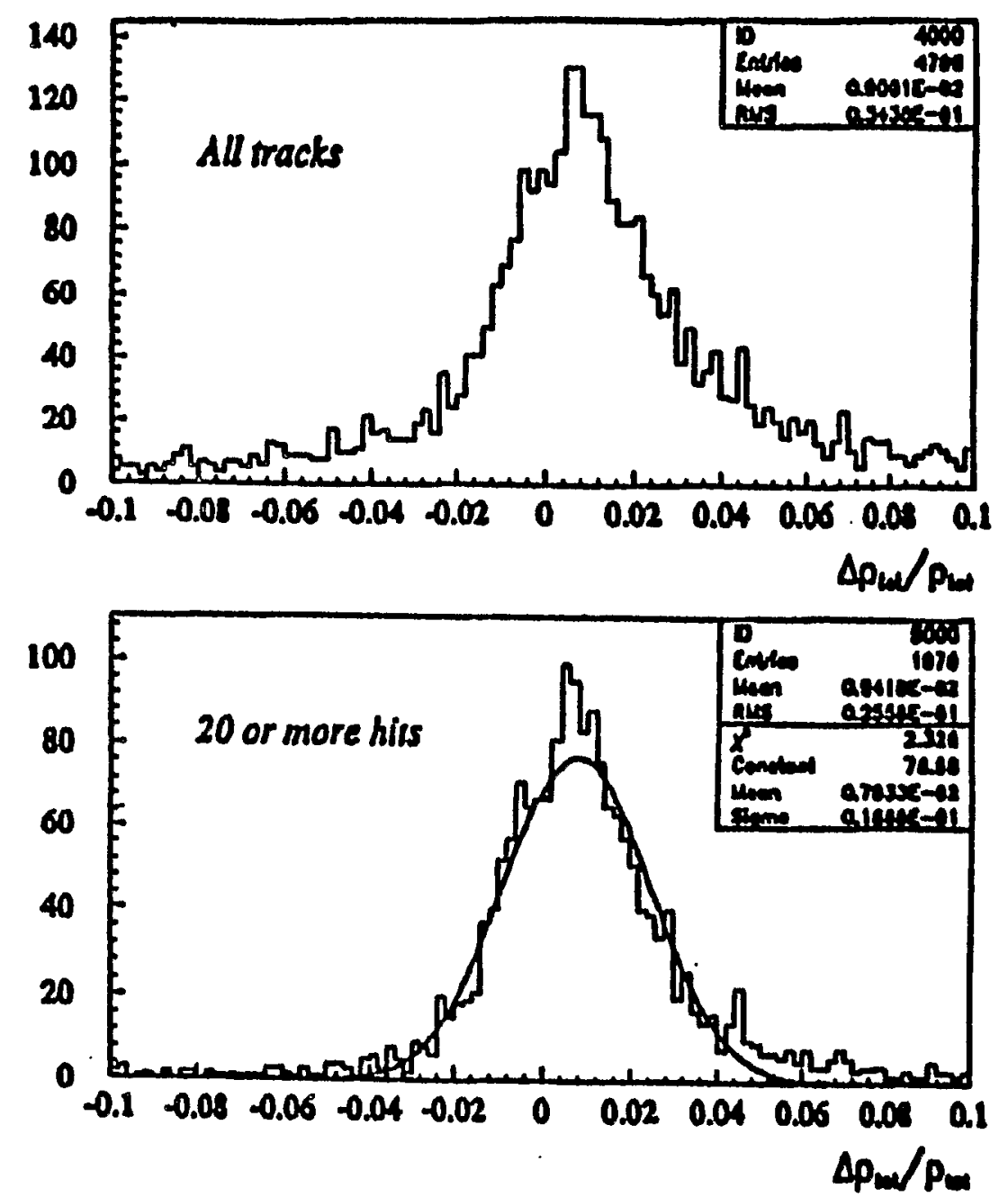

Figure C10 
EVAL: 20 impact parameter - pp @2x10^32
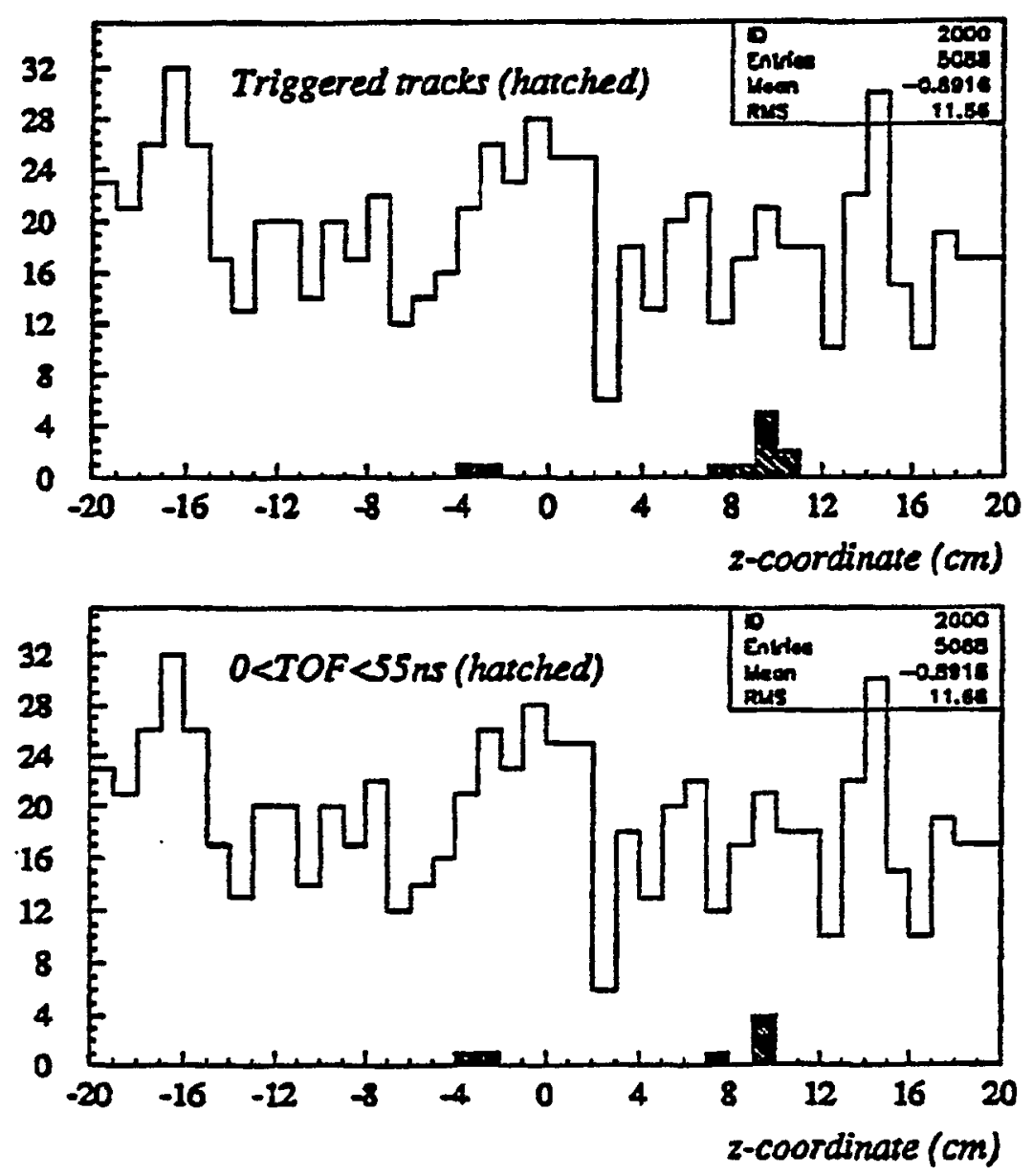

Figure C11 


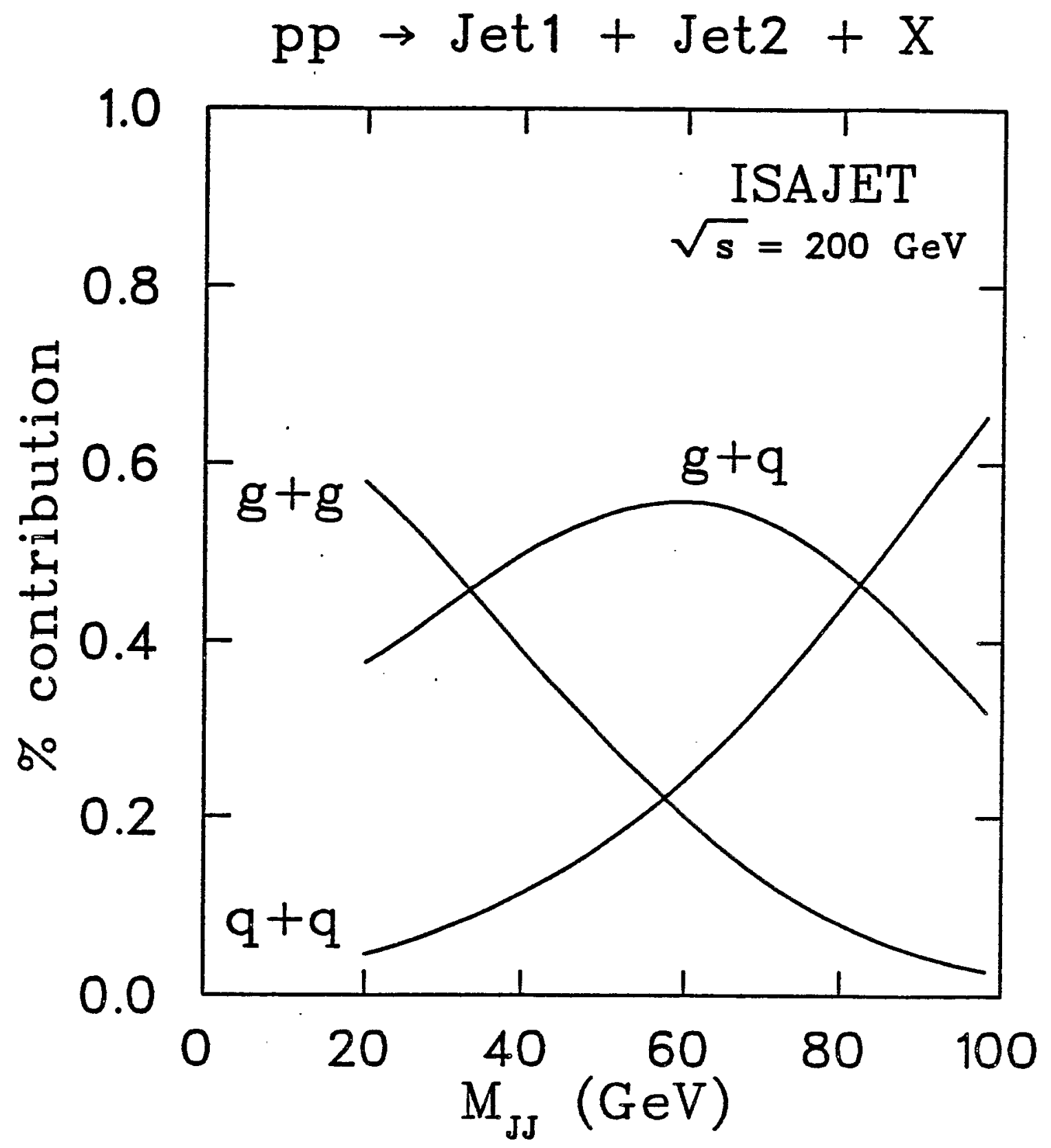

Figure $\mathrm{C} 12$ 
STAR $x_{1}$ and $x_{2}$ coverage for $x_{T}=0.1$

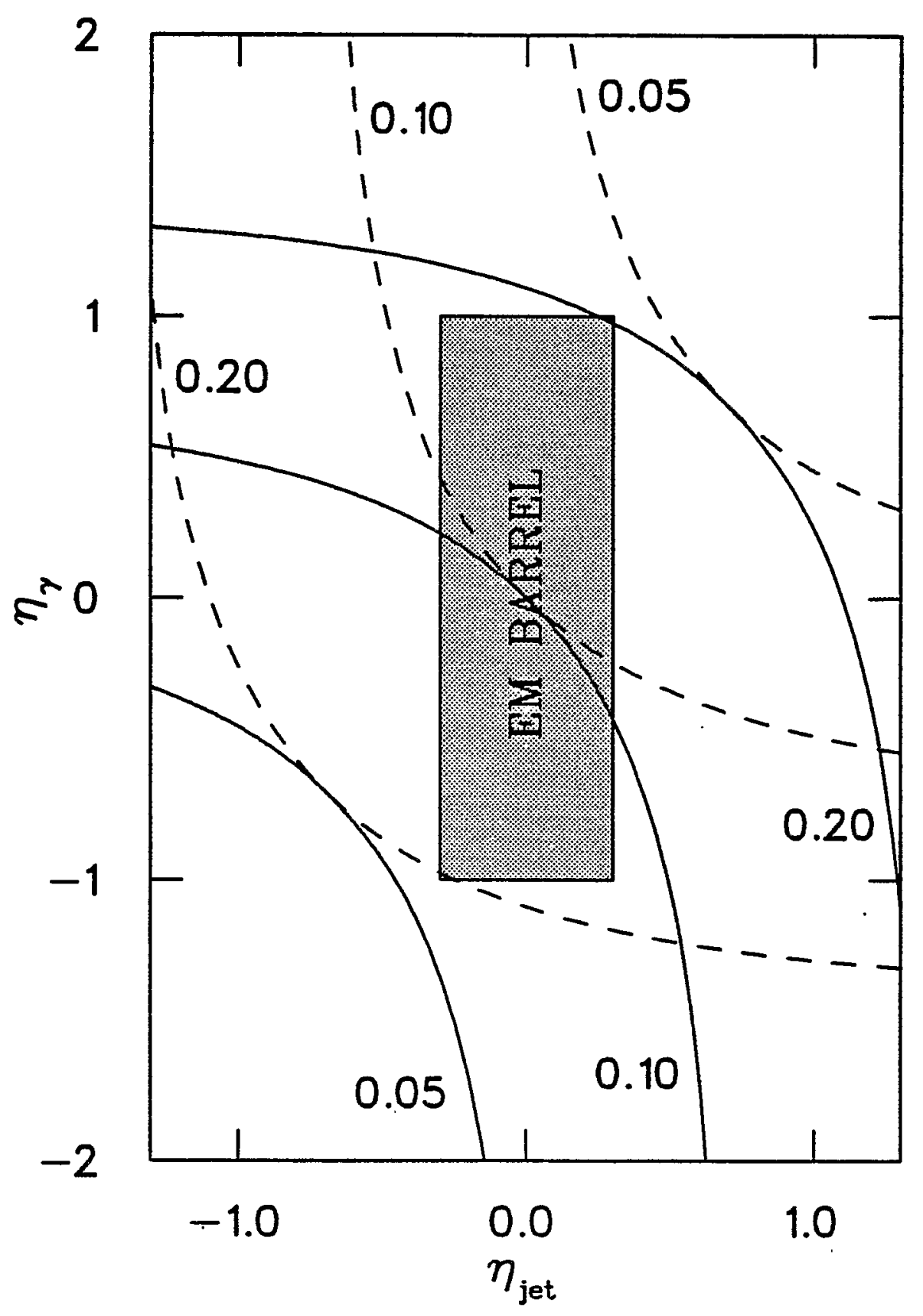

Figure C13 


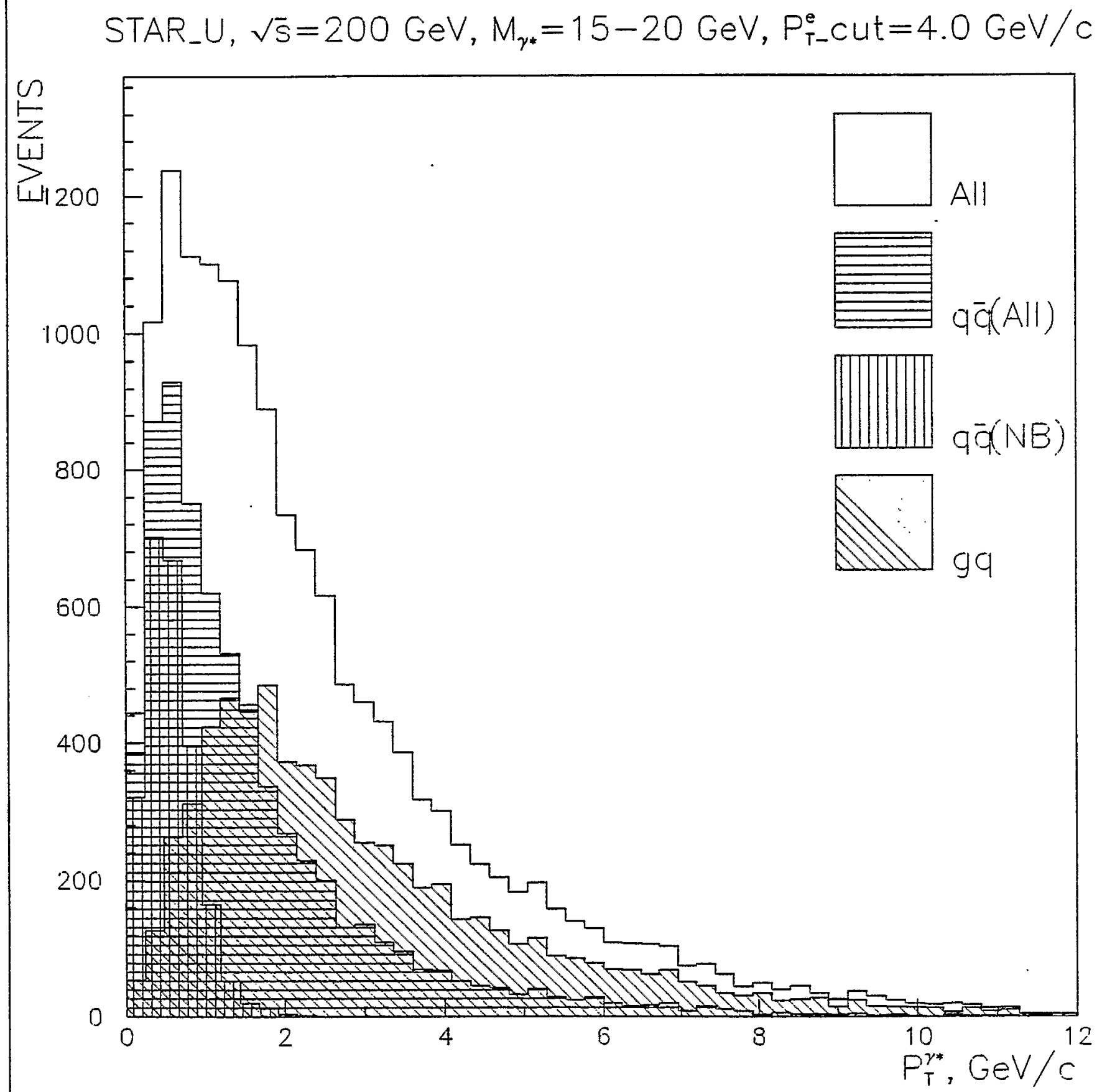

Figure C.14 


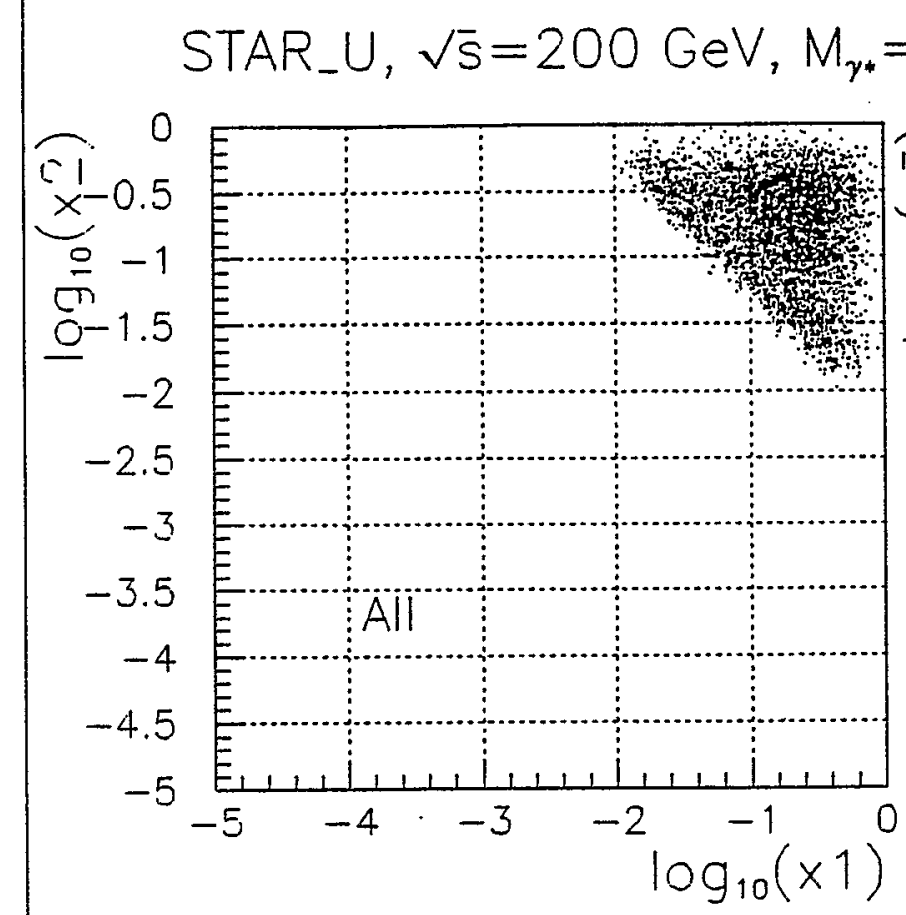

$=15-20 \mathrm{GeV}, P_{\mathrm{T}-}^{\mathrm{e}} \mathrm{cut}=4.0 \mathrm{GeV} / \mathrm{c}$
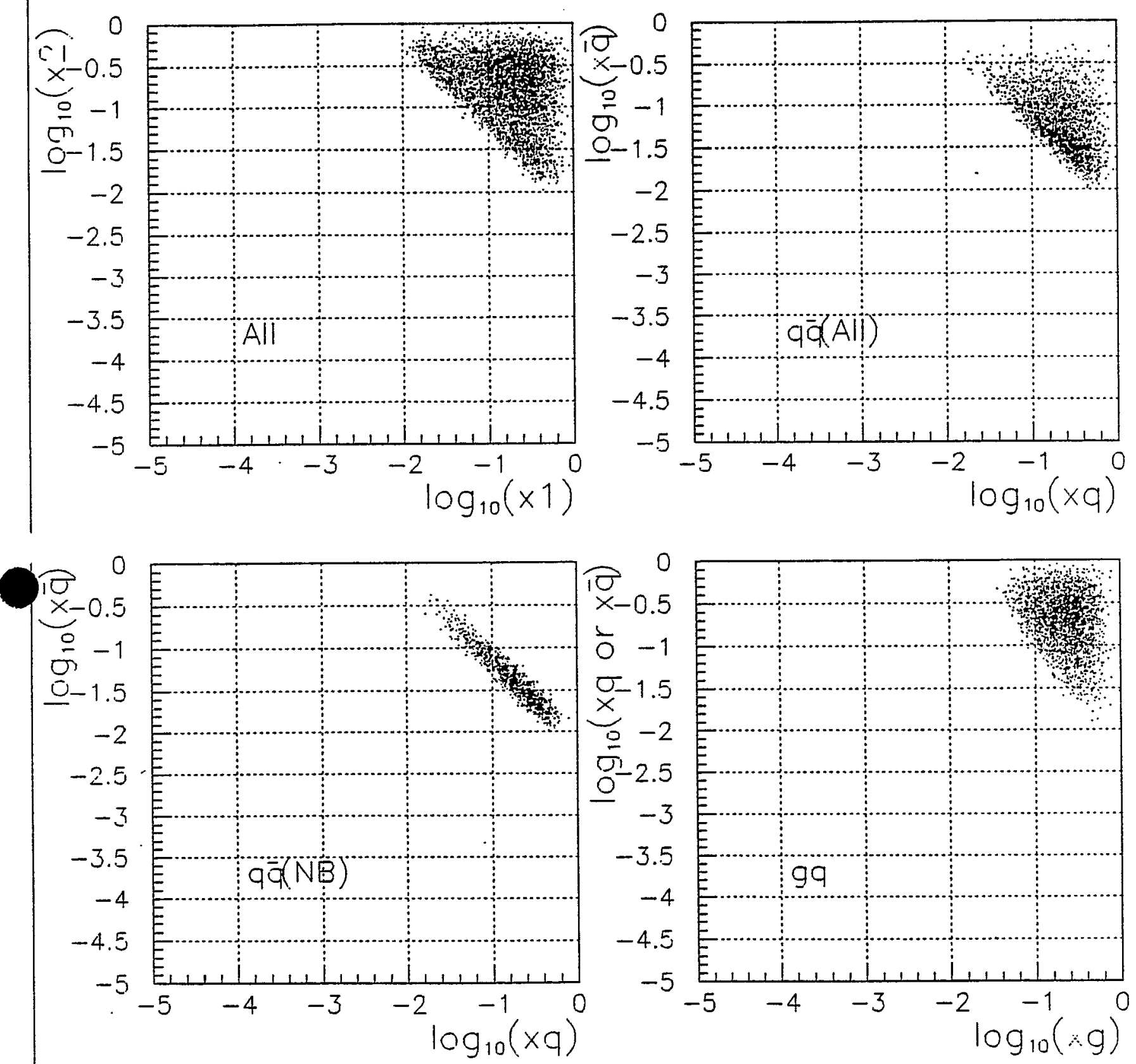

Figure C.15 